\title{
Dipole-LUMO/Dipolarophile-HOMO Controlled Asymmetric Cycloadditions of Carbonyl Ylides Catalyzed by Chiral Lewis Acids
}

\author{
Hiroyuki Suga, * Daisuke Ishimoto, Satoshi Higuchi, Motoo Ohtsuka, Tadashi Arikawa, Teruko \\ Tsuchida, Akikazu Kakehi, and Toshihide Baba
}

Department of Chemistry and Material Engineering, Faculty of Engineering, Shinshu University, Wakasato, Nagano 380-8553, Japan, and Department of Environmental Chemistry \& Engineering, Interdisciplinary Graduate School of Science \& Engineering, Tokyo Institute of Technology, G1-14, 4259 Nagatsuta, Midori-ku, Yokohama 226-8503, Japan

\section{Supporting Information}

Table of Contents

Experimental Section (General Methods, Materials, and General Procedure)...............S2-S16

Influence of Ionic Radius on Diastereo- and Enantioselectivity. S17-S19

The interaction between HOMO and LUMO on the basis of frontier orbital theory. S20

Reaction of epoxy compound $\mathbf{I}$ as a carbonyl ylide precursor. S20-S21

References and Notes.

${ }^{1} \mathrm{H}$ and ${ }^{13} \mathrm{C}$ NMR (DEPT) Spectra of cycloadducts .S23-S53 


\section{Supporting Information}

\section{Experimental Section}

General. Melting points were determined on a Yanaco MP-13 melting point apparatus and are uncorrected. IR spectra were taken with a JASCO FT/IR-5300S spectrophotometer. ${ }^{1} \mathrm{H}$ NMR spectra were recorded on a JEOL JNM-GX400 (400 MHz) spectrometer. Chemical shifts are expressed in parts per million downfield from tetramethylsilane as an internal standard. ${ }^{13} \mathrm{C}$ NMR spectra were recorded on JEOL JNM-GX400 (100 MHz) spectrometer using broadband proton decoupling. Chemical shifts are expressed in parts per million using the middle resonance of $\mathrm{CDCl}_{3}(77.0 \mathrm{ppm})$ as an internal standard. High-resolution mass spectra were obtained on HITACHI M-80B spectrometer. Elemental analyses were performed on a Perkin-Elmer $2400 \mathrm{CHN}$ recorder. High performance liquid chromatography was performed on a SHIMADZU LC-VP system or a JASCO PU-2080/UV-2075 system. Optical rotations were recorded with a JASCO P-1010 polarimeter. For preparative column chromatography, Wakogel C-300HG was employed. Medium-pressure liquid chromatography was carried out using a column packed with Wakogel C-300HG. All reactions were carried out under an argon atmosphere in dried glassware.

Materials. $o$-(Methoxycarbonyl)- $\alpha$-diazoacetophenone (1) was prepared by the procedure in the previous paper. ${ }^{1} \alpha, \alpha^{\prime}$-Dicarbonyl diazo substrates $\mathbf{4}-\mathbf{1 1}^{2}$ and 1-diazo-2,5-hexanedione $(\mathbf{2 0})^{6}$ were prepared according to the procedure reported by Padwa. Ethyl vinyl ether, Butyl vinyl ether, $t$-Butyl vinyl ether, Cyclohexyl vinyl ether, 2,3-dihydrofuran, and $\mathrm{Rh}_{2}(\mathrm{OAc})_{4}$ were commercially available, and used without further purification. Lanthanoid triflates were commercially available, and dried in vacuo at $200{ }^{\circ} \mathrm{C}$ for $12 \mathrm{~h}$ before use. 2,6-Bis(oxazolinyl)pyridines (Pybox) were prepared by the procedure in the literatures. ${ }^{3}$ Powdered $4 \AA$ molecular sieves (MS $4 \AA$ ) was commercially available and dried in vacuo at $200{ }^{\circ} \mathrm{C}$ for $12 \mathrm{~h}$ before use. $\mathrm{Ni}\left(\mathrm{ClO}_{4}\right)_{2} \cdot 6 \mathrm{H}_{2} \mathrm{O}$ and $\mathrm{Ni}\left(\mathrm{BF}_{4}\right)_{2} \cdot 6 \mathrm{H}_{2} \mathrm{O}$ were commercially available, and used without further purification. Chiral Binaphthyldiimine (BINIM) ligands were prepared by the procedure reported previously. ${ }^{4} \mathrm{CH}_{2} \mathrm{Cl}_{2}$ was commercially available, and used without further purification.. 


\section{Supporting Information}

General Procedure for the Reaction of $o$-Methoxycarbonyl- $\alpha$-diazoacetophenone (1) with Vinyl Ethers Was Exemplified the Reaction with Cyclohexyl Vinyl Ether Catalyzed by (4S,5S)Pybox-Ph 2 -Eu(III) Complex: A solution of 2,6-bis[(4S,5S)-4,5-diphenyl-2-oxazolin-2-yl]pyridine $\left((4 S, 5 S)-\right.$ Pybox- $\left.\mathrm{Ph}_{2}, 26.1 \mathrm{mg}, 0.05 \mathrm{mmol}\right)$ in THF $(1.5 \mathrm{~mL})$ was added to a solution of Eu(OTf $)_{3}(29.9$ $\mathrm{mg}, 0.05 \mathrm{mmol})$ in THF $(1 \mathrm{~mL})$. After stirring the mixture for $2 \mathrm{~h}$, the solvent was removed under reduced pressure and resulting solid was dried in vacuo at room temperature for $5 \mathrm{~h}$. A solution of $\mathrm{Eu}(\mathrm{III})-\mathrm{Pybox}$ complex in $\mathrm{CH}_{2} \mathrm{Cl}_{2}(3 \mathrm{~mL})$ was transferred to a two-necked round-bottomed flask (30 $\mathrm{mL})$ equipped with reflux condenser. After added MS $4 \AA$ ( $0.5 \mathrm{~g})$, cyclohexyl vinyl ether $(126 \mathrm{mg}, 1.00$ mmol), $\mathrm{Rh}_{2}(\mathrm{OAc})_{4}(4.4 \mathrm{mg}, \quad 0.01 \mathrm{mmol})$ and $\mathrm{CH}_{2} \mathrm{Cl}_{2}(1 \mathrm{~mL})$, successively, a solution of diazoacetophenone $1(102 \mathrm{mg}, 0.50 \mathrm{mmol})$ in $\mathrm{CH}_{2} \mathrm{Cl}_{2}(5 \mathrm{~mL})$ was added over a period of $1 \mathrm{~h}$ using a syringe pump under reflux (bath temp. $55{ }^{\circ} \mathrm{C}$ ). The syringe was washed with $\mathrm{CH}_{2} \mathrm{Cl}_{2}(1 \mathrm{~mL})$. After removal of MS $4 \AA$ through celite, the reaction mixture was filtered through a plug of silica gel $(3 \mathrm{~cm})$ with AcOEt/hexane $(1: 1,80 \mathrm{~mL})$ as an eluent. The solvent was removed in vacuo, and the residue was purified by column chromatography (99:1 hexane/AcOEt) to provide a quantitative amount (quant) of endo-3d and exo-3d. Relative stereochmistry (endo/exo) of the products could be determined by ${ }^{1} \mathrm{H}$ NMR analysis on the basis of a coupling constant between H-1 and H-7 which reported previously (endo: $7.3 \mathrm{~Hz}$, exo: $0 \mathrm{~Hz}){ }^{7}$ The endolexo ratio was determined by ${ }^{1} \mathrm{H} \mathrm{NMR}$ analysis (endo : exo $=88$ : 12) on the basis of the integration corresponding to one of the methylene protons at 6 position. The enantiomeric excess (endo) was determined by HPLC analysis (DAICEL Chiralpak AD-H, $1: 99 i$ $\mathrm{PrOH} / \mathrm{hexane}$, flow $0.5 \mathrm{~mL} / \mathrm{min}, 35^{\circ} \mathrm{C}$ ) $\mathrm{t}_{\mathrm{R}}=29.4 \mathrm{~min}$ (minor), $71.1 \mathrm{~min}$ (major).

\section{7-endo-Cyclohexyloxy-5-methoxy-8-oxabenzo[c]bicyclo[3.2.1]octan-2-one $\quad$ (endo-3d):}

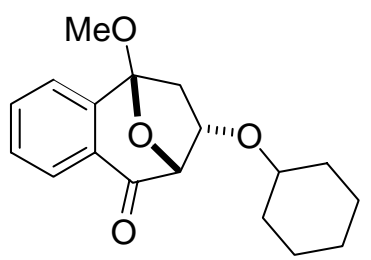

Colorless viscous oil; $[\alpha]_{\mathrm{D}}{ }^{25}=-99.9\left(\right.$ c 1.00, $\left.\mathrm{CHCl}_{3}\right)($ endo $:$ exo $=88: 12$, 95\% ee (endo)); IR (neat, mixture with minor exo-3d): 3021, 2938, 2859, $1707,1603,1453,1300,1263,1215,1161,1101,1078,1053,1026,1007 \mathrm{~cm}^{-}$

${ }^{1} ;{ }^{1} \mathrm{H} \mathrm{NMR}\left(\mathrm{CDCl}_{3}\right) \delta 1.00-1.82(10 \mathrm{H}, \mathrm{m}), 1.99(1 \mathrm{H}, \mathrm{dd}, J=13.2,2.7 \mathrm{~Hz})$, $2.61(1 \mathrm{H}, \mathrm{dd}, J=13.2,9.8 \mathrm{~Hz}), 3.22-3.32(1 \mathrm{H}, \mathrm{m}), 3.50(3 \mathrm{H}, \mathrm{s}), 4.71(1 \mathrm{H}, \mathrm{ddd}, J=2.7,9.8,7.3 \mathrm{~Hz}$,$) ,$ $4.91(1 \mathrm{H}, \mathrm{d}, J=7.3 \mathrm{~Hz}), 7.40-7.49(2 \mathrm{H}, \mathrm{m}), 7.57-7.64(1 \mathrm{H}, \mathrm{m}), 7.98-8.04(1 \mathrm{H}, \mathrm{m}) ;{ }^{13} \mathrm{C} \mathrm{NMR}$ 


\section{Supporting Information}

$\left(\mathrm{CDCl}_{3}\right) \delta 24.0\left(\mathrm{CH}_{2}\right), 24.1\left(\mathrm{CH}_{2}\right), 25.7\left(\mathrm{CH}_{2}\right), 31.7\left(\mathrm{CH}_{2}\right), 32.4\left(\mathrm{CH}_{2}\right), 42.7\left(\mathrm{CH}_{2}\right), 51.8\left(\mathrm{CH}_{3}\right), 73.5$ $(\mathrm{CH}), 77.6(\mathrm{CH}), 84.0(\mathrm{CH}), 106.4(\mathrm{C}), 122.8(\mathrm{CH}), 126.4(\mathrm{CH}), 128.3(\mathrm{CH}), 131.1(\mathrm{C}), 133.5(\mathrm{CH})$, 145.1 (C), 192.8 (C); MS (EI, mixture with minor exo-3d) m/z $302\left(\mathrm{M}^{+}\right), 220,204,176,161,143,133$, $115,103,91,77,67,55,47,37,24,16$. Anal. Calcd for $\mathrm{C}_{18} \mathrm{H}_{22} \mathrm{O}_{4}: \mathrm{C}, 71.50 ; \mathrm{H}, 7.33 \%$. Found: C, 71.58; H, 7.60\% (mixture with minor exo-3d).

\section{7-exo-Cyclohexyloxy-5-methoxy-8-oxabenzo[c]bicyclo[3.2.1]octan-2-one}

(exo-3d):

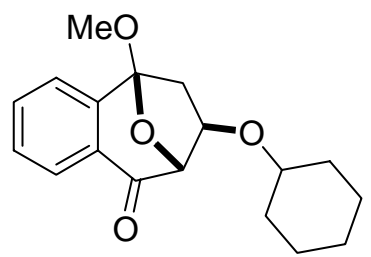

Although exo-3d could not separate by chromatography from a mixture with major endo-3d, it could characterize by ${ }^{1} \mathrm{H}$ and ${ }^{13} \mathrm{C}$ NMR. ${ }^{1} \mathrm{H}$ NMR $\left(\mathrm{CDCl}_{3}\right) \delta$ $1.00-1.82(10 \mathrm{H}, \mathrm{m}), 2.31(1 \mathrm{H}, \mathrm{dd}, J=13.2,3.9 \mathrm{~Hz}), 2.39(1 \mathrm{H}, \mathrm{dd}, J=13.2$, $7.3 \mathrm{~Hz}), 3.32-3.38(1 \mathrm{H}, \mathrm{m}), 3.55(3 \mathrm{H}, \mathrm{s}), 4.14(1 \mathrm{H}, \mathrm{dd}, J=3.9,7.3 \mathrm{~Hz}), 4.74$ $(1 \mathrm{H}, \mathrm{s}), 7.40-7.49(2 \mathrm{H}, \mathrm{m}), 7.57-7.64(1 \mathrm{H}, \mathrm{m}), 7.93-7.98(1 \mathrm{H}, \mathrm{m}) ;{ }^{13} \mathrm{C} \mathrm{NMR}\left(\mathrm{CDCl}_{3}\right) \delta 24.1\left(\mathrm{CH}_{2}\right)$, $24.2\left(\mathrm{CH}_{2}\right), 29.7\left(\mathrm{CH}_{2}\right), 32.0\left(\mathrm{CH}_{2}\right), 32.8\left(\mathrm{CH}_{2}\right), 42.6\left(\mathrm{CH}_{2}\right), 52.0\left(\mathrm{CH}_{3}\right), 76.9(\mathrm{CH}), 77.2(\mathrm{CH}), 87.8$ $(\mathrm{CH}), 107.4(\mathrm{C}), 122.9(\mathrm{CH}), 126.7(\mathrm{CH}), 128.5(\mathrm{CH}), 129.2(\mathrm{C}), 134.2(\mathrm{CH}), 145.5(\mathrm{C}), 193.6(\mathrm{C})$. The enantiomeric excess was determined by HPLC analysis (DAICEL Chiralpak AD-H, $1: 99 i$ PrOH/hexane, flow $0.5 \mathrm{~mL} / \mathrm{min}, 35^{\circ} \mathrm{C}$ ) $\mathrm{t}_{\mathrm{R}}=39.6 \mathrm{~min}$ (minor), $36.4 \mathrm{~min}$ (major).

7-endo-Butoxy-5-methoxy-8-oxabenzo[c]bicyclo[3.2.1]octan-2-one (endo-3a): Colorless

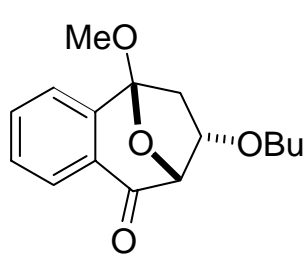

viscous oil; $[\alpha]_{\mathrm{D}}^{25}-77.4\left(\right.$ c $\left.1.00, \mathrm{CHCl}_{3}\right)$ (endo : exo $=81: 19,85 \%$ ee (endo), $62 \%$ ee (exo)), IR (neat, mixture with minor exo-3a) 3031, 3009, 2961, 2874, $1707,1603,1458,1300,1267,1163,1113,1055,1005 \mathrm{~cm}^{-1} ;{ }^{1} \mathrm{H} \mathrm{NMR}\left(\mathrm{CDCl}_{3}\right) \delta$ $0.79(3 \mathrm{H}, \mathrm{t}, J=7.3 \mathrm{~Hz}), 1.15(2 \mathrm{H}$, sext, $J=7.3 \mathrm{~Hz}), 1.35(2 \mathrm{H}$, quint, $J=7.3 \mathrm{~Hz})$, $2.02(1 \mathrm{H}, \mathrm{dd}, J=13.4,2.7 \mathrm{~Hz}), 2.60(1 \mathrm{H}, \mathrm{dd}, J=13.4,9.8 \mathrm{~Hz}), 3.27-3.34(1 \mathrm{H}, \mathrm{m}), 3.45-3.52(1 \mathrm{H}$, m), $3.46(3 \mathrm{H}, \mathrm{s}), 4.53(1 \mathrm{H}, \mathrm{ddd}, J=2.7,9.8,7.1 \mathrm{~Hz}), 4.96(1 \mathrm{H}, \mathrm{d}, J=7.1 \mathrm{~Hz}), 7.42-7.48(2 \mathrm{H}, \mathrm{m}), 7.58$ - $7.64(1 \mathrm{H}, \mathrm{m}), 7.99$ - $8.04(1 \mathrm{H}, \mathrm{m}) ;{ }^{13} \mathrm{C} \mathrm{NMR}\left(\mathrm{CDCl}_{3}\right) \delta 13.9\left(\mathrm{CH}_{3}\right), 19.2\left(\mathrm{CH}_{2}\right), 31.6\left(\mathrm{CH}_{2}\right), 42.0$ $\left(\mathrm{CH}_{2}\right), 51.9\left(\mathrm{CH}_{3}\right), 70.7\left(\mathrm{CH}_{2}\right), 76.6(\mathrm{CH}), 83.8(\mathrm{CH}), 106.4(\mathrm{C}), 122.8(\mathrm{CH}), 126.4(\mathrm{CH}), 128.3(\mathrm{CH})$, $131.0(\mathrm{C}), 133.6(\mathrm{CH}), 145.1$ (C), 192.5 (C); MS (EI, mixture with minor exo-3a) m/z $276\left(\mathrm{M}^{+}\right), 247$, 203, 176, 161, 147, 133, 117, 103, 91, 77, 61, 50, 37, 26, 13; HRMS (EI, mixture with minor exo-3a) 


\section{Supporting Information}

Calcd for $\mathrm{C}_{16} \mathrm{H}_{20} \mathrm{O}_{4}\left(\mathrm{M}^{+}\right)$: 276.1360. Found: 276.1387. Anal. Calcd for $\mathrm{C}_{16} \mathrm{H}_{20} \mathrm{O}_{4}: \mathrm{C}, 69.54 ; \mathrm{H}, 7.30 \%$. Found: C, 69.14; H, 7.70\% (mixture with minor exo-3a). The enantiomeric excess was determined by HPLC analysis (DAICEL Chiralpak AD-H, $1: 99 i$-PrOH/hexane, flow $0.5 \mathrm{~mL} / \mathrm{min}, 35^{\circ} \mathrm{C}$ ) $t_{\mathrm{R}}=22.9$ min (minor), 45.7 min (major). Relative stereochmistry (endo/exo) of the products could be determined by ${ }^{1} \mathrm{H}$ NMR analysis on the basis of a coupling constant between $\mathrm{H}-1$ and $\mathrm{H}-7$ which reported previously (endo: $7.1 \mathrm{~Hz}$, exo: $0 \mathrm{~Hz}){ }^{7}$ The endolexo ratio was determined by ${ }^{1} \mathrm{H}$ NMR analysis (endo : exo $=81: 19)$ on the basis of the integration corresponding to one of the methylene protons at 6 position.

7-exo-Butoxy-5-methoxy-8-oxabenzo[c]bicyclo[3.2.1]octan-2-one (exo-3a): Although exo-

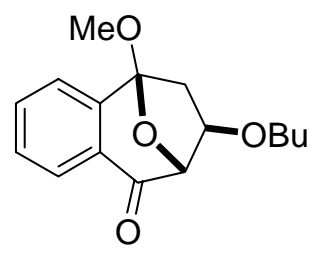

3a could not separate by chromatography from a mixture with major endo-3a, it could characterize by ${ }^{1} \mathrm{H}$ and ${ }^{13} \mathrm{C} \mathrm{NMR} .{ }^{1} \mathrm{H} \mathrm{NMR}\left(\mathrm{CDCl}_{3}\right) \delta 0.93(3 \mathrm{H}, \mathrm{t}, J=7.3$ $\mathrm{Hz}), 1.31-1.45(2 \mathrm{H}, \mathrm{m}), 1.55-1.66(2 \mathrm{H}, \mathrm{m}), 2.31(1 \mathrm{H}, \mathrm{dd}, J=13.2,3.9 \mathrm{~Hz})$, $2.39(1 \mathrm{H}, \mathrm{dd}, J=13.2,7.3 \mathrm{~Hz}), 3.37-3.59(2 \mathrm{H}, \mathrm{m}), 3.55(3 \mathrm{H}, \mathrm{s}), 3.97(1 \mathrm{H}, \mathrm{dd}, J$ $=3.9,7.3 \mathrm{~Hz}), 4.78(1 \mathrm{H}, \mathrm{s}), 7.42-7.48(2 \mathrm{H}, \mathrm{m}), 7.58-7.64(1 \mathrm{H}, \mathrm{m}), 7.93-7.97(1 \mathrm{H}, \mathrm{m}) ;{ }^{13} \mathrm{C} \mathrm{NMR}$ $\left(\mathrm{CDCl}_{3}\right) \delta 14.0\left(\mathrm{CH}_{3}\right), 19.4\left(\mathrm{CH}_{2}\right), 29.7\left(\mathrm{CH}_{2}\right), 31.7\left(\mathrm{CH}_{2}\right), 51.9\left(\mathrm{CH}_{3}\right), 69.7\left(\mathrm{CH}_{2}\right), 79.8(\mathrm{CH}), 86.5$ $(\mathrm{CH}), 107.4(\mathrm{C}), 122.8(\mathrm{CH}), 126.7(\mathrm{CH}), 128.5(\mathrm{CH}), 129.2(\mathrm{C}), 134.3(\mathrm{CH}), 145.6(\mathrm{C}), 193.5(\mathrm{C})$. The enantiomeric excess was determined by HPLC analysis (DAICEL Chiralpak AD-H, $1: 99 i$ PrOH/hexane, flow $0.5 \mathrm{~mL} / \mathrm{min}, 35^{\circ} \mathrm{C}$ ) $\mathrm{t}_{\mathrm{R}}=39.6 \mathrm{~min}$ (minor), $36.4 \mathrm{~min}$ (major).

7-endo-Ethoxy-5-methoxy-8-oxabenzo[c]bicyclo[3.2.1]octan-2-one (endo-3b): Colorless<smiles>CCO[C@H]1CC2(OC)OC(C(=O)c3ccccc32)[C@@H]1OC</smiles>
viscous oil; $[\alpha]_{\mathrm{D}}{ }^{25}-95.1\left(c 1.00, \mathrm{CHCl}_{3}\right)$ (endo : exo $=83: 17,83 \%$ ee (endo), $67 \%$ ee (exo)); IR (neat, mixture with minor exo-3b) 3012, 2980, 1707, 1603, 1458, 1445 , $1300,1267,1252,1215,1165,1115,1053,1005 \mathrm{~cm}^{-1} ;{ }^{1} \mathrm{H} \mathrm{NMR}\left(\mathrm{CDCl}_{3}\right) \delta 1.02(3 \mathrm{H}$, t, $J=7.1 \mathrm{~Hz}), 2.02(1 \mathrm{H}, \mathrm{dd}, J=13.2,2.7 \mathrm{~Hz}), 2.63(1 \mathrm{H}, \mathrm{dd}, J=13.2,10.0 \mathrm{~Hz}), 3.36$ $-3.58(2 \mathrm{H}, \mathrm{m}), 3.47(3 \mathrm{H}, \mathrm{s}), 4.55(1 \mathrm{H}, \mathrm{ddd}, J=2.7,10.0,7.3 \mathrm{~Hz}), 4.97(1 \mathrm{H}, \mathrm{d}, J=7.3 \mathrm{~Hz}), 7.42-7.49$ $(2 \mathrm{H}, \mathrm{m}), 7.58-7.64(1 \mathrm{H}, \mathrm{m}), 7.99-8.06(1 \mathrm{H}, \mathrm{m}) ;{ }^{13} \mathrm{C} \mathrm{NMR}\left(\mathrm{CDCl}_{3}\right) \delta 15.0\left(\mathrm{CH}_{3}\right), 42.1\left(\mathrm{CH}_{2}\right), 51.9$ $\left(\mathrm{CH}_{3}\right), 66.3\left(\mathrm{CH}_{2}\right), 76.4(\mathrm{CH}), 83.8(\mathrm{CH}), 106.4(\mathrm{C}), 122.8(\mathrm{CH}), 126.6(\mathrm{CH}), 128.4(\mathrm{CH}), 130.9(\mathrm{C})$, $133.7(\mathrm{CH}), 145.2(\mathrm{C}), 192.7$ (C); MS (EI) m/z $248\left(\mathrm{M}^{+}\right), 216,203,176,161,147,133,129,115,103$, 


\section{Supporting Information}

89, 76, 61, 47, 39, 26, 13; HRMS (EI) Calcd for $\mathrm{C}_{14} \mathrm{H}_{16} \mathrm{O}_{4}\left(\mathrm{M}^{+}\right)$: 248.1048. Found: 248.1050. Anal. Calcd for $\mathrm{C}_{14} \mathrm{H}_{16} \mathrm{O}_{4}$ : C, 67.73; H, 6.50\%. Found: $\mathrm{C}, 67.30 ; \mathrm{H}, 6.62 \%$. The enantiomeric excess was determined by HPLC analysis (DAICEL Chiralpak AD-H, $1: 99 i$-PrOH/hexane, flow $0.5 \mathrm{~mL} / \mathrm{min}$, $35^{\circ} \mathrm{C}$ ) $\mathrm{t}_{\mathrm{R}}=23.5 \mathrm{~min}$ (minor), $46.9 \mathrm{~min}$ (major). Relative stereochmistry (endolexo) of the products could be determined by ${ }^{1} \mathrm{H}$ NMR analysis on the basis of a coupling constant between $\mathrm{H}-1$ and H-7 which reported previously (endo: $7.3 \mathrm{~Hz}$, exo: $0 \mathrm{~Hz}$ ). ${ }^{7}$ The endolexo ratio was determined by ${ }^{1} \mathrm{H}$ NMR analysis (endo : exo $=83: 17$ ) on the basis of the integration corresponding to one of the methylene protons at 6 position.

7-exo-Ethoxy-5-methoxy-8-oxabenzo[c]bicyclo[3.2.1]octan-2-one (exo-3b): Although exo-<smiles>CCOC1CC2OC(OC)(C1)c1ccccc1C2=O</smiles3b could not separate by chromatography from a mixture with major endo-3a, it could characterize by ${ }^{1} \mathrm{H}$ and ${ }^{13} \mathrm{C}$ NMR. ${ }^{1} \mathrm{H}$ NMR $\left(\mathrm{CDCl}_{3}\right) \delta 1.25(3 \mathrm{H}, \mathrm{t}, J=7.1$ $\mathrm{Hz}), 2.32(1 \mathrm{H}, \mathrm{dd}, J=13.2,3.7 \mathrm{~Hz}), 2.41(1 \mathrm{H}, \mathrm{dd}, J=13.2,7.3 \mathrm{~Hz}), 3.35-3.60$ (2H, m), $3.98(1 \mathrm{H}, \mathrm{dd}, J=3.7,7.3 \mathrm{~Hz}), 4.79(1 \mathrm{H}, \mathrm{s}), 7.42-7.49(2 \mathrm{H}, \mathrm{m}), 7.58-$ $7.64(1 \mathrm{H}, \mathrm{m}), 7.93-7.98(1 \mathrm{H}, \mathrm{m}) ;{ }^{13} \mathrm{C} \mathrm{NMR}\left(\mathrm{CDCl}_{3}\right) \delta 15.3\left(\mathrm{CH}_{3}\right), 42.2\left(\mathrm{CH}_{2}\right), 52.0\left(\mathrm{CH}_{3}\right), 65.4\left(\mathrm{CH}_{2}\right)$, $79.7(\mathrm{CH}), 86.6(\mathrm{CH}), 107.4(\mathrm{C}), 122.9(\mathrm{CH}), 126.8(\mathrm{CH}), 128.6(\mathrm{CH}), 129.3(\mathrm{C}), 134.3(\mathrm{CH}), 145.6(\mathrm{C})$, 193.5 (C). The enantiomeric excess was determined by HPLC analysis (DAICEL Chiralpak AD-H, 1 : $99 \mathrm{i}-\mathrm{PrOH} /$ hexane, flow $0.5 \mathrm{~mL} / \mathrm{min}, 35^{\circ} \mathrm{C}$ ) $\mathrm{t}_{\mathrm{R}}=32.3 \mathrm{~min}$ (major), $42.6 \mathrm{~min}$ (minor).

7-endo-t-Butoxy-5-methoxy-8-oxabenzo[c]bicyclo[3.2.1]octan-2-one (endo-3c): Colorless<smiles>COC1CC2(OC)OC1C(=O)c1ccccc12</smiles>
viscous oil; $[\alpha]_{\mathrm{D}}^{25}-112.8\left(\right.$ c $\left.1.00, \mathrm{CHCl}_{3}\right)$ (endo : exo $=87: 13(88 \%$ ee (endo), 74\% ee (exo)); IR (neat, mixture with minor exo-3c) 3021, 2978, 1709, 1603, $1458,1393,1368,1298,1267,1215,1150,1078,1051,1028,1007 \mathrm{~cm}^{-1} ;{ }^{1} \mathrm{H}$ $\operatorname{NMR}\left(\mathrm{CDCl}_{3}\right) \delta 1.09(9 \mathrm{H}, \mathrm{s}), 1.92(1 \mathrm{H}, \mathrm{dd}, J=13.2,2.7 \mathrm{~Hz}), 2.61(1 \mathrm{H}, \mathrm{dd}, J=$ 13.2, $9.8 \mathrm{~Hz}), 3.46(3 \mathrm{H}, \mathrm{s}), 4.69(1 \mathrm{H}, \mathrm{ddd}, J=2.7,9.8,7.6 \mathrm{~Hz}), 4.77(1 \mathrm{H}, \mathrm{d}, J=7.6 \mathrm{~Hz}), 7.36-7.51$ $(2 \mathrm{H}, \mathrm{m}), 7.50-7.67(1 \mathrm{H}, \mathrm{m}), 7.98-8.04(1 \mathrm{H}, \mathrm{m}) ;{ }^{13} \mathrm{C} \mathrm{NMR}\left(\mathrm{CDCl}_{3}\right) \delta 28.0\left(\mathrm{CH}_{3} \mathrm{x} 3\right), 44.1\left(\mathrm{CH}_{2}\right)$, $51.8\left(\mathrm{CH}_{3}\right), 69.2(\mathrm{CH}), 74.5(\mathrm{C}), 84.9(\mathrm{CH}), 106.5(\mathrm{C}), 122.8(\mathrm{CH}), 126.5(\mathrm{CH}), 128.2(\mathrm{CH}), 131.2(\mathrm{C})$, $133.4(\mathrm{CH}), 145.2(\mathrm{C}), 192.6(\mathrm{C})$; MS (EI) m/z $276\left(\mathrm{M}^{+}\right)$, 261, 221, 203, 179, 160, 145, 129, 115, 103, 


\section{Supporting Information}

91, 76, 57, 49, 39, 26, 13. Anal. Calcd for $\mathrm{C}_{16} \mathrm{H}_{20} \mathrm{O}_{4}$ : C, 69.54; H, 7.30\%. Found: C, 69.44; H, 7.38\%. The enantiomeric excess was determined by HPLC analysis (DAICEL Chiralpak AD-H, $1: 99 i$ $\mathrm{PrOH} /$ hexane, flow $0.5 \mathrm{~mL} / \mathrm{min}, 35^{\circ} \mathrm{C}$ ) $\mathrm{t}_{\mathrm{R}}=27.5 \mathrm{~min}$ (minor), $64.1 \mathrm{~min}$ (major). Relative stereochmistry (endo/exo) of the products could be determined by ${ }^{1} \mathrm{H}$ NMR analysis on the basis of a coupling constant between $\mathrm{H}-1$ and $\mathrm{H}-7$ which reported previously (endo: $7.6 \mathrm{~Hz}$, exo: $0 \mathrm{~Hz}$ ). ${ }^{7}$ The endolexo ratio was determined by ${ }^{1} \mathrm{H}$ NMR analysis (endo : exo $=87: 13$ ) on the basis of the integration corresponding to one of the methylene protons at 6 position.

7-exo-t-Butoxy-5-methoxy-8-oxabenzo[c]bicyclo[3.2.1]octan-2-one (exo-3c): Although exo-

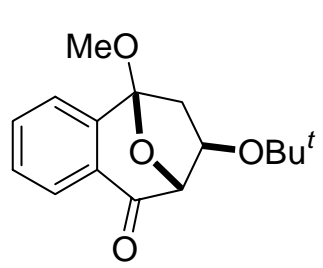

3c could not separate by chromatography from a mixture with major endo-3a, it could characterize by ${ }^{1} \mathrm{H}$ and ${ }^{13} \mathrm{C}$ NMR. ${ }^{1} \mathrm{H}$ NMR $\left(\mathrm{CDCl}_{3}\right) \delta 1.21(9 \mathrm{H}, \mathrm{s}), 2.28$ $(1 \mathrm{H}, \mathrm{dd}, J=13.2,4.2 \mathrm{~Hz}), 2.39(1 \mathrm{H}, \mathrm{dd}, J=13.2,7.6 \mathrm{~Hz}), 3.54(3 \mathrm{H}, \mathrm{s}), 4.18(1 \mathrm{H}$, $\mathrm{dd}, J=4.2,7.6 \mathrm{~Hz}) 4.67(1 \mathrm{H}, \mathrm{s}), 7.36-7.51(2 \mathrm{H}, \mathrm{m}), 7.50-7.67(1 \mathrm{H}, \mathrm{m}), 7.94-$

$7.98(1 \mathrm{H}, \mathrm{m}) ;{ }^{13} \mathrm{C} \mathrm{NMR}\left(\mathrm{CDCl}_{3}\right) \delta 28.3\left(\mathrm{CH}_{3} \mathrm{x} 3\right), 44.1\left(\mathrm{CH}_{2}\right), 52.1\left(\mathrm{CH}_{3}\right), 72.5(\mathrm{CH}), 74.9(\mathrm{C}), 89.7$ $(\mathrm{CH}), 107.5(\mathrm{C}), 123.0(\mathrm{CH}), 126.8(\mathrm{CH}), 128.5(\mathrm{CH}), 129.3(\mathrm{C}), 134.2(\mathrm{CH}), 145.5(\mathrm{C}), 193.5(\mathrm{C})$. The enantiomeric excess was determined by HPLC analysis (DAICEL Chiralpak AD-H, $1: 99 i$ $\mathrm{PrOH} / \mathrm{h}$ exane, flow $0.5 \mathrm{~mL} / \mathrm{min}, 35^{\circ} \mathrm{C}$ ) $\mathrm{t}_{\mathrm{R}}=22.5 \mathrm{~min}$ (major), $36.2 \mathrm{~min}$ (minor).

General Procedure for the Reactions of $\alpha, \alpha$ 'Dicarbonyl Diazo Compounds with Vinyl Ethers Was Exemplified the Reaction of Methyl 2-(2-Diazo-1,3-dioxohexyl)benzoate (4) with Butyl Vinyl Ether Catalyzed by $(R)$-BINIM-4Me-2QN-Ni(II) Complex: A solution of $(R)$-BINIM-4Me2QN (29.5 mg, $0.05 \mathrm{mmol})$ in $\mathrm{CH}_{2} \mathrm{Cl}_{2}(2.5 \mathrm{~mL})$ was added to a mixture of powdered MS $4 \AA$ (500 mg) and $\mathrm{Ni}\left(\mathrm{ClO}_{4}\right)_{2} \cdot 6 \mathrm{H}_{2} \mathrm{O}(18.3 \mathrm{mg}, 0.05 \mathrm{mmol})$ in a two-necked round-bottomed flask $(30 \mathrm{~mL})$ equipped with reflux condenser, and then stirred for $6 \mathrm{~h}$ at room temperature. After added butyl vinyl ether (100 $\mathrm{mg}, 1.00 \mathrm{mmol}), \mathrm{Rh}_{2}(\mathrm{OAc})_{4}(4.4 \mathrm{mg}, 0.01 \mathrm{mmol})$ and $\mathrm{CH}_{2} \mathrm{Cl}_{2}(1.5 \mathrm{~mL})$, successively, a solution of diazo compound $4(137 \mathrm{mg}, 0.50 \mathrm{mmol})$ in $\mathrm{CH}_{2} \mathrm{Cl}_{2}(5 \mathrm{~mL})$ was added over a period of $1 \mathrm{~h}$ using a syringe pump under reflux (bath temp. $55{ }^{\circ} \mathrm{C}$ ). The syringe was washed with $\mathrm{CH}_{2} \mathrm{Cl}_{2}(1 \mathrm{~mL})$. After removal of MS $4 \AA$ through celite, the reaction mixture was filtered through a plug of silica gel $(3 \mathrm{~cm})$ 


\section{Supporting Information}

with AcOEt/hexane $(1: 1,80 \mathrm{~mL})$ as an eluent. The solvent was removed in vacuo, and the residue was purified by column chromatography (99:1 hexane/AcOEt) to provide $172 \mathrm{mg}(99 \%)$ of endo-12a. The enantiomeric excess was determined by HPLC analysis (DAICEL Chiralpak AD-H, 1 : $99 i$ $\mathrm{PrOH} /$ hexane, flow $0.5 \mathrm{~mL} / \mathrm{min}, 35^{\circ} \mathrm{C}$ ) $\mathrm{t}_{\mathrm{R}}=13.3 \mathrm{~min}$ (minor), $14.2 \mathrm{~min}$ (major).

\section{1-Butanoyl-7-endo-butoxy-5-methoxy-8-oxabenzo[c]bicyclo[3.2.1]octan-2-one (endo-12a):}

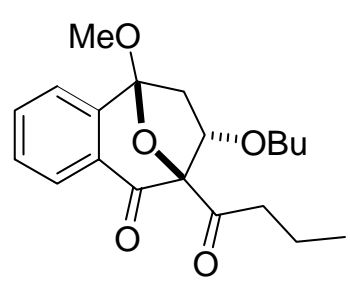

Colorless viscous oil; $[\alpha]_{\mathrm{D}}^{25}+175.8\left(c 1.00, \mathrm{CHCl}_{3}\right)(92 \%$ ee); IR (neat) 3025 , 2963, 2936, 2874, 1730, 1701, 1603, 1458, 1404, 1368, 1302, 1269, 1217, 1171, 1101, 1065, 1040, $1007 \mathrm{~cm}^{-1} ;{ }^{1} \mathrm{H}$ NMR $\left(\mathrm{CDCl}_{3}\right) \delta 0.76(3 \mathrm{H}, \mathrm{t}, J=7.3 \mathrm{~Hz}), 0.95$ $(3 \mathrm{H}, \mathrm{t}, J=7.3 \mathrm{~Hz}), 1.09(2 \mathrm{H}, \mathrm{sext}, J=7.3 \mathrm{~Hz}), 1.20-1.34(2 \mathrm{H}, \mathrm{m}), 1.61-1.76$ $(2 \mathrm{H}, \mathrm{m}), 2.08(1 \mathrm{H}, \mathrm{dd}, J=13.2,1.7 \mathrm{~Hz}), 2.55(1 \mathrm{H}, \mathrm{ddd}, J=6.6,8.1,18.3 \mathrm{~Hz}), 2.60(1 \mathrm{H}, \mathrm{dd}, J=13.2$, $9.5 \mathrm{~Hz}), 2.73(1 \mathrm{H}, \mathrm{ddd}, J=6.6,8.1,18.3 \mathrm{~Hz}), 3.36(1 \mathrm{H}, \mathrm{dt}, J=6.4,9.5 \mathrm{~Hz}), 3.50(1 \mathrm{H}, \mathrm{dt}, J=6.4,9.5$ $\mathrm{Hz}), 3.50(3 \mathrm{H}, \mathrm{s}, \mathrm{OMe}), 4.55(1 \mathrm{H}, \mathrm{dd}, \mathrm{J}=1.7,9.5 \mathrm{~Hz}), 7.45-7.51(2 \mathrm{H}, \mathrm{m}), 7.61-7.66(1 \mathrm{H}, \mathrm{m}), 7.98-$ $8.02(1 \mathrm{H}, \mathrm{m}) ;{ }^{13} \mathrm{C}$ NMR $\left(\mathrm{CDCl}_{3}\right) \delta 13.7\left(\mathrm{CH}_{3}\right), 13.8\left(\mathrm{CH}_{3}\right), 16.5\left(\mathrm{CH}_{2}\right), 19.1\left(\mathrm{CH}_{2}\right), 31.5\left(\mathrm{CH}_{2}\right), 41.2$ $\left(\mathrm{CH}_{2}\right), 43.0\left(\mathrm{CH}_{2}\right), 52.0\left(\mathrm{CH}_{3}\right), 70.5\left(\mathrm{CH}_{2}\right), 77.8(\mathrm{CH}), 95.3(\mathrm{C}), 106.7(\mathrm{C}), 123.1(\mathrm{CH}), 126.5(\mathrm{CH})$, $128.6(\mathrm{CH}), 131.2(\mathrm{C}), 133.7(\mathrm{CH}), 144.1(\mathrm{C}), 189.3(\mathrm{C}), 203.5(\mathrm{C})$; MS (EI) m/z $346\left(\mathrm{M}^{+}\right)$, 303, 246, 231, 186, 175, 161, 147, 129, 117, 103, 91, 71, 61, 49, 39, 26, 13; HRMS (EI) Calcd for $\mathrm{C}_{20} \mathrm{H}_{26} \mathrm{O}_{5}\left(\mathrm{M}^{+}\right)$: 346.1779. Found: 346.1797. Anal. Calcd for $\mathrm{C}_{20} \mathrm{H}_{26} \mathrm{O}_{5}: \mathrm{C}, 69.34 ; \mathrm{H}, 7.56 \%$. Found: $\mathrm{C}, 69.32 ; \mathrm{H}$, 7.64\%. The enantiomeric excess was determined by HPLC analysis (DAICEL Chiralpak AD-H, $1: 99$ $i$-PrOH/hexane, flow $0.5 \mathrm{~mL} / \mathrm{min}, 35^{\circ} \mathrm{C}$ ) $\mathrm{t}_{\mathrm{R}}=13.3 \mathrm{~min}$ (minor), $14.2 \mathrm{~min}$ (major).

\section{1-Butanoyl-7-endo-cyclohexyloxy-5-methoxy-8-oxabenzo[c]bicyclo[3.2.1]octan-2-one}

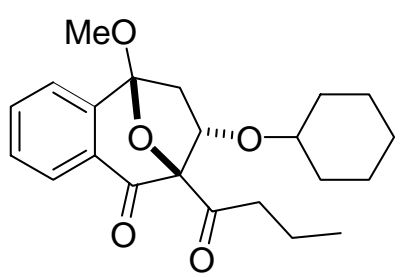
(endo-12d): Colorless viscous oil; $[\alpha]_{\mathrm{D}}^{25}+171.4\left(c\right.$ 1.00, $\left.\mathrm{CHCl}_{3}\right)(93 \%$ ee (endo)); IR (neat) 3021, 2936, 2859, 1728, 1701, 1603, 1522, 1456, 1362, 1300, 1269, 1215, 1169, 1098, $1063 \mathrm{~cm}^{-1} ;{ }^{1} \mathrm{H} \mathrm{NMR}\left(\mathrm{CDCl}_{3}\right) \delta 0.95(3 \mathrm{H}, \mathrm{t}, J$ $=7.3 \mathrm{~Hz}), 0.99-1.86(10 \mathrm{H}, \mathrm{m}), 1.62-1.74(2 \mathrm{H}, \mathrm{m}), 2.04(1 \mathrm{H}, \mathrm{dd}, J=12.9,1.7$

Hz), $2.59(1 \mathrm{H}, \mathrm{dd}, J=12.9,9.5 \mathrm{~Hz}), 2.56(1 \mathrm{H}, \mathrm{ddd}, J=6.4,8.1,18.3 \mathrm{~Hz}), 2.74(1 \mathrm{H}, \mathrm{ddd}, J=6.6,7.8$, $18.3 \mathrm{~Hz}), 3.50(3 \mathrm{H}, \mathrm{s}, \mathrm{OMe}), 3.44-3.54(1 \mathrm{H}, \mathrm{m}), 4.74(1 \mathrm{H}, \mathrm{dd}, J=1.7,9.5 \mathrm{~Hz}), 7.43-7.51(2 \mathrm{H}, \mathrm{m})$, 


\section{Supporting Information}

7.59-7.66 (1H, m), 7.96-8.03 (1H, m); ${ }^{13} \mathrm{C}$ NMR $\left(\mathrm{CDCl}_{3}\right) \delta 13.8\left(\mathrm{CH}_{3}\right), 16.5\left(\mathrm{CH}_{2}\right), 23.7\left(\mathrm{CH}_{2}\right), 23.9$ $\left(\mathrm{CH}_{2}\right), 25.8\left(\mathrm{CH}_{2}\right), 31.5\left(\mathrm{CH}_{2}\right), 32.3(\mathrm{CH}), 41.2\left(\mathrm{CH}_{2}\right), 43.6\left(\mathrm{CH}_{2}\right), 52.0\left(\mathrm{CH}_{3}\right), 74.8\left(\mathrm{CH}_{2}\right), 76.5(\mathrm{CH})$, $95.5(\mathrm{C}), 106.9(\mathrm{C}), 123.2(\mathrm{CH}), 126.5(\mathrm{CH}), 128.6(\mathrm{CH}), 131.5(\mathrm{C}), 133.6(\mathrm{CH}), 144.1(\mathrm{C}), 189.7(\mathrm{C})$, 203.8 (C); MS (EI) m/z $372\left(\mathrm{M}^{+}\right)$, 340, 313, 301, 290, 272, 258, 246, 230, 218, 201, 191, 187, 175, 163, 147, 129, 115, 103, 83, 71, 55, 39, 24, 13; HRMS (EI) Calcd for $\mathrm{C}_{22} \mathrm{H}_{28} \mathrm{O}_{5}\left(\mathrm{M}^{+}\right)$: 372.1935. Found: 372.1953. The enantiomeric excess was determined by HPLC analysis (DAICEL Chiralpak AD-H, 1 : $99 i-\mathrm{PrOH} /$ hexane, flow $0.5 \mathrm{~mL} / \mathrm{min}, 35^{\circ} \mathrm{C}$ ) $\mathrm{t}_{\mathrm{R}}=13.8 \mathrm{~min}$ (minor), $17.8 \mathrm{~min}$ (major).

\section{1-Isobutanoyl-7-endo-cyclohexyloxy-5-methoxy-8-oxabenzo[c]bicyclo[3.2.1]octan-2-one}

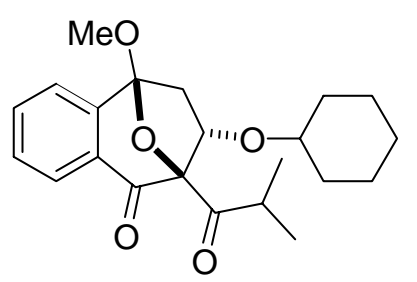

(endo-13d): Colorless viscous oil; $[\alpha]_{\mathrm{D}}^{25}+178.7$ ( c $\left.1.00, \mathrm{CHCl}_{3}\right)(97 \%$ ee (endo)); IR (neat) 3021, 2976, 2936, 2859, 1728, 1699, 1603, 1454, 1300,

$1267,1215,1181,1150,1098,1065,1028,1007 \mathrm{~cm}^{-1} ;{ }^{1} \mathrm{H}$ NMR $\left(\mathrm{CDCl}_{3}\right) \delta$ 0.95-1.84 (10H, m), $1.13(3 \mathrm{H}, \mathrm{d}, J=6.8 \mathrm{~Hz}), 1.15(3 \mathrm{H}, \mathrm{d}, J=6.8 \mathrm{~Hz}), 2.03$ $(1 \mathrm{H}, \mathrm{dd}, J=12.9,1.7 \mathrm{~Hz}), 2.59(1 \mathrm{H}, \mathrm{dd}, J=12.9,9.5 \mathrm{~Hz}), 3.07(1 \mathrm{H}, \mathrm{sept}, J=$ $6.8 \mathrm{~Hz}), 3.50(3 \mathrm{H}, \mathrm{s}), 3.50-3.60(1 \mathrm{H}, \mathrm{m}), 4.67(1 \mathrm{H}, \mathrm{dd}, J=1.7,9.5 \mathrm{~Hz}), 7.43-7.51(2 \mathrm{H}, \mathrm{m}), 7.59-7.67$ $(1 \mathrm{H}, \mathrm{m}), 7.96-8.05(1 \mathrm{H}, \mathrm{m}) ;{ }^{13} \mathrm{C} \mathrm{NMR}\left(\mathrm{CDCl}_{3}\right) \delta 18.4\left(\mathrm{CH}_{3}\right), 18.8\left(\mathrm{CH}_{3}\right), 23.7\left(\mathrm{CH}_{2}\right), 23.9\left(\mathrm{CH}_{2}\right), 25.9$ $\left(\mathrm{CH}_{2}\right), 31.4\left(\mathrm{CH}_{2}\right), 32.3\left(\mathrm{CH}_{2}\right), 37.8(\mathrm{CH}), 43.5\left(\mathrm{CH}_{2}\right), 52.0\left(\mathrm{CH}_{3}\right), 75.6(\mathrm{CH}), 76.4(\mathrm{CH}), 96.1(\mathrm{C})$, $106.7(\mathrm{C}), 123.2(\mathrm{CH}), 126.7(\mathrm{CH}), 128.6(\mathrm{CH}), 131.4(\mathrm{C}), 133.6(\mathrm{CH}), 144.0(\mathrm{C}), 189.2(\mathrm{C}), 208.1(\mathrm{C})$; MS (EI) m/z $372\left(\mathrm{M}^{+}\right), 340,313,301,290,272,258,246,230,215,201,191,187,173,163,147,129$, 115, 103, 83, 71, 55, 39, 24, 13; HRMS (EI) Calcd for $\mathrm{C}_{22} \mathrm{H}_{28} \mathrm{O}_{5}\left(\mathrm{M}^{+}\right)$: 372.1935. Found: 372.1913. Anal. Calcd for $\mathrm{C}_{22} \mathrm{H}_{28} \mathrm{O}_{5}: \mathrm{C}, 70.94 ; \mathrm{H}, 7.58 \%$. Found: $\mathrm{C}, 70.54 ; \mathrm{H}, 7.97 \%$. The enantiomeric excess was determined by HPLC analysis (DAICEL Chiralpak AD-H, $1: 99 i$-PrOH/hexane, flow $0.5 \mathrm{~mL} / \mathrm{min}$, $\left.35^{\circ} \mathrm{C}\right) \mathrm{t}_{\mathrm{R}}=10.3 \min ($ minor), $12.4 \min$ (major).

\section{7-endo-Cyclohexyloxy-5-methoxy-1-pentanoyl-8-oxabenzo[c]bicyclo[3.2.1]octan-2-one}

(endo-14d): Colorless viscous oil; $[\alpha]_{\mathrm{D}}{ }^{25}+180.5$ ( $c$ 1.00, $\left.\mathrm{CHCl}_{3}\right)(93 \%$ ee (endo)); IR (neat) 3020, 2935, $1728,1701,1423,1302,1269,1215,1168,1097,1047,929,771,669 \mathrm{~cm}^{-1} ;{ }^{1} \mathrm{H}$ NMR $\left(\mathrm{CDCl}_{3}\right) \delta 0.91$ $(3 \mathrm{H}, \mathrm{t}, J=7.3 \mathrm{~Hz}), 0.95-1.74(10 \mathrm{H}, \mathrm{m}), 1.34(2 \mathrm{H}, \mathrm{m}), 1.64(2 \mathrm{H}, \mathrm{m}) 2.03(1 \mathrm{H}, \mathrm{dd}, J=1.7,12.9 \mathrm{~Hz}), 2.56$ 


\section{Supporting Information}

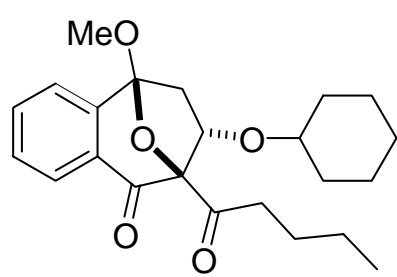

$(1 \mathrm{H}, \mathrm{m}), 2.58(1 \mathrm{H}, \mathrm{dd}, J=9.5,12.9 \mathrm{~Hz}), 2.75(1 \mathrm{H}, \mathrm{ddd}, J=6.3,8.3,18.0$ $\mathrm{Hz}), 3.40-3.54(1 \mathrm{H}, \mathrm{m}), 3.50(3 \mathrm{H}, \mathrm{s}), 4.73(1 \mathrm{H}, \mathrm{dd}, J=1.7,9.5 \mathrm{~Hz}), 7.42-$ $7.50(2 \mathrm{H}, \mathrm{m}), 7.59-7.66(1 \mathrm{H}, \mathrm{m}), 7.95-8.02(1 \mathrm{H}, \mathrm{m}) ;{ }^{13} \mathrm{C} \mathrm{NMR}\left(\mathrm{CDCl}_{3}\right) \delta$ $13.9\left(\mathrm{CH}_{3}\right), 22.2\left(\mathrm{CH}_{2}\right), 23.6\left(\mathrm{CH}_{2}\right), 23.8\left(\mathrm{CH}_{2}\right), 25.1\left(\mathrm{CH}_{2}\right), 25.8\left(\mathrm{CH}_{2}\right)$, 31.4 $\left(\mathrm{CH}_{2}\right), 32.2\left(\mathrm{CH}_{2}\right), 38.9\left(\mathrm{CH}_{2}\right), 43.5\left(\mathrm{CH}_{2}\right), 51.9\left(\mathrm{CH}_{3}\right), 74.8(\mathrm{CH}), 76.4(\mathrm{CH}), 95.5(\mathrm{C}), 106.8(\mathrm{C})$, $123.1(\mathrm{CH}), 126.4(\mathrm{CH}), 128.5(\mathrm{CH}), 131.4(\mathrm{C}), 133.6(\mathrm{CH}), 144.1(\mathrm{C}), 189.7(\mathrm{C}), 203.9(\mathrm{C})$; MS (EI) m/z $386\left(M^{+}\right), 354,327,304,286,272,260,244,231,218,201,200,187,173,161,147,129,115,103$, 83, 71, 57, 37, 26, 13; HRMS (EI) Calcd for $\mathrm{C}_{23} \mathrm{H}_{30} \mathrm{O}_{5}\left(\mathrm{M}^{+}\right)$: 386.2092. Found: 386.2112. The enantiomeric excess was determined by HPLC analysis (DAICEL Chiralpak AD-H, 1 : $99 i$ $\mathrm{PrOH} /$ hexane, flow $0.5 \mathrm{~mL} / \mathrm{min}, 35^{\circ} \mathrm{C}$ ) $\mathrm{t}_{\mathrm{R}}=13.3 \mathrm{~min}$ (minor), $17.8 \mathrm{~min}$ (major).

7-endo-Cyclohexyloxy-5-methoxy-1-(3-methylbutanoyl)-8-oxabenzo[c]bicyclo[3.2.1]-

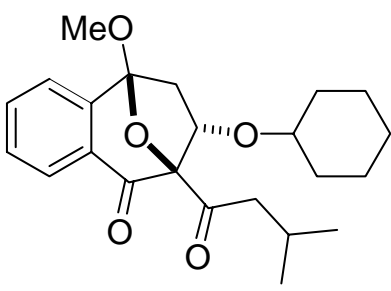

octan-2-one (endo-15d): Colorless viscous oil; $[\alpha]_{\mathrm{D}}{ }^{25}+177.3$ (c 1.00, $\left.\mathrm{CHCl}_{3}\right)(88 \%$ ee (endo)); IR (neat) 3568, 3021, 2936, 2858, 2401, 1728, $1703,1602,1415,1366,1362,1269,1215,1169,1169,1098,1064,758$, $530 \mathrm{~cm}^{-1} ;{ }^{1} \mathrm{H} \mathrm{NMR}\left(\mathrm{CDCl}_{3}\right) \delta 0.84-1.70(10 \mathrm{H}, \mathrm{m}), 0.94(3 \mathrm{H}, \mathrm{d}, J=6.5 \mathrm{~Hz})$, $0.97(3 \mathrm{H}, \mathrm{d}, J=6.8 \mathrm{~Hz}), 2.06(1 \mathrm{H}, \mathrm{dd}, J=13.2,1.7 \mathrm{~Hz}), 2.25(1 \mathrm{H}, \mathrm{m}), 2.47$ $(1 \mathrm{H}, \mathrm{dd}, J=6.5,17.8 \mathrm{~Hz}) 2.58(1 \mathrm{H}, \mathrm{dd}, J=13.2,9.2 \mathrm{~Hz}), 2.63(1 \mathrm{H}, \mathrm{dd}, J=6.8,17.8 \mathrm{~Hz}), 3.42-3.54$ (1H, m), $3.49(3 \mathrm{H}, \mathrm{s}), 4.72(1 \mathrm{H}, \mathrm{dd}, J=1.7,9.2 \mathrm{~Hz}), 7.41-7.52(2 \mathrm{H}, \mathrm{m}), 7.58-7.67(1 \mathrm{H}, \mathrm{m}), 7.94-8.03$ $(1 \mathrm{H}, \mathrm{m}) ;{ }^{13} \mathrm{C} \mathrm{NMR}\left(\mathrm{CDCl}_{3}\right) \delta 22.6\left(\mathrm{CH}_{3}\right), 22.7\left(\mathrm{CH}_{3}\right), 23.7(\mathrm{CH}), 23.7\left(\mathrm{CH}_{2}\right), 23.8\left(\mathrm{CH}_{2}\right), 25.8\left(\mathrm{CH}_{2}\right)$, $31.5\left(\mathrm{CH}_{2}\right), 32.2\left(\mathrm{CH}_{2}\right), 43.5\left(\mathrm{CH}_{2}\right), 48.1\left(\mathrm{CH}_{2}\right), 52.0\left(\mathrm{CH}_{3}\right), 74.8(\mathrm{CH}), 76.4(\mathrm{CH}), 95.4(\mathrm{C}), 106.8$ (C),123.1 (CH), $126.5(\mathrm{CH}), 128.6(\mathrm{CH}), 131.5(\mathrm{C}), 133.6(\mathrm{CH}), 144.1(\mathrm{C}), 189.6(\mathrm{C}), 203.3(\mathrm{C})$; MS (EI) $\mathrm{m} / \mathrm{z} 386\left(\mathrm{M}^{+}\right), 354,327,304,286,272,261,244,232,219,201,200,187,173,161,147,129,115$, 103, 85, 69, 57, 39, 26, 13; HRMS (EI) Calcd for $\mathrm{C}_{23} \mathrm{H}_{30} \mathrm{O}_{5}\left(\mathrm{M}^{+}\right)$: 386.2092. Found: 386.2106. The enantiomeric excess was determined by HPLC analysis (DAICEL Chiralpak AD-H, $0.5: 99.5 i$ $\mathrm{PrOH} /$ hexane, flow $0.5 \mathrm{~mL} / \mathrm{min}, 35^{\circ} \mathrm{C}$ ) $\mathrm{t}_{\mathrm{R}}=14.9 \mathrm{~min}$ (minor), $18.0 \mathrm{~min}$ (major). 


\section{Supporting Information}

7-endo-Cyclohexyloxy-1-hexanoyl-5-methoxy-8-oxabenzo[c]bicyclo[3.2.1]-octan-2-one

(endo-16d): Colorless viscous oil; $[\alpha]_{\mathrm{D}}^{25}+154.1$ (c $0.80, \mathrm{CHCl}_{3}$ ) (84\% ee, (endo)); IR (neat) 3021,

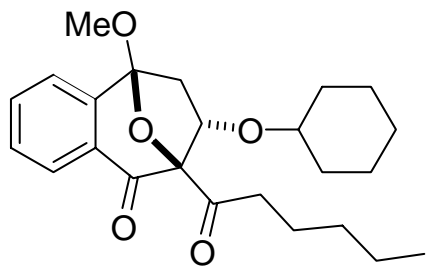
$2934,2859,1730,1701,1651,1603,1507,1456,1362,1300,1269,1215$, 1167, 1098, $1065 \mathrm{~cm}^{-1} ;{ }^{1} \mathrm{H} \mathrm{NMR}\left(\mathrm{CDCl}_{3}\right) \delta 0.89(3 \mathrm{H}, \mathrm{t}, J=7.3 \mathrm{~Hz}), 1.01-$ $1.77(10 \mathrm{H}, \mathrm{m}), 1.04,(2 \mathrm{H}, \mathrm{m}), 1.31(2 \mathrm{H}, \mathrm{m}), 1.65(2 \mathrm{H}, \mathrm{m}), 2.03(1 \mathrm{H}, \mathrm{dd}, J=$ $1.7,12.9 \mathrm{~Hz}), 2.56(1 \mathrm{H}$, ddd, $J=6.3,8.1,18.3 \mathrm{~Hz}), 2.58(1 \mathrm{H}, \mathrm{dd}, J=9.3$, $12.9 \mathrm{~Hz}), 2.75(1 \mathrm{H}, \mathrm{ddd}, J=6.6,8.3,18.3 \mathrm{~Hz}), 3.49(3 \mathrm{H}, \mathrm{s}), 3.45-3.52(1 \mathrm{H}, \mathrm{m}), 4.73(1 \mathrm{H}, \mathrm{dd}, J=1.7$, $9.3 \mathrm{~Hz}), 7.44-7.48(2 \mathrm{H}, \mathrm{m}), 7.60-7.64(1 \mathrm{H}, \mathrm{m}), 7.96-8.00(1 \mathrm{H}, \mathrm{m}) ;{ }^{13} \mathrm{C} \mathrm{NMR}\left(\mathrm{CDCl}_{3}\right) \delta 14.1\left(\mathrm{CH}_{3}\right)$, $22.6\left(\mathrm{CH}_{2}\right), 22.8\left(\mathrm{CH}_{2}\right), 23.8\left(\mathrm{CH}_{2}\right), 23.9\left(\mathrm{CH}_{2}\right), 25.9\left(\mathrm{CH}_{2}\right), 31.4\left(\mathrm{CH}_{2}\right), 31.5\left(\mathrm{CH}_{2}\right), 32.3\left(\mathrm{CH}_{2}\right), 39.4$ $\left(\mathrm{CH}_{2}\right), 43.6\left(\mathrm{CH}_{2}\right), 52.1\left(\mathrm{CH}_{3}\right), 74.9(\mathrm{CH}), 76.5(\mathrm{CH}), 95.6(\mathrm{C}), 106.9(\mathrm{C}), 123.2(\mathrm{CH}), 126.6(\mathrm{CH})$, $128.6(\mathrm{CH}), 131.6(\mathrm{C}), 133.7(\mathrm{CH}), 144.1(\mathrm{C}), 189.8(\mathrm{C}), 204.1(\mathrm{C})$; MS (EI) m/z $400\left(\mathrm{M}^{+}\right), 368,341$, $318,301,275,258,246,231,215,201,187,173,159,145,129,116,103,83,69,55,37,24$; HRMS (EI) Calcd for Calcd for $\mathrm{C}_{24} \mathrm{H}_{32} \mathrm{O}_{5}\left(\mathrm{M}^{+}\right)$: 400.2248. Found: 400.2242. The enantiomeric excess was determined by HPLC analysis (DAICEL Chiralpak AD-H, $1: 99 i$-PrOH/hexane, flow $0.5 \mathrm{~mL} / \mathrm{min}$, $\left.35^{\circ} \mathrm{C}\right) \mathrm{t}_{\mathrm{R}}=42.0 \mathrm{~min}($ minor), $55.7 \mathrm{~min}$ (major).

\section{1-(Cylohexylcarbonyl)-7-endo-cyclohexyloxy-5-methoxy-8-oxabenzo[c]bicyclo[3.2.1]-}

octan-2-one (endo-17d): Colorless prisms (hexane); mp 95-96 ${ }^{\circ} \mathrm{C} ;[\alpha]_{\mathrm{D}}{ }^{25}+163.7\left(c 1.00, \mathrm{CHCl}_{3}\right)$

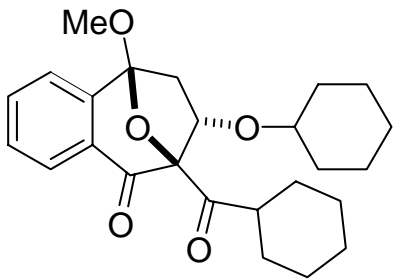
(96\% ee (endo)); IR (KBr) 3021, 2936, 2857, 1728, 1699, 1557, 1539, 1520, 1454, 1300, 1267, 1217, 1167, 1098, 1051, $974 \mathrm{~cm}^{-1} ;{ }^{1} \mathrm{H}$ NMR $\left(\mathrm{CDCl}_{3}\right) \delta$ 0.98-1.92 (20H, m), $2.01(1 \mathrm{H}, \mathrm{dd}, J=1.7,12.9 \mathrm{~Hz}), 2.58(1 \mathrm{H}, \mathrm{dd}, J=9.3$,

$12.9 \mathrm{~Hz}), 2.82(1 \mathrm{H}, \mathrm{tt}, J=3.2,11.2 \mathrm{~Hz}), 3.50(3 \mathrm{H}, \mathrm{s}), 3.52-3.56(1 \mathrm{H}, \mathrm{m})$, $4.66(1 \mathrm{H}, \mathrm{dd}, J=1.7,9.3 \mathrm{~Hz}), 7.44-7.48(2 \mathrm{H}, \mathrm{m}), 7.60-7.64(1 \mathrm{H}, \mathrm{m}), 7.98-8.00(1 \mathrm{H}, \mathrm{m})$; ${ }^{13} \mathrm{C} \mathrm{NMR}$ $\left(\mathrm{CDCl}_{3}\right) \delta 23.8\left(\mathrm{CH}_{2}\right), 23.9\left(\mathrm{CH}_{2}\right), 25.7\left(\mathrm{CH}_{2}\right), 25.9\left(\mathrm{CH}_{2}\right), 26.0\left(\mathrm{CH}_{2}\right), 28.5\left(\mathrm{CH}_{2}\right), 28.9\left(\mathrm{CH}_{2}\right), 31.5$ $\left(\mathrm{CH}_{2}\right), 32.4\left(\mathrm{CH}_{2}\right), 43.5\left(\mathrm{CH}_{2}\right), 47.7(\mathrm{CH}), 52.0\left(\mathrm{CH}_{3}\right), 75.5(\mathrm{CH}), 76.4(\mathrm{CH}), 96.0(\mathrm{C}), 106.7(\mathrm{C}), 123.2$ (CH), $126.7(\mathrm{CH}), 128.6(\mathrm{CH}), 131.5(\mathrm{C}), 133.6(\mathrm{CH}), 144.0(\mathrm{C}), 189.3(\mathrm{C}), 207.1(\mathrm{C}) ; \mathrm{MS}(\mathrm{EI}) \mathrm{m} / \mathrm{z}$ $412\left(\mathrm{M}^{+}\right), 380,353,330,287,270,201,187,174,161,147,129,103,83,55,37,24$; HRMS (EI) Calcd for $\mathrm{C}_{25} \mathrm{H}_{32} \mathrm{O}_{5}\left(\mathrm{M}^{+}\right)$: 412.2248. Found: 412.2234 The enantiomeric excess was determined by HPLC 


\section{Supporting Information}

analysis (DAICEL Chiralpak AD-H, $1: 99 i$-PrOH/hexane, flow $0.5 \mathrm{~mL} / \mathrm{min}, 35^{\circ} \mathrm{C}$ ) $\mathrm{t}_{\mathrm{R}}=16.0 \mathrm{~min}$ (major), $19.5 \min$ (minor).

7-endo-Cyclohexyloxy-5-methoxy-1-(phenylacetyl)-8-oxabenzo[c]bicyclo[3.2.1]-octan-2one (endo-18d): Colorless viscous oil; $[\alpha]_{\mathrm{D}}{ }^{25}+165.7$ (c 1.00, $\left.\mathrm{CHCl}_{3}\right)(92 \%$ ee (endo)); IR (neat) 3020,

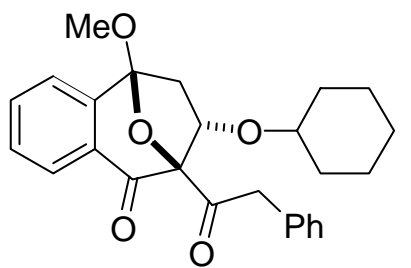
$2938,1734,1701,1302,1269,1215,1165,1098,1067,1028,976,669 \mathrm{~cm}^{-1}$; ${ }^{1} \mathrm{H}$ NMR $\left(\mathrm{CDCl}_{3}\right) \delta 0.84-1.72(10 \mathrm{H}, \mathrm{m}), 2.05(1 \mathrm{H}, \mathrm{dd}, J=13.1,1.7 \mathrm{~Hz}), 2.60$ $(1 \mathrm{H}, \mathrm{dd}, J=13.1,9.5 \mathrm{~Hz}), 3.41-3.56(1 \mathrm{H}, \mathrm{m}), 3.88(1 \mathrm{H}, \mathrm{d}, J=17.1 \mathrm{~Hz}), 4.06$ $(1 \mathrm{H}, \mathrm{d}, J=17.1 \mathrm{~Hz}), 4.75(1 \mathrm{H}, \mathrm{dd}, J=1.7,9.5 \mathrm{~Hz}), 7.44-7.53(2 \mathrm{H}, \mathrm{m}), 7.61-$ $7.67(1 \mathrm{H}, \mathrm{m}), 7.96-8.04(1 \mathrm{H}, \mathrm{m}) ;{ }^{13} \mathrm{C} \mathrm{NMR}\left(\mathrm{CDCl}_{3}\right) \delta 23.6\left(\mathrm{CH}_{2}\right), 23.8\left(\mathrm{CH}_{2}\right), 25.8\left(\mathrm{CH}_{2}\right), 31.3\left(\mathrm{CH}_{2}\right)$, $32.2\left(\mathrm{CH}_{2}\right), 43.6\left(\mathrm{CH}_{2}\right), 45.9\left(\mathrm{CH}_{2}\right), 52.1\left(\mathrm{CH}_{3}\right), 74.8(\mathrm{CH}), 76.4(\mathrm{CH}), 95.7(\mathrm{C}), 107.0(\mathrm{C}), 123.2(\mathrm{CH})$, $126.5(\mathrm{CH}), 126.7(\mathrm{CH}), 128.2(\mathrm{CH}), 128.6(\mathrm{CH}), 129.8(\mathrm{CH}), 131.4(\mathrm{C}), 133.2(\mathrm{C}), 133.7(\mathrm{CH}), 144.0$ (C), 189.5 (C), 201.1 (C); MS (EI) m/z $420\left(\mathrm{M}^{+}\right)$, 388, 360, 338, 307, 294, 278, 262, 247, 234, 219, 203, 187, 173, 160, 147, 129, 104, 91, 77, 55, 37, 24; HRMS (EI) Calcd for $\mathrm{C}_{26} \mathrm{H}_{28} \mathrm{O}_{5}\left(\mathrm{M}^{+}\right): 420.1935$. Found: 420.1942. The enantiomeric excess was determined by HPLC analysis (DAICEL Chiralpak AD-H, $1: 99 i$-PrOH/hexane, flow $0.5 \mathrm{~mL} / \mathrm{min}, 35^{\circ} \mathrm{C}$ ) $t_{\mathrm{R}}=24.2 \mathrm{~min}$ (minor), $39.0 \mathrm{~min}$ (major).

\section{7-endo-Cyclohexyloxy-5-methoxy-1-(3-phenylpropanoyl)-8-oxabenzo[c]bicyclo[3.2.1]-}

octan-2-one (endo-19d): Colorless viscous oil; $[\alpha]_{\mathrm{D}}^{25}+135.1$ (c 1.00, $\left.\mathrm{CHCl}_{3}\right)(77 \%$ ee (endo)); IR

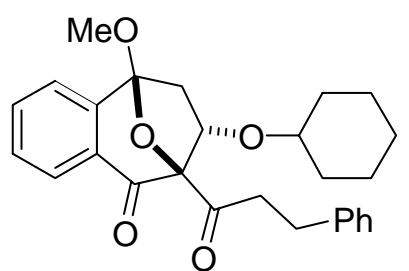
(neat) $2935,2859,1730,1705,1602,1497,1454,1362,1302,1269,1215$, $1165,1096,1046,754,700,505 \mathrm{~cm}^{-1} ;{ }^{1} \mathrm{H}$ NMR $\left(\mathrm{CDCl}_{3}\right) \delta 0.83-1.84(10 \mathrm{H}$ m), $2.03(1 \mathrm{H}, \mathrm{dd}, J=13.1,1.7 \mathrm{~Hz}), 2.56(1 \mathrm{H}, \mathrm{dd}, J=13.1,9.5 \mathrm{~Hz}), 2.89(1 \mathrm{H}$, m), $2.99(2 \mathrm{H}, \mathrm{m}), 3.07(1 \mathrm{H}, \mathrm{m}), 3.50(3 \mathrm{H}, \mathrm{s}), 3.40-3.54(1 \mathrm{H}, \mathrm{m}), 4.71(1 \mathrm{H}, \mathrm{dd}$, $J=1.7,9.5 \mathrm{~Hz}), 7.39-7.53(2 \mathrm{H}, \mathrm{m}), 7.57-7.66(1 \mathrm{H}, \mathrm{m}), 7.93-8.01(1 \mathrm{H}, \mathrm{m}) ;{ }^{13} \mathrm{C} \mathrm{NMR}\left(\mathrm{CDCl}_{3}\right) \delta 23.7$ $\left(\mathrm{CH}_{2}\right), 23.8\left(\mathrm{CH}_{2}\right), 25.8\left(\mathrm{CH}_{2}\right), 29.2\left(\mathrm{CH}_{2}\right), 31.5\left(\mathrm{CH}_{2}\right), 32.3\left(\mathrm{CH}_{2}\right), 41.0\left(\mathrm{CH}_{2}\right), 43.7\left(\mathrm{CH}_{2}\right), 52.1\left(\mathrm{CH}_{3}\right)$, $74.9(\mathrm{CH}), 76.6(\mathrm{CH}), 95.4(\mathrm{C}), 107.0(\mathrm{C}), 123.2(\mathrm{CH}), 125.8(\mathrm{CH}), 126.5(\mathrm{CH}), 128.1(\mathrm{CH}), 128.2$ (CH), $128.6(\mathrm{CH}), 131.5(\mathrm{C}), 133.7(\mathrm{CH}), 140.8(\mathrm{C}), 144.0,(\mathrm{C}) 189.7(\mathrm{C}), 202.8(\mathrm{C}) ; \mathrm{MS}(\mathrm{EI}) \mathrm{m} / \mathrm{z} 434$ $\left(\mathrm{M}^{+}\right), 402, .375,352,334,309,292,279,263,248,231,217,201,187,173,149,129,105,91,71,57$, 


\section{Supporting Information}

39, 26, 13; HRMS (EI) Calcd for $\mathrm{C}_{27} \mathrm{H}_{30} \mathrm{O}_{5}\left(\mathrm{M}^{+}\right)$: 434.2092. Found: 434.2104. The enantiomeric excess was determined by HPLC analysis (DAICEL Chiralpak AD-H, 2 : $98 i$-PrOH/hexane, flow 0.2 $\mathrm{mL} / \min , 35^{\circ} \mathrm{C}$ ) $\mathrm{t}_{\mathrm{R}}=44.4 \min ($ minor), $47.1 \mathrm{~min}$ (major).

endo-Cycloadduct 22: Colorless viscous oil; $[\alpha]_{\mathrm{D}}{ }^{25}-147.1\left(c\right.$ 0.26, $\left.\mathrm{CHCl}_{3}\right)(59 \%$ ee (endo));<smiles>COC12OC(C(=O)c3ccccc31)[C@@H]1CCO[C@@H]12</smiles>
IR (neat) 2976, 2951, 2890, 2845, 2361, 2340, 1713, 1601, 1456, 1331, 1310, 1290, $1246,1200,1169,1088,1061,1044,1013 \mathrm{~cm}^{-1} ;{ }^{1} \mathrm{H}$ NMR $\left(\mathrm{CDCl}_{3}\right) \delta 1.42-1.52$ $(1 \mathrm{H}, \mathrm{m}), 1.89-2.02(1 \mathrm{H}, \mathrm{m}), 2.73(1 \mathrm{H}, \mathrm{q}, J=8.1 \mathrm{~Hz}), 3.28(1 \mathrm{H}, \mathrm{dt}, J=8.8,2.4$ $\mathrm{Hz}), 3.45(3 \mathrm{H}, \mathrm{s}), 3.65(1 \mathrm{H}, \mathrm{dt}, J=8.8,4.4 \mathrm{~Hz}), 4.91(1 \mathrm{H}, \mathrm{d}, J=7.3 \mathrm{~Hz}), 5.15(1 \mathrm{H}$, $\mathrm{dd}, J=8.1,7.3 \mathrm{~Hz}), 7.43-7.54(2 \mathrm{H}, \mathrm{m}), 7.61-7.68(1 \mathrm{H}, \mathrm{m}), 8.03-8.08(1 \mathrm{H}, \mathrm{m}) ;{ }^{13} \mathrm{C} \mathrm{NMR}\left(\mathrm{CDCl}_{3}\right) \delta$ $26.6\left(\mathrm{CH}_{2}\right), 52.3\left(\mathrm{CH}_{3}\right), 52.9(\mathrm{CH}), 72.0\left(\mathrm{CH}_{2}\right), 83.1(\mathrm{CH}), 83.7(\mathrm{CH}), 108.4(\mathrm{C}), 125.2(\mathrm{CH})$, 126.6, (CH), $129.0(\mathrm{CH}), 132.3(\mathrm{C}), 133.1(\mathrm{CH}), 141.2(\mathrm{C}), 192.1(\mathrm{C})$; MS (EI) m/z $246\left(\mathrm{M}^{+}\right)$, 217, 202, 187, $173,161,148,139,128,115,102,91,76,63,55,47,35,24,13$. Anal. Calcd for $\mathrm{C}_{14} \mathrm{H}_{14} \mathrm{O}_{4}$ : C, 68.28; $\mathrm{H}$, 5.73\%. Found : C, 68.28; H, 5.68\%. The enantiomeric excess was determined by HPLC analysis (DAICEL Chiralpak AD-H, $1: 9 i$-PrOH/hexane, flow $0.5 \mathrm{~mL} / \mathrm{min}, 35^{\circ} \mathrm{C}$ ) $\mathrm{t}_{\mathrm{R}}=17.9 \mathrm{~min}$ (major), 18.8 $\min (\operatorname{minor})$.

exo-7-Butoxy-5-methyl-8-oxabicyclo[3.2.1]octan-2-one (exo-21): Colorless viscous oil; $[\alpha]_{\mathrm{D}}$

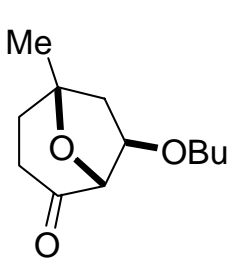

$25+31.6\left(\mathrm{CHCl}_{3}, c\right.$ 1.00)(68\% ee (exo)); IR (neat) 4216, 3447, 3020, 2934, 2402, 2350, 1730, 1682, 1633, 1520, 1454, 1217, 1093, 929, 772, $669 \mathrm{~cm}^{-1} ;{ }^{1} \mathrm{H}$ NMR $\left(\mathrm{CDCl}_{3}\right) \delta 0.91(3 \mathrm{H}, \mathrm{t}, J=7.3 \mathrm{~Hz}), 1.35(2 \mathrm{H}$, sext, $J=7.3 \mathrm{~Hz}), 1.51(2 \mathrm{H}, \mathrm{m}), 1.53$ $(3 \mathrm{H}, \mathrm{s}), 1.81(1 \mathrm{H}, \mathrm{ddd}, J=2.2,8.0,13.4 \mathrm{~Hz}), 1.87(1 \mathrm{H}, \mathrm{dd}, J=2.7,13.9 \mathrm{~Hz}), 2.08$ $(1 \mathrm{H}, \mathrm{m}), 2.25(1 \mathrm{H}, \mathrm{ddd}, J=8.3 .9 .8,17.6 \mathrm{~Hz}), 2.38(1 \mathrm{H}, \mathrm{dd}, J=7.6,13.9 \mathrm{~Hz}), 2.49(1 \mathrm{H}, \mathrm{m}), 3.35(1 \mathrm{H}$, $\mathrm{dt}, J=6.8,9.3 \mathrm{~Hz}), 3.45(1 \mathrm{H}, \mathrm{dt}, J=6.8,9.3 \mathrm{~Hz}), 3.95(1 \mathrm{H}, \mathrm{dd}, J=2.7,7.6 \mathrm{~Hz}), 4.29(1 \mathrm{H}, \mathrm{s}) ;{ }^{13} \mathrm{C} \mathrm{NMR}$ $\left(\mathrm{CDCl}_{3}\right) \delta 14.0\left(\mathrm{CH}_{3}\right), 19.2\left(\mathrm{CH}_{2}\right), 26.1\left(\mathrm{CH}_{3}\right), 31.7\left(\mathrm{CH}_{2}\right), 34.0\left(\mathrm{CH}_{2}\right), 36.3\left(\mathrm{CH}_{2}\right), 44.0\left(\mathrm{CH}_{2}\right), 69.6$ $\left(\mathrm{CH}_{2}\right), 80.8(\mathrm{C}), 82.8(\mathrm{CH}), 87.4(\mathrm{CH}), 206.2(\mathrm{C})$; MS (EI) m/z $212\left(\mathrm{M}^{+}\right), 184,156,140,127,121,112$, $109,99,93,82,75,69,62,56,49,41,35,24,13$. Anal. Calcd for $\mathrm{C}_{12} \mathrm{H}_{20} \mathrm{O}_{3}: \mathrm{C}, 67.89 ; \mathrm{H} \mathrm{9.50 \% ..}$ Found: C, 67.90, .H 9.47\%. The enantiomeric excess was determined by ${ }^{1} \mathrm{H}$ NMR analysis after 


\section{Supporting Information}

conversion to the corresponding acetal in the reaction with $(R, R)$-hydrobenzoin in the presence of PPTS. Relative stereochmistry (exo/endo) of the products could be determined by ${ }^{1} \mathrm{H}$ NMR analysis on the basis of a coupling constant between $\mathrm{H}-1$ and $\mathrm{H}-7$ which reported previously (exo: $0 \mathrm{~Hz}$, endo: $6.8 \mathrm{~Hz}$ ). ${ }^{7}$

endo-7-Butoxy-5-methyl-8-oxabicyclo[3.2.1]octan-2-one (endo-21): Although only a trace

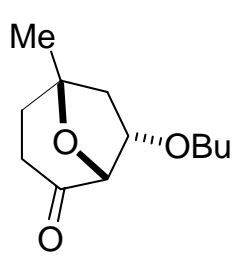

amount of minor endo-21 could separate by chromatography, it could characterize by

${ }^{1} \mathrm{H}$ NMR. ${ }^{1} \mathrm{H}$ NMR $\left(\mathrm{CDCl}_{3}\right) \delta 0.91(3 \mathrm{H}, \mathrm{t}, J=7.6 \mathrm{~Hz}), 1.24-1.33(2 \mathrm{H}, \mathrm{m}), 1.40(3 \mathrm{H}$, s), 1.43-1.50 (2H, m), $1.95-2.01(2 \mathrm{H}, \mathrm{m}), 2.10(1 \mathrm{H}, \mathrm{m}), 2.23(1 \mathrm{H}, \mathrm{dd}, J=9.2,13.4$ $\mathrm{Hz}), 2.44(1 \mathrm{H}, \mathrm{m}), 2.65(1 \mathrm{H}, \mathrm{ddd}, J=8.7,11.7,17.0 \mathrm{~Hz}), 3.35(2 \mathrm{H}, \mathrm{dt}, \mathrm{J}=6.4,1.9 \mathrm{~Hz})$, $4.31(1 \mathrm{H}, \mathrm{d}, J=6.8 \mathrm{~Hz}), 4.34(1 \mathrm{H}, \mathrm{ddd}, J=3.9,6.8,9.8 \mathrm{~Hz})$.

\section{Spectroscopic data of new diazo compounds}

These diazo compounds mostly did not give satisfactory elemental analysis or HRMS data because of instability of the compounds (easily releasing nitrogen).

4: Yellow solid; $\mathrm{mp} 52-53^{\circ} \mathrm{C}$; IR (KBr) 3021, 2961, 2930, 2874, 2402, 2126, 1721, $1657 \mathrm{~cm}^{-1}$;<smiles>CCCC(=O)C(=O)C(=O)c1ccccc1C(C)=O</smiles>
${ }^{1} \mathrm{H} \mathrm{NMR}\left(\mathrm{CDCl}_{3}\right) \delta 0.98(3 \mathrm{H}, \mathrm{t}, J=7.3 \mathrm{~Hz}), 1.70(2 \mathrm{H}$, sext, $J=7.3 \mathrm{~Hz}), 2.83$ $(2 \mathrm{H}, \mathrm{t}, J=7.3 \mathrm{~Hz}), 3.89(3 \mathrm{H}, \mathrm{s}), 7.33-7.40(1 \mathrm{H}, \mathrm{m}), 7.53-7.60(1 \mathrm{H}, \mathrm{m}), 7.61-$ $7.68(1 \mathrm{H}, \mathrm{m}), 8.03-8.08(1 \mathrm{H}, \mathrm{m}) ; \mathrm{MS}(\mathrm{EI}) \mathrm{m} / \mathrm{z} 274\left(\mathrm{M}^{+}\right), 246,231,218,203$, $186,176,163,147,133,115,104,92,77,69,55,47,39,24$. Anal. Calcd for $\mathrm{C}_{14} \mathrm{H}_{14} \mathrm{~N}_{2} \mathrm{O}_{4}: \mathrm{C}, 61.31 ; \mathrm{H}$, 5.14; N, 10.21\%. Found: C, 61.29; H, 5.12, N, 10.26\%.

5: Pale yellow oil; IR (neat) 3023, 2976, 2361, 2340, 2124, 1723, 1657, $1597 \mathrm{~cm}^{-1}$; ${ }^{1} \mathrm{H}$ NMR<smiles>COC(=O)C(=NC(=O)C(C)C)C(=O)c1ccccc1OC</smiles>
$\left(\mathrm{CDCl}_{3}\right) \delta 1.20(6 \mathrm{H}, \mathrm{d}, J=6.8 \mathrm{~Hz}), 3.51(1 \mathrm{H}$, sept, $J=6.8 \mathrm{~Hz}), 3.88(3 \mathrm{H}, \mathrm{s})$, 7.34-7.39 (1H, m), 7.51-7.58 (1H, m), 7.60-7.66 (1H, m), 8.02-8.07 (1H, m); MS (EI) m/z $274\left(\mathrm{M}^{+}\right), 269,246,231,218,203,188,175,163,147,133,119$, 104, 92, 76, 63, 55, 47, 35, 24; HRMS (EI) Calcd for $\mathrm{C}_{14} \mathrm{H}_{14} \mathrm{~N}_{2} \mathrm{O}_{5}\left(\mathrm{M}^{+}\right): 274.0953$. Found: 274.0911 .

6: Pale yellow oil; IR (neat) 3427, 2958, 2874, 2359, 2119, 1726, 1660, 1597, 1575, 1487, 1435, 1284, 1196, 1138, 1084, 1041, 964, 902, 771, $680 \mathrm{~cm}^{-1} ;{ }^{1} \mathrm{H} \mathrm{NMR}\left(\mathrm{CDCl}_{3}\right) \delta 0.98(3 \mathrm{H}, \mathrm{t}, J=7.3$ 


\section{Supporting Information}

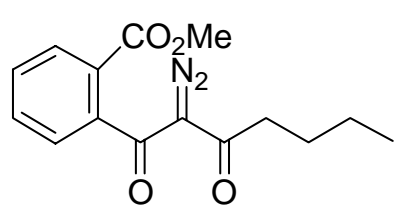

$\mathrm{Hz}), 1.37(2 \mathrm{H}$, sext, $J=7.3 \mathrm{~Hz}), 1.69(2 \mathrm{H}$, quint, $J=7.3 \mathrm{~Hz}), 2.75(2 \mathrm{H}, \mathrm{t}, J$ = 7.3 Hz), $3.89(3 \mathrm{H}, \mathrm{s}), 7.33-7.39(1 \mathrm{H}, \mathrm{m}), 7.50-7.59(1 \mathrm{H}, \mathrm{m}), 7.60-7.67$

$(1 \mathrm{H}, \mathrm{m}), \quad 8.01-8.07(\mathrm{H}, \mathrm{m}) ;{ }^{13} \mathrm{C} \mathrm{NMR}\left(\mathrm{CDCl}_{3}\right) \delta 14.0\left(\mathrm{CH}_{3}\right), 22.4\left(\mathrm{CH}_{2}\right)$, $26.3\left(\mathrm{CH}_{2}\right), 40.9\left(\mathrm{CH}_{2}\right), 52.7\left(\mathrm{CH}_{3}\right), 85.6(\mathrm{C}), 126.2(\mathrm{CH}), 127.6(\mathrm{C}), 130.0$ (CH), $130.5(\mathrm{CH}), 132.8(\mathrm{CH}), 140.4(\mathrm{C}), 165.6(\mathrm{C}), 186.1(\mathrm{C}), 191.8(\mathrm{C}) ; \mathrm{MS}(\mathrm{EI}) \mathrm{m} / \mathrm{z} 260\left(\mathrm{M}^{+}-28\right)$, 245, 229, 218, 203, 190, 176, 164, 147, 133, 115, 104, 92, 77, 66, 55, 37, 24, 13.

7: Pale yellow oil; IR (neat) 3027, 2959, 2874, 2444, 2349, 2122, 1724, 1659, 1597, 1578,<smiles>COC(=O)c1ccccc1C(=O)C(=O)CC(C)C</smiles>
$1487,1437,1369,1311,1284,1197,1168,1138,1084,1043,962,926,682$ $\mathrm{cm}^{-1} ;{ }^{1} \mathrm{H} \mathrm{NMR}\left(\mathrm{CDCl}_{3}\right) \delta 0.98(6 \mathrm{H}, \mathrm{d}, J=6.8 \mathrm{~Hz}), 2.22(1 \mathrm{H}, \mathrm{m}), 2.74(2 \mathrm{H}, \mathrm{d}, J$ = 6.8 Hz), $3.89(3 \mathrm{H}, \mathrm{s}), 7.32-7.39(1 \mathrm{H}, \mathrm{m}), 7.51-7.59(1 \mathrm{H}, \mathrm{m}), 7.60-7.67(1 \mathrm{H}$, m), 8.02-8.08 (1H, m); ${ }^{13} \mathrm{C} \mathrm{NMR}\left(\mathrm{CDCl}_{3}\right) \delta 22.6\left(\mathrm{CH}_{3}\right), 25.1(\mathrm{CH}), 50.0\left(\mathrm{CH}_{2}\right), 52.6\left(\mathrm{CH}_{3}\right), 85.8(\mathrm{C})$, $126.2(\mathrm{CH}), 127.6(\mathrm{C}), 130.0(\mathrm{CH}), 130.4(\mathrm{CH}), 132.7(\mathrm{CH}), 140.4(\mathrm{C}), 165.6(\mathrm{C}), 186.0(\mathrm{C}), 191.1(\mathrm{C})$; MS (EI) m/z $260\left(\mathrm{M}^{+}-28\right), 246,232,213,203,289,176,163,147,133,115,104,92,76,65,57,47,37$, 26.

8: Yellow solid; $\operatorname{mp} 35-37{ }^{\circ} \mathrm{C}$; IR (KBr) 3003, 2955, 2440, 2135, 1721, 1660, 1597, 1518,<smiles>CCCCCC(=O)C(=O)C(=O)c1ccccc1C(=O)OC</smiles>
1439, 1304, 1277, 1196, 1163, $1082 \mathrm{~cm}^{-1} ;{ }^{1} \mathrm{H} \mathrm{NMR}\left(\mathrm{CDCl}_{3}\right) \delta 0.90(3 \mathrm{H}, \mathrm{t}$, $J=7.1 \mathrm{~Hz}), 1.32(4 \mathrm{H}, \mathrm{m}), 1.67(2 \mathrm{H}$, quint, $J=7.3 \mathrm{~Hz}), 2.83(2 \mathrm{H}, \mathrm{t}, J=$ $7.8 \mathrm{~Hz}), 3.89(3 \mathrm{H}, \mathrm{s}), 7.34-7.37(1 \mathrm{H}, \mathrm{m}) 7.53-7.66(2 \mathrm{H}, \mathrm{m}), 8.03-8.05$ $(1 \mathrm{H}, \mathrm{m}) ;{ }^{13} \mathrm{C} \mathrm{NMR}\left(\mathrm{CDCl}_{3}\right) \delta 14.0\left(\mathrm{CH}_{3}\right), 22.5\left(\mathrm{CH}_{2}\right), 23.9\left(\mathrm{CH}_{2}\right), 31.3\left(\mathrm{CH}_{2}\right), 41.0\left(\mathrm{CH}_{2}\right), 52.6\left(\mathrm{CH}_{3}\right)$, $85.6(\mathrm{C}), 126.2(\mathrm{CH}), 127.6(\mathrm{C}), 129.9(\mathrm{CH}), 130.4(\mathrm{CH}), 132.7(\mathrm{CH}), 140.4(\mathrm{C}), 165.6(\mathrm{C}), 186.0(\mathrm{C})$, 191.7 (C); MS (EI) m/z 274 (M+28), 259, 243, 231, 218, 208, 200, 186, 175, 163, 147, 133, 115, 104, $92,77,63,50,39,26$.

9: Yellow viscous oil; IR (neat) 3021, 2934, 2134, 1717, 1651, 1557, 1539, 1507, 1456, 1435,<smiles>COC(=O)C(C(=O)c1ccccc1Cl)C1CCCCC1</smiles>
$1373,1287,1215,1059 \mathrm{~cm}^{-1} ;{ }^{1} \mathrm{H}$ NMR $\left(\mathrm{CDCl}_{3}\right) \delta 1.19-1.49(5 \mathrm{H}, \mathrm{m}), 1.68-$ $1.91(5 \mathrm{H}, \mathrm{m}), 3.21(1 \mathrm{H}, \mathrm{m}), 3.89(3 \mathrm{H}, \mathrm{s}), 7.34-7.37(1 \mathrm{H}, \mathrm{m}), 7.53-7.65(2 \mathrm{H}$, m), 8.03-8.05 (1H, m); ${ }^{13} \mathrm{C}$ NMR $\left(\mathrm{CDCl}_{3}\right) \delta 25.8\left(\mathrm{CH}_{2}\right), 25.9\left(\mathrm{CH}_{2}\right), 28.7$ 


\section{Supporting Information}

$\left(\mathrm{CH}_{2}\right), 47.9(\mathrm{CH}), 52.6\left(\mathrm{CH}_{3}\right), 85.0(\mathrm{C}), 126.3(\mathrm{CH}), 127.6(\mathrm{C}), 130.0(\mathrm{CH}), 130.4(\mathrm{CH}), 132.8(\mathrm{CH})$, 140.5 (C), 165.6 (C), 185.9 (C), 194.7 (C); MS (EI) m/z 287, 272, 258, 242, 226, 203, 197, 183, 173, 160, 146, 133, 120, 105, 92, 76, 64, 47, 35, 24, 13.

10: Pale yellow oil; IR (neat) 3025, 2955, 2175, 2127, 1722, 1659, 1597, 1578, 1495, 1455,<smiles>COC(=O)c1ccccc1C(=O)C(=O)Cc1ccccc1</smiles>
$1435,1392,1286,1217,1190,1140,1086,1032,1003,901,750,615 \mathrm{~cm}^{-1} ;{ }^{1} \mathrm{H}$ NMR $\left(\mathrm{CDCl}_{3}\right) \delta 3.81(3 \mathrm{H}, \mathrm{s}), 4.21(2 \mathrm{H}, \mathrm{s}), 7.24-8.09(9 \mathrm{H}, \mathrm{m}) ;{ }^{13} \mathrm{C} \mathrm{NMR}$ $\left(\mathrm{CDCl}_{3}\right) \delta 46.9\left(\mathrm{CH}_{2}\right), 52.6\left(\mathrm{CH}_{3}\right), 85.7(\mathrm{C}), 126.3(\mathrm{CH}), 127.0(\mathrm{CH}), 127.7$ (C), $128.4(\mathrm{CH}), 129.5(\mathrm{CH}), 130.0(\mathrm{CH}), 130.5(\mathrm{CH}), 132.7(\mathrm{CH}), 133.5(\mathrm{C}), 140.1(\mathrm{C}), 165.5(\mathrm{C})$, 185.8 (C), 189.1 (C); MS (EI) m/z $294\left(\mathrm{M}^{+}-28\right)$, 280, 262, 250, 233, 218, 203, 191, 175, 163, 147, 133, $117,103,91,76,50,35$.

11: Pale yellow oil; IR (neat) 3021, 2955, 2402, 2125, 1722, 1658, 1535, 1497, 1437, 1370,<smiles>COC(=O)c1ccccc1C(=O)C(=O)CCc1ccccc1</smiles>
$1287,1215,1140,1088,995,956,754,700,667 \mathrm{~cm}^{-1} ;{ }^{1} \mathrm{H}$ NMR $\left(\mathrm{CDCl}_{3}\right) \delta$ $3.00(2 \mathrm{H}, \mathrm{t}, J=7.3 \mathrm{~Hz}), 3.21(2 \mathrm{H}, \mathrm{t}, J=7.3 \mathrm{~Hz}), 3.87(3 \mathrm{H}, \mathrm{s}), 7.15-8.08(9 \mathrm{H}$, $\mathrm{m}) ;{ }^{13} \mathrm{C} \mathrm{NMR}\left(\mathrm{CDCl}_{3}\right) \delta 30.1\left(\mathrm{CH}_{2}\right), 42.6\left(\mathrm{CH}_{2}\right), 52.5\left(\mathrm{CH}_{3}\right), 85.6(\mathrm{C})$, $125.9(\mathrm{CH}), 126.2(\mathrm{CH}), 127.5(\mathrm{C}), 128.1(\mathrm{CH}), 128.3(\mathrm{CH}), 130.0(\mathrm{CH})$, $130.3(\mathrm{CH}), 132.7(\mathrm{CH}), 140.1(\mathrm{C}), 140.4(\mathrm{C}), 165.5$ (C), 185.7 (C), 190.7 (C); MS (EI) m/z 308 (M+ 28), 276, 231, 189, 163, 133, 91, 65, 35, 13. 
Influence of Ionic Radius on Diastereo- and Enantioselectivity in the Reaction of Diazoacetophenone 1 with Butyl Vinyl Ether Using (4S,5S)-Pybox-Ph 2 and Lanthanoid Triflates as Catalysts.

The influence of ionic radius of lanthanoid metals on enantio- and diastereoselectivity was investigated in the reaction of diazoacetophenone $\mathbf{1}$ with butyl vinyl ether (2b) utilizing $(4 S, 5 S)$-Pybox$\mathrm{Ph}_{2}$-lanthanoid triflate complexes under reflux in $\mathrm{CH}_{2} \mathrm{Cl}_{2}$ (Scheme 1, Table 1 and Figure 1).

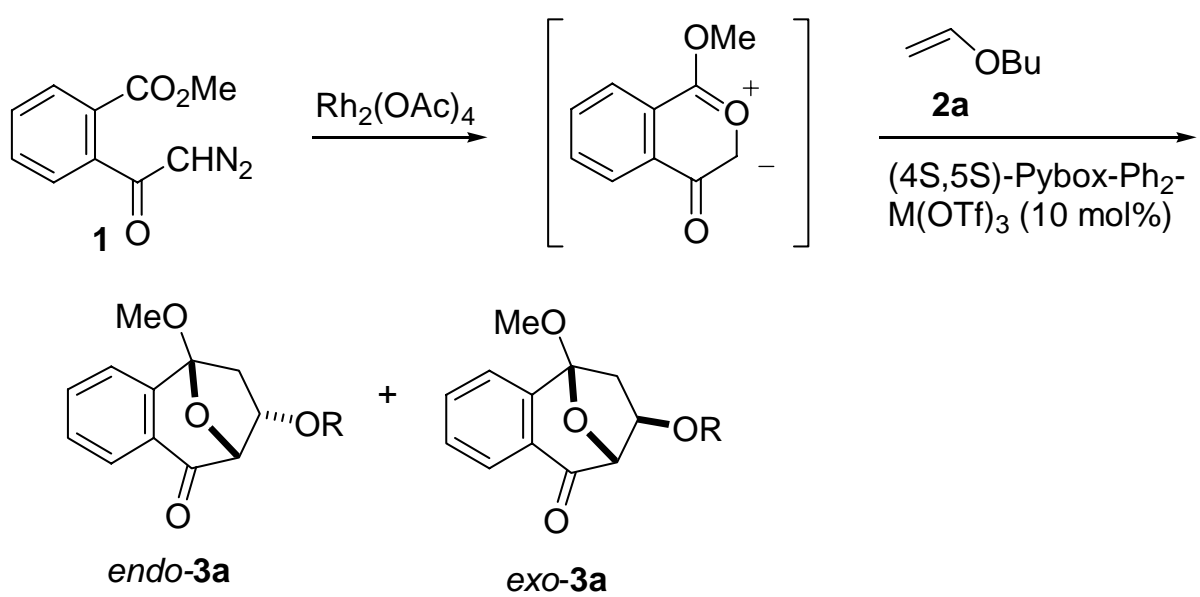

Scheme 1 


\section{Supporting Information}

Table 1. Reaction of Diazoacetophenone 1 with Butyl Vinyl Ether using (4S,5S)-Pybox- $\mathrm{Ph}_{2-}$ Lanthanoid Triflate Complexes ${ }^{\mathrm{a}}$

\begin{tabular}{|c|c|c|c|c|c|c|}
\hline \multirow{2}{*}{ entry } & \multirow{2}{*}{$\mathrm{M}(\mathrm{OTf})_{3}$} & \multirow{2}{*}{ ionic radius ${ }^{\mathrm{b}}$} & \multirow{2}{*}{ yield (\%) } & \multirow{2}{*}{ endo $: e x o^{\mathrm{c}}$} & \multicolumn{2}{|c|}{ ee $(\%)^{\mathrm{d}}$} \\
\hline & & & & & endo & exo \\
\hline 1 & $\mathrm{La}(\mathrm{OTf})_{3}$ & 1.032 & 87 & $76: 24$ & 51 & 34 \\
\hline 2 & $\mathrm{Sm}(\mathrm{OTf})_{3}$ & 0.958 & 74 & $79: 21$ & 59 & 44 \\
\hline 3 & $\mathrm{Eu}(\mathrm{OTf})_{3}$ & 0.947 & 93 & $81: 19$ & 81 & 56 \\
\hline 4 & $\mathrm{Gd}(\mathrm{OTf})_{3}$ & 0.938 & quant. & $81: 19$ & 85 & 62 \\
\hline 5 & $\mathrm{~Tb}(\mathrm{OTf})_{3}$ & 0.923 & 65 & $79: 21$ & 67 & 31 \\
\hline 6 & $\operatorname{Ho}(\mathrm{OTf})_{3}$ & 0.901 & 89 & $79: 21$ & 85 & 66 \\
\hline 7 & $\operatorname{Er}(\mathrm{OTf})_{3}$ & 0.890 & 97 & $79: 21$ & 84 & 71 \\
\hline 8 & $\operatorname{Tm}(\mathrm{OTf})_{3}$ & 0.880 & 78 & $77: 23$ & 81 & 52 \\
\hline 9 & $\mathrm{Yb}(\mathrm{OTf})_{3}$ & 0.868 & 80 & $75: 25$ & 67 & 40 \\
\hline
\end{tabular}

${ }^{\text {a }}$ The reaction was carried out by adding a solution of $\mathbf{1}$ in $\mathrm{CH}_{2} \mathrm{Cl}_{2}$ to a suspension of $\mathbf{2 a}-\mathbf{d}$,

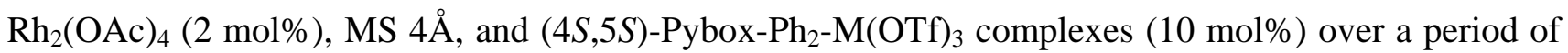
$1 \mathrm{~h} .{ }^{\mathrm{b}}$ See ref. 5. ${ }^{\mathrm{c}}$ Determined by ${ }^{1} \mathrm{H}$ NMR. ${ }^{\mathrm{d}}$ Enantiomeric excess of endo-adduct determined by chiral HPLC. 


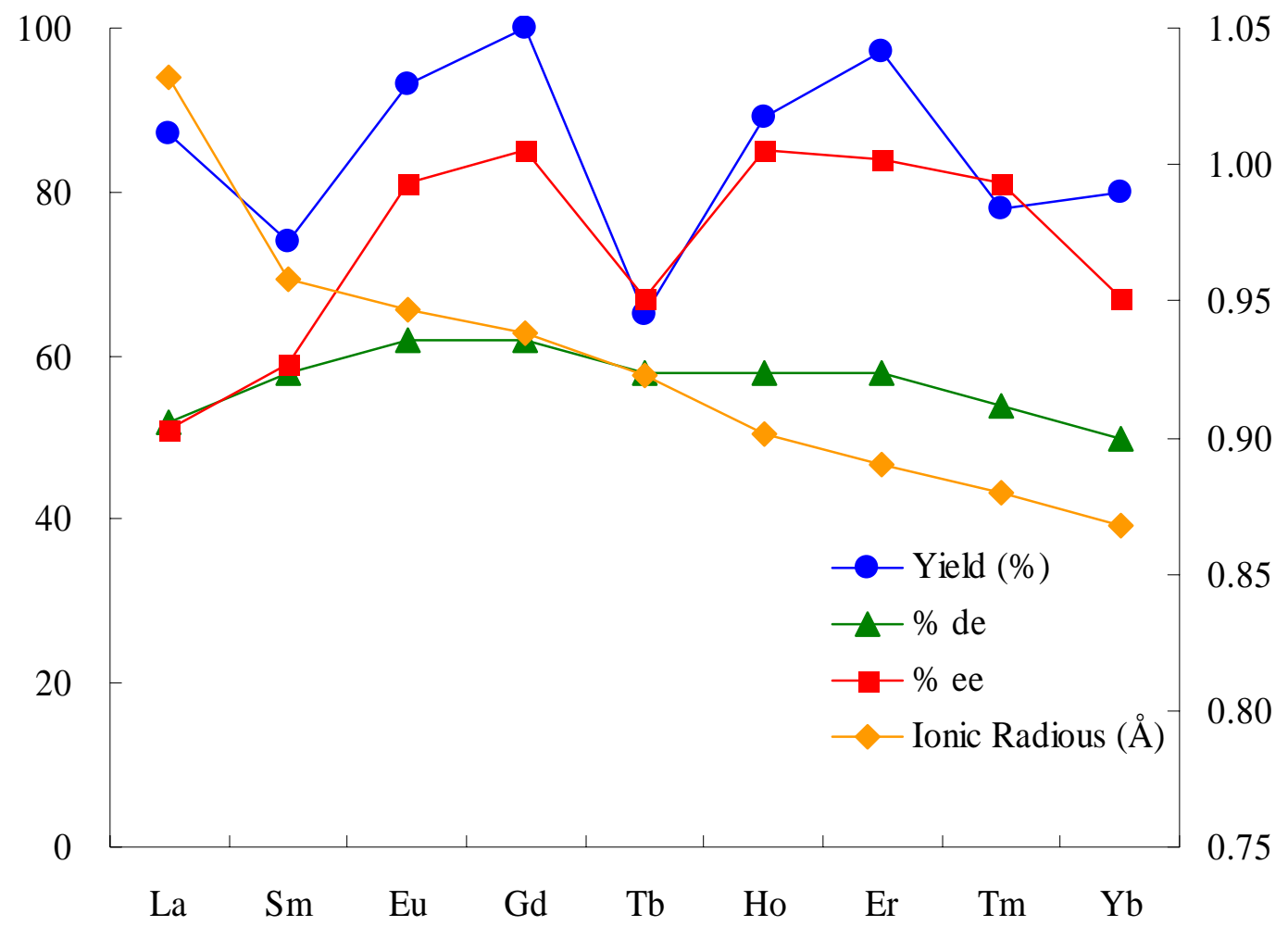

Figure 1. Relationship between metal and enantio- and diastereoselectivity in (4S,5S)-Pybox$\mathrm{Ph}_{2}-\mathrm{M}(\mathrm{OTf})_{3}$ catalyzed reaction of Diazoacetophenone 1 with Butyl Vinyl Ether. 


\section{Supporting Information}

\section{The interaction between HOMO and LUMO on the basis of frontier orbital theory.}

The energy levels of the HOMO and the LUMO of carbonyl ylides (A and $\mathbf{B}$ ) generated from diazo compounds $\mathbf{1}$ and $\mathbf{4}$, respectively, and cylcohexyl vinyl ether (2d) were calculated by semiempirical molecular orbital calculations (PM3) using Spartan '04 (Figure 1). The calculations indicated that the strongest interaction is between the LUMO of the carbonyl ylides and the HOMO of cyclohexyl vinyl ether (2d).<smiles>COC1=[O+]C(C)C(=O)c2ccccc21</smiles>

A<smiles>C=COC1CCCCC1</smiles>

LUMO (1.37)

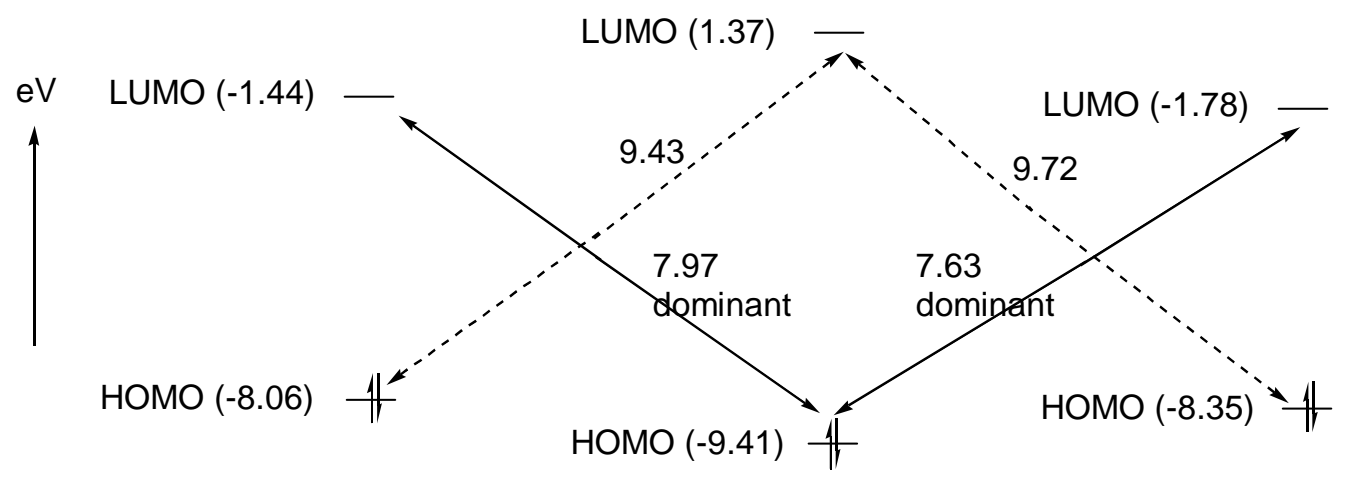

Figure 2. The interaction between HOMO and LUMO.

\section{Reaction of epoxy compound I as a carbonyl ylide precursor.}

Epoxy compound I was prepared from diazo compound $\mathbf{7}$ according to the procedure reported by Padwa. ${ }^{2}$ Epoxide I was obtained only as a $97: 3$ (I : II) mixture with compound II after florisil (100 - 200 mesh) flash chromatography, because epoxide I was easily hydrolyzed by chromatographic purification (Scheme 2). The mixture of epoxide I (97 : 3 (I/II)) and cyclohexyl vinyl ether (2 equiv) was treated in the presence of $(R)$-BINIM-4Me-2QN-Ni(II) catalyst (10 mol\%) under 


\section{Supporting Information}

reflux in $\mathrm{CH}_{2} \mathrm{Cl}_{2}$ or $\mathrm{CHCl}_{3}$ for $22 \mathrm{~h}$ or $3 \mathrm{~h}$, respectively (Scheme 3). Endo-cycloadduct (endo : exo = > 99 : 1) was obtained along with by-product II (trace and $9 \%$ yield, respectively) in $61 \%$ or $44 \%$, respectively. However, the enantiomeric excess of the endo-adduct was only $9 \%$ ee or $7 \%$ ee, respectively. Based on Padwa's results, ${ }^{2}$ much higher temperature $\left(100{ }^{\circ} \mathrm{C}\right)$ is probably required for smooth ring-opening of epoxide I to the corresponding carbonyl ylide. Considering from easy hydrolyzed property of epoxide $\mathbf{I}$, the reaction of epoxide I with cyclohexyl vinyl ether under above conditions might occur by stepwise mechanism starting from a nucleophilic attack of the vinily ether to the epoxide which was activated by a Lewis acid.<smiles>COC(=O)C(=NC(=O)c1ccccc1Cl)C(=O)CC(C)C</smiles>

7<smiles>COC12OC(C(=O)CC(C)C)(OC1=O)c1ccccc12</smiles>

I

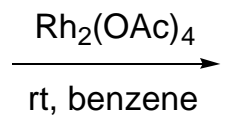<smiles>C=COC1CCCCC1</smiles>

(2 equiv)<smiles>COC12OC(C(=O)CC(C)C)(OC1=O)c1ccccc12</smiles>

I<smiles>CC(C)CC(=O)C1OC(=O)c2ccccc2C1=O</smiles>

II

Scheme 2

(R)-BINIM-4Me-2QN-Ni(II)

(10 mol\%)

reflux in $\mathrm{CH}_{2} \mathrm{Cl}_{2}$ or $\mathrm{CHCl}_{3}$<smiles>COC12C[C@H](OC3CCCCC3)[C@](C(=O)CC(C)C)(O1)C(=O)c1ccccc12</smiles>

$15 d$

Scheme 3 


\section{Supporting Information}

\section{References and Notes}

1) Ueda, K.; Ibata, T.; Takebayashi, M. Bull. Chem. Soc. Jpn. 1972, 45, 2779.

2) Padwa, A.; Boonsombat, J.; Rashatasakhon, P.; Willis, J. Org. Lett. 2005, 7, 3725.

3) (S,S)-Pybox-i-Pr and (4S,5S)-Pybox-Ph 2 : (a) Totleben, M. J.; Prasad, J. S.; Simpson, J. H.; Chan, S. H.; Vanyo, D. J.; Kuehner, D. E.; Deshpande, R.; Kodersha, G. A. J. Org. Chem. 2001, 66, 1057. (b) Desimoni, G.; Faita, G.; Filippone, S.; Mella, M.; Zampori, M. G.; Zema, M. Tetrahedron, 2001, 57, 10203. (S,S)-Pybox-Ph: (c) Davies, I. W.; Gerena, L.; Lu, N.; Larsen, R. D.; Reider, P. J. J. Org. Chem. 1996, 61, 9629.

4) (a) Suga, H.; Kakehi, A.; Mitsuda, M. Bull. Chem. Soc. Jpn. 2004, 77, 561. (b) Suga, H.; Kitamura, T.; Kakehi, A.; Baba, T. Chem. Commun. 2004, 1414. (c) Suga, H.; Nakajima, T.; Itoh, K.; Kakehi, A. Org. Lett. 2005, 7, 1431. (d) Suga, H.; Funyu, A. Kakehi, A. Org. Lett. 2007, 9, 97.

5) Shannon, R. D. Acta Crystallogr. 1976, A32, 751.

6) Padwa, A.; Fryxell, G. E.; Zhi, L. J. Am. Chem. Soc. 1990, 112, 3100.

7) (a) Suga, H.; Ebiura, Y.; Fukushima, K.; Kakehi, A.; Baba, T. J. Org. Chem. 2005, 26, 10782. (b) Suga, H.; Inoue, K.; Inoue, S.; Kakehi, A. J. Am. Chem. Soc. 2002, 124, 14836. (c) Suga, H.; Inoue, K.; Inoue, S.; Kakehi, A.; Shiro, M. J. Org. Chem. 2005, 70, 47. 


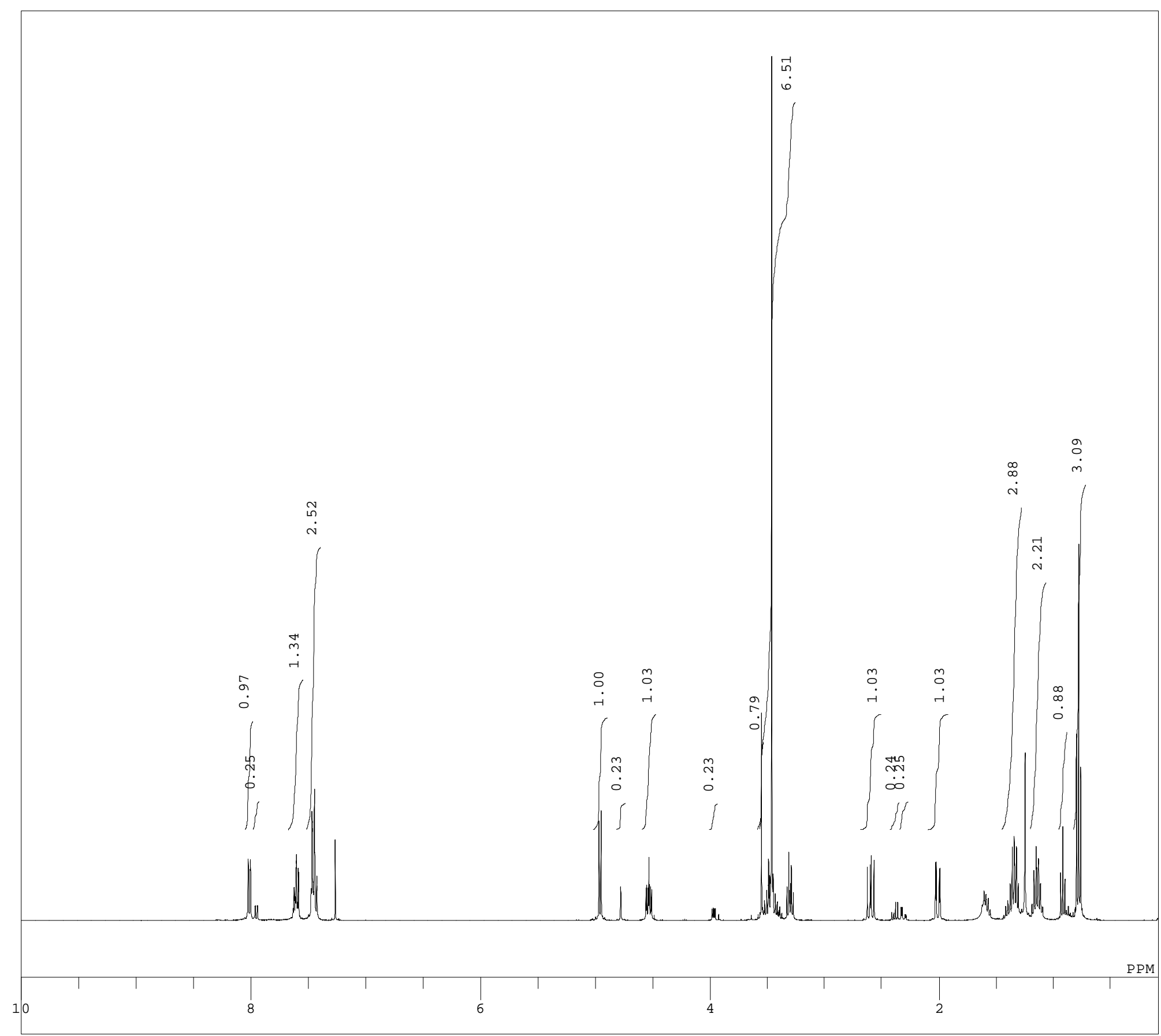

DFILE $\quad C: \backslash$ Documents and Settings $\backslash H$ Hro $\backslash f f f X f N f g f b f V$ COMNT DI

DATIM Thu Oct O5 15:12:23 200

OBNUC $1 \mathrm{H}$

EXMOD NON

OBFRQ $\quad 399.65 \mathrm{MHZ}$

OBSET $\quad 124.00 \mathrm{KHZ}$

OBFIN $\quad 10500.0 \mathrm{HZ}$

$\begin{array}{lr}\text { POINT } & 32768 \\ \text { FREQU } & 8000.0 \mathrm{~Hz}\end{array}$

$\begin{array}{lc}\text { FREQU } & 8000.0 \mathrm{~Hz} \\ \text { SCANS } & 8\end{array}$

$\begin{array}{lr}\text { SCANS } & 8 \\ \text { ACQTM } & 4.096 \mathrm{sec}\end{array}$

$\mathrm{PD} \quad 4.950 \mathrm{sec}$

10.0 us

$\begin{array}{lll}\text { IRNUC } & \text { IH } & \\ \text { CTEMP } & & 25.8 \text { C }\end{array}$

SLVNT CDCL3

SLVNT

$25.8 \mathrm{C}$

EXRE

$0.00 \mathrm{ppm}$
$0.24 \mathrm{~Hz}$
$18 \mathrm{~m}$ .24
18

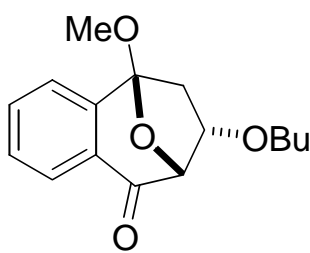

endo $:$ exo $=81: 19$ 


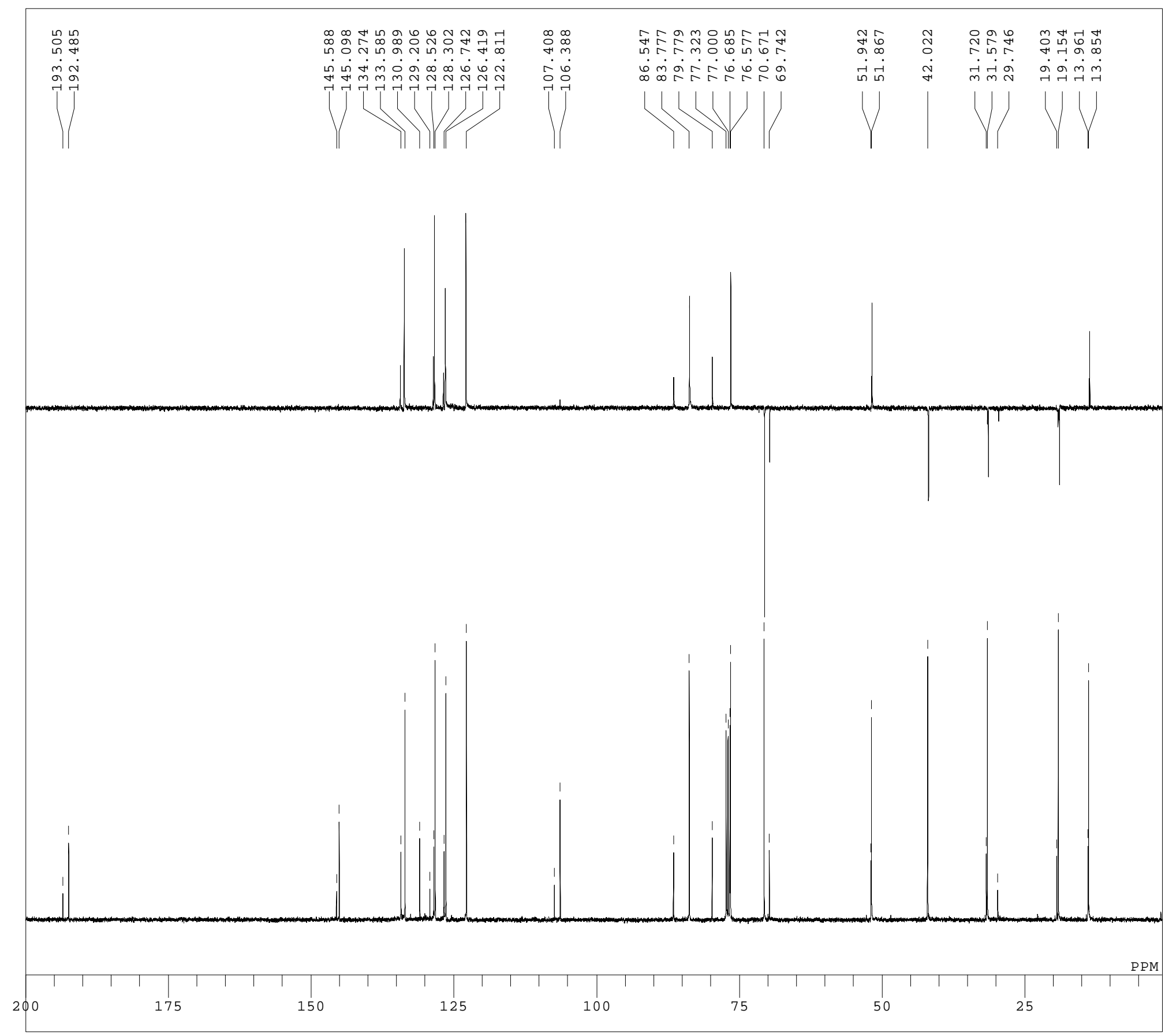

DFILE C: $\backslash$ Documents and Settings $\backslash H i r o \backslash f f f X f N f g f b f v$ COMNT DI-251-1DEPT

DATIM Sun Feb 25 11:31:20 200

OBNUC 13C

$\begin{array}{lll}\text { EXMOD } & \text { BCM } \\ \text { OBFRQ } & 100.40 \mathrm{MH}\end{array}$

OBSET $\quad 125.00 \mathrm{KHz}$

OBFIN $\quad 10500.0 \mathrm{~Hz}$

$\begin{array}{lr}\text { POINT } & 32768 \\ \text { FREQU } & 27322.4\end{array}$

SCANS $\quad 800$

ACQTM $\quad 1.199 \mathrm{sec}$

$\begin{array}{lr}\text { PD } & 3.000 \mathrm{sec} \\ \text { PW1 } & 6.3 \mathrm{us}\end{array}$

IRNUC $1 \mathrm{H} \quad 6.3$ us

CTEMP 25.9

SLVNT CDCL3

EXREF

$77.00 \mathrm{ppm}$

1.20

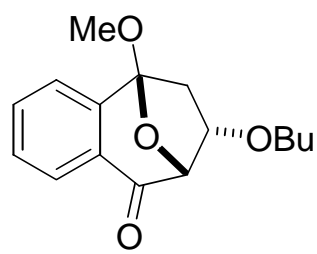

endo $:$ exo $=81: 19$ 


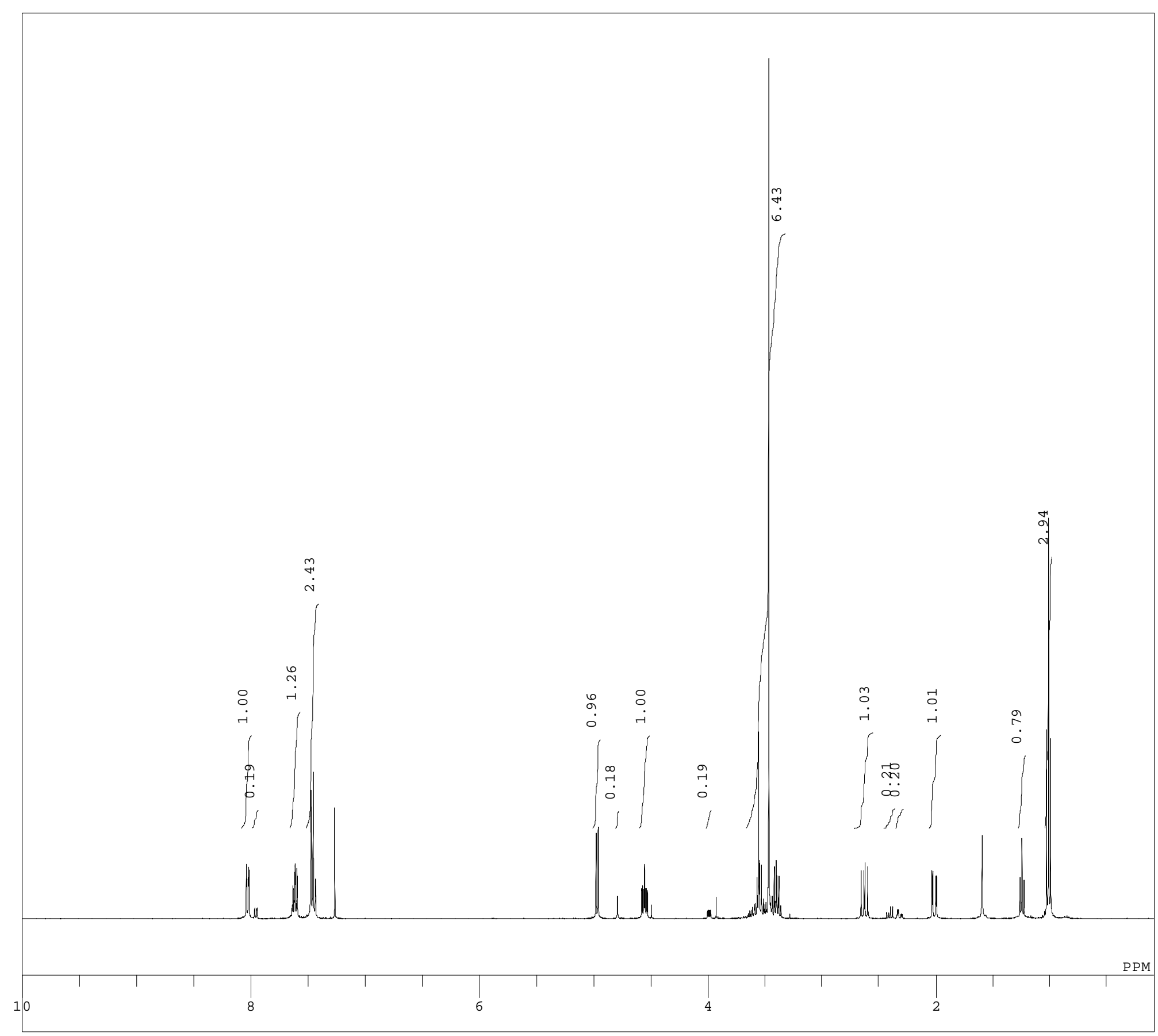

DFILE C: $\backslash$ Documents and Settings $\backslash H i r o \backslash f f f X f N f g f b f v$

COMNT DI

DATIM Tue Nov 14 01:31:46 200

OBNUC $1 \mathrm{H}$

EXMOD NON

OBFRQ $\quad 399.65 \mathrm{MHZ}$

OBSET $\quad 124.00 \mathrm{KHZ}$

OBFIN $\quad 10500.0 \mathrm{~Hz}$

POINT 32768

$\quad 8000.0 \mathrm{~Hz}$

SCANS

$\mathrm{ACQT}$

$\mathrm{PD}$

$4.096 \mathrm{sec}$

$4.950 \mathrm{sec}$

10.0 us

CTEMP 20.8

SLVNT CDCL3

EXREF

$0.00 \mathrm{ppm}$

$0.12 \mathrm{~Hz}$

$\mathrm{MeO}$

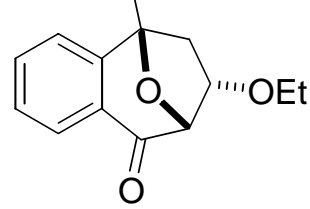

endo $:$ exo $=83: 17$ 


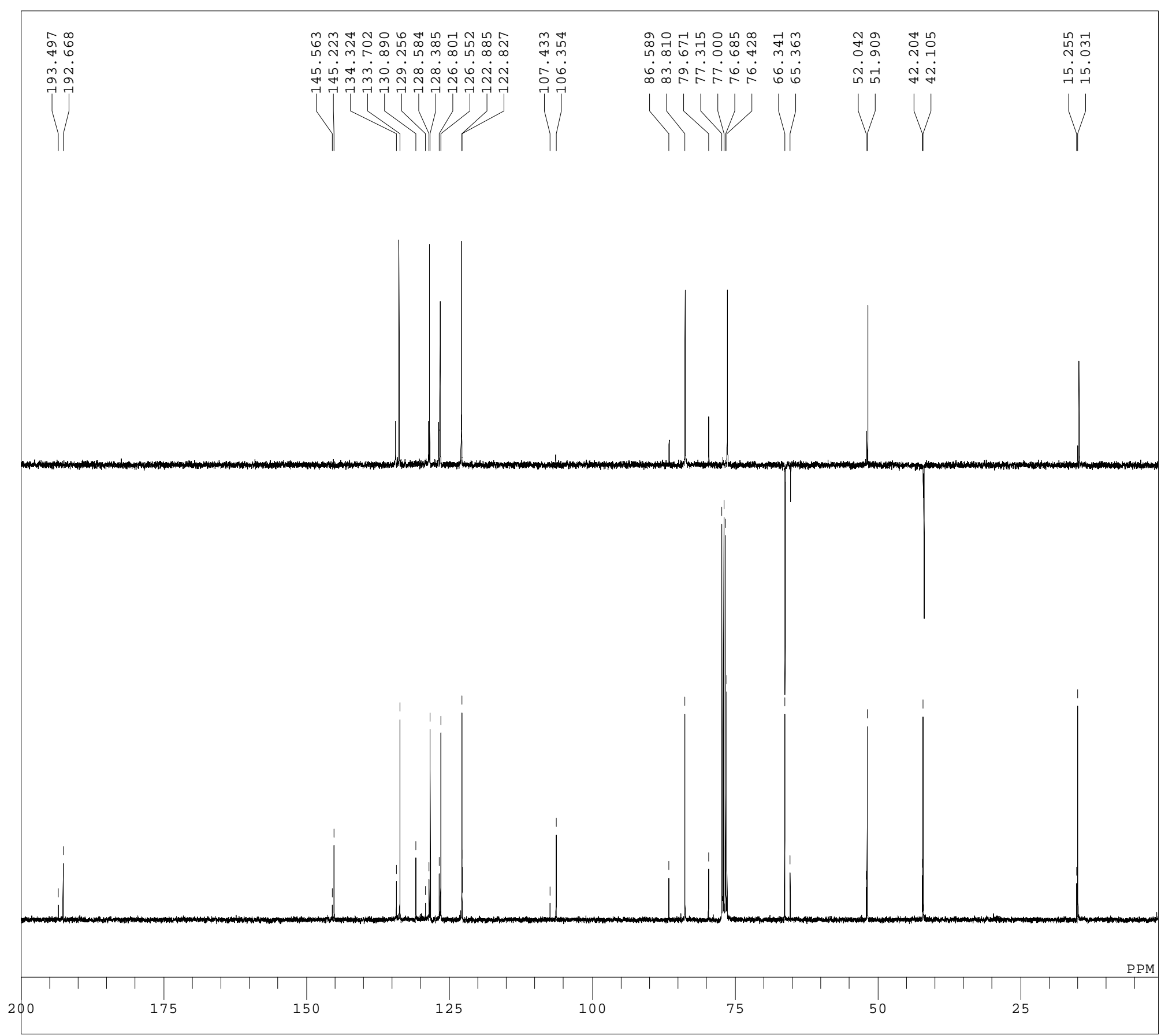

DFILE $\quad C: \backslash$ Documents and Settings $\backslash$ Hiro $\backslash f f f X f N f g f b f v$ COMNT DI

DATIM Thu Feb 22 04:53:42 200

OBNUC $13 \mathrm{C}$

EXMOD BCM

$\begin{array}{ll}\text { OBFRQ } & 100.40 \mathrm{MHz}\end{array}$

$\begin{array}{ll}\text { OBSET } & 125.00 \mathrm{KHZ}\end{array}$

OBFIN $\quad 10500.0 \mathrm{~Hz}$

$\begin{array}{rr}\text { POINT } & 32768 \\ \text { FREQU } & 27322.4\end{array}$

$\begin{array}{rr}\text { FREQU } & 27322.4 \\ \text { SCANS } & 1600\end{array}$

ACQTM $\quad 1.199 \mathrm{sec}$

PD $\quad 3.000 \mathrm{sec}$

$\begin{array}{ll}\text { PW1 } & 6.3 \text { us }\end{array}$

$\begin{array}{lll}\text { IRNUC } & 1 \mathrm{H} & 25.3 \mathrm{C} \\ \text { CTEMP } & & \end{array}$

SLVNT CDCL3

$\begin{array}{lll}\text { EXREF } & 77.00 \mathrm{ppm}\end{array}$

$\begin{array}{rl}77.00 & \mathrm{ppm} \\ 1.20 \mathrm{~Hz} & \end{array}$

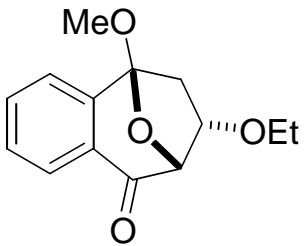

endo $:$ exo $=83: 17$ 


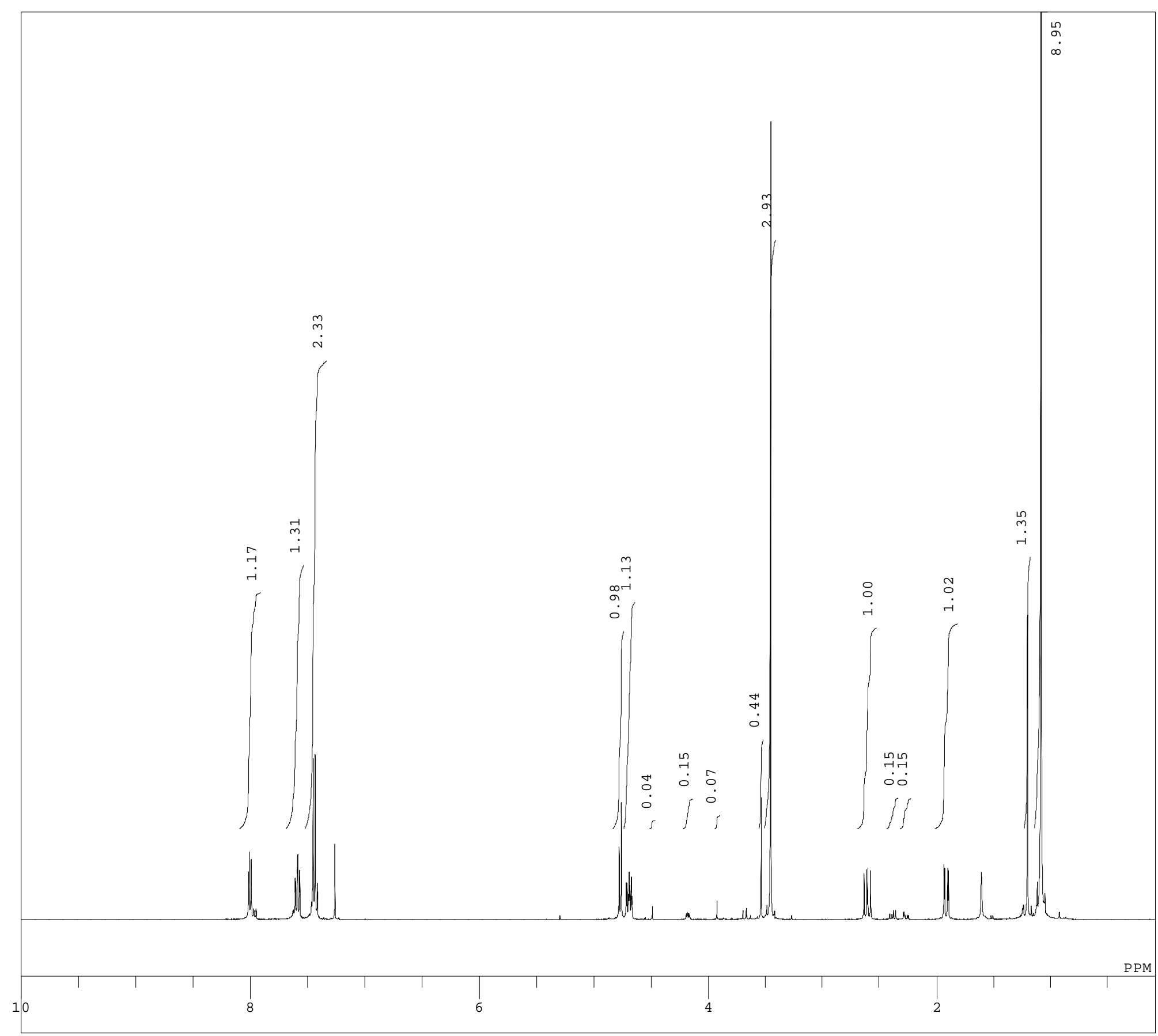

DFILE $\quad C: \backslash$ Documents and Settings $\backslash H i r o \backslash f f f X f N f g f b f v \backslash$

COMNT DI

DATIM Mon Oct 23 21:02:23 200

OBNUC $1 \mathrm{H}$

EXMOD NON

OBFRQ $\quad 399.65 \mathrm{MHz}$

OBSET $\quad 124.00 \mathrm{KHZ}$

OBFIN $\quad 10500.0 \mathrm{~Hz}$

POINT 32768

FREQU $7993.6 \mathrm{~Hz}$

$\begin{array}{lr}\text { SCANS } & 16 \\ \text { ACQTM } & 4.099 \mathrm{sec}\end{array}$

$\begin{array}{ll}\text { ACQTM } & 4.099 \mathrm{sec} \\ \mathrm{PD} & 4.950 \mathrm{sec}\end{array}$

PW1 10.0 us

IRNUC 1H

SLVNT CDCL3

EXREF

$21.9 \mathrm{c}$

$0.00 \mathrm{ppm}$ .24

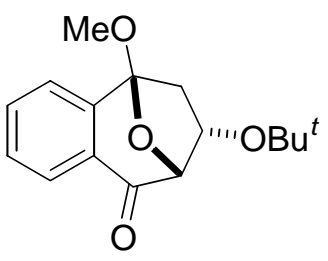

endo $:$ exo $=87: 13$ 


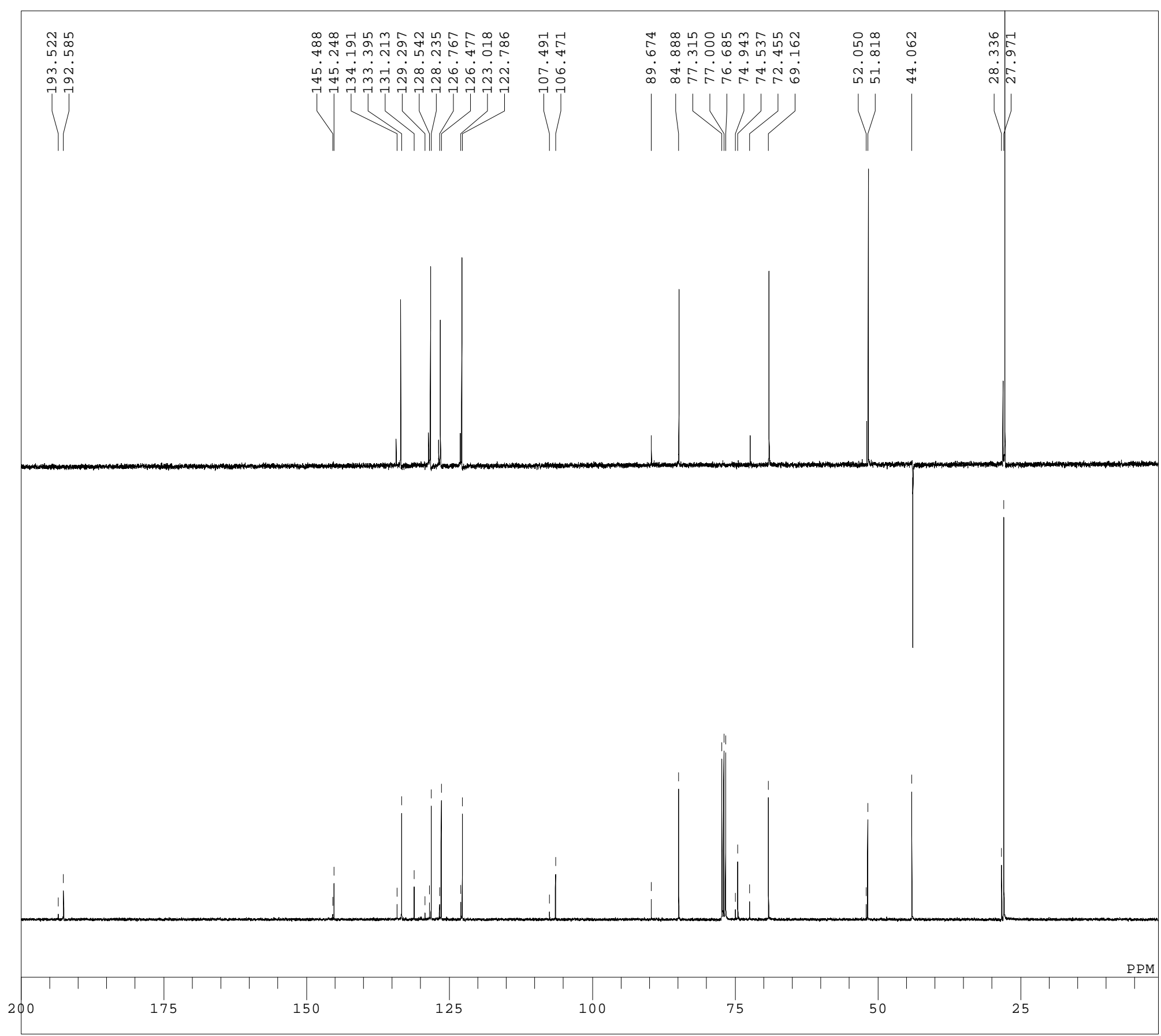

DFILE C: \Documents and Settings $\backslash H i r o \backslash f f f X f N f g f b f v$ COMNT DI-203-1DEPT

DATIM Mon Feb 19 21:03:13 200

OBNUC $13 \mathrm{C}$

$\begin{array}{lll}\text { EXMOD } & \text { BCM } & \\ \text { OBFRQ } & & 100.40 \mathrm{MHZ}\end{array}$

$\begin{array}{ll}\text { OBFRQ } & 100.40 \mathrm{MHz} \\ \text { OBSET } & 125.00 \mathrm{KHz}\end{array}$

OBFIN $\quad 10500.0 \mathrm{~Hz}$

POINT 32768

FREQU 27322.4

SCANS 1200

ACQTM $\quad 1.199 \mathrm{sec}$

PD $\quad 3.000 \mathrm{sec}$

PRN $1 \mathrm{H} \quad 6.3$ us

IRNUC $1 \mathrm{H}$

SLVNT CDCL3

EXREF

$27.1 \mathrm{C}$

$77.00 \mathrm{ppm}$

$.20 \mathrm{~Hz}$

\section{$\mathrm{MeO}$}

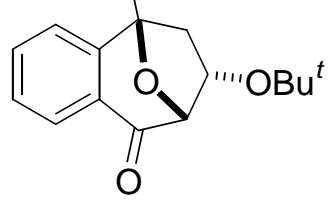

endo $:$ exo $=87: 13$ 


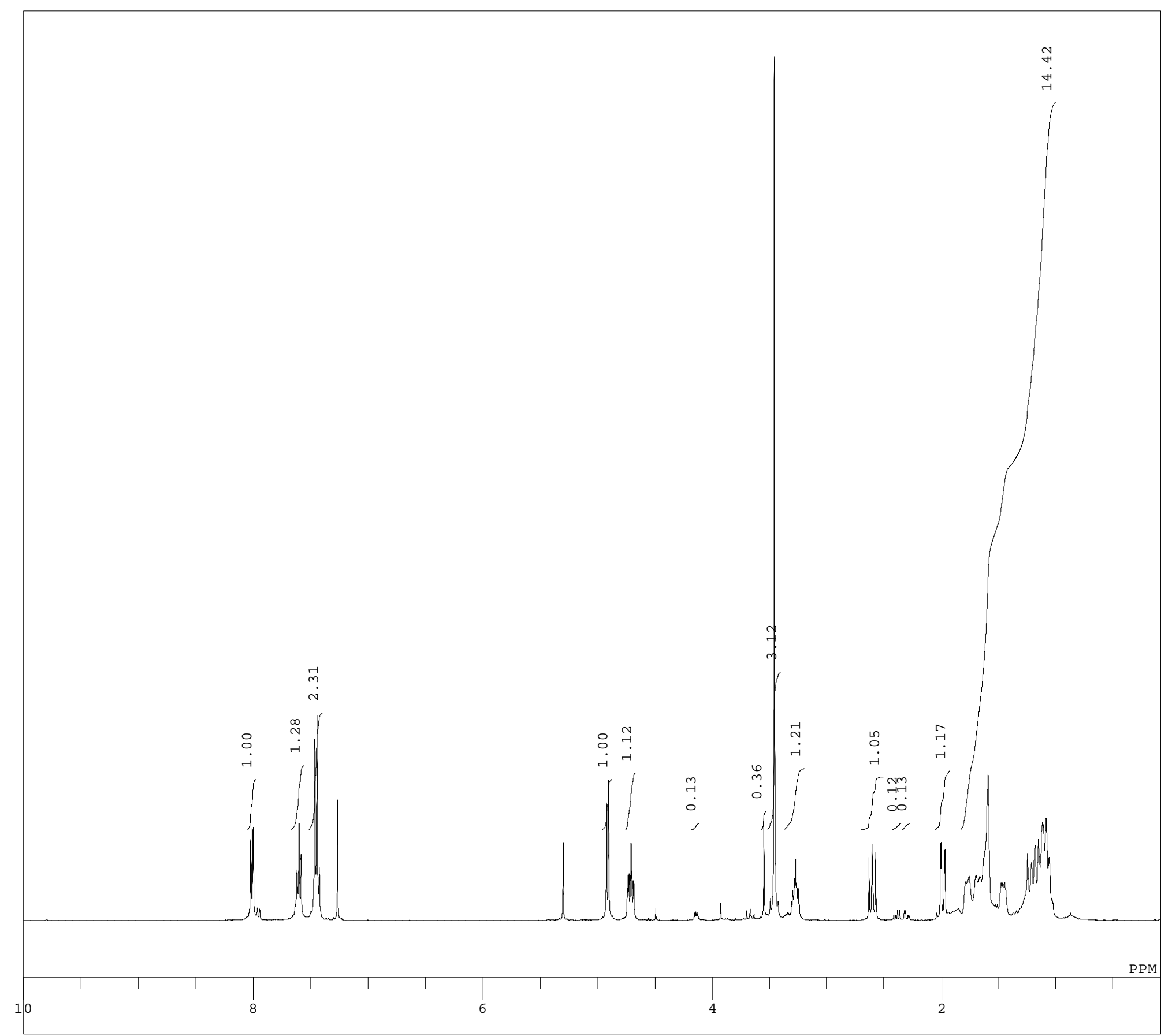

DFILE $\quad C: \backslash$ Documents and Settings $\backslash H$ Hro $\backslash f f f X f N f g f b f \mathrm{~V}$ COMNT DI

DATIM Thu Oct 26 19:48:40 2006

OBNUC $1 \mathrm{H}$

EXMOD NON

OBFRQ $\quad 399.65 \mathrm{MHZ}$

OBSET $\quad 124.00 \mathrm{KHZ}$

OBFIN $\quad 10500.0 \mathrm{~Hz}$

$\begin{array}{lr}\text { POINT } & 65536 \\ \text { FREQU } & 8000.0\end{array}$

$\begin{array}{cc}\text { FREQU } & 8000.0 \mathrm{~Hz} \\ \text { SCANS } & 8\end{array}$

$\begin{array}{lr}\text { SCANS } & 8 \\ \text { ACQTM } & 4.096 \mathrm{sec}\end{array}$

PD $\quad 4.950 \mathrm{sec}$

PW1 10.0 us

IRNUC $1 \mathrm{H}$

SLVNT CDCL3

EXREF

$20.9 \mathrm{c}$

$0.00 \mathrm{ppm}$

$.20 \mathrm{H}$

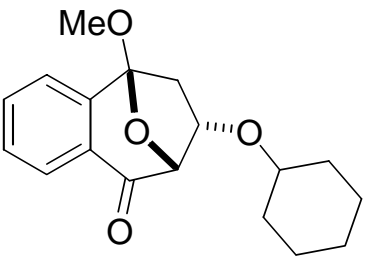

endo $:$ exo $=88: 12$ 


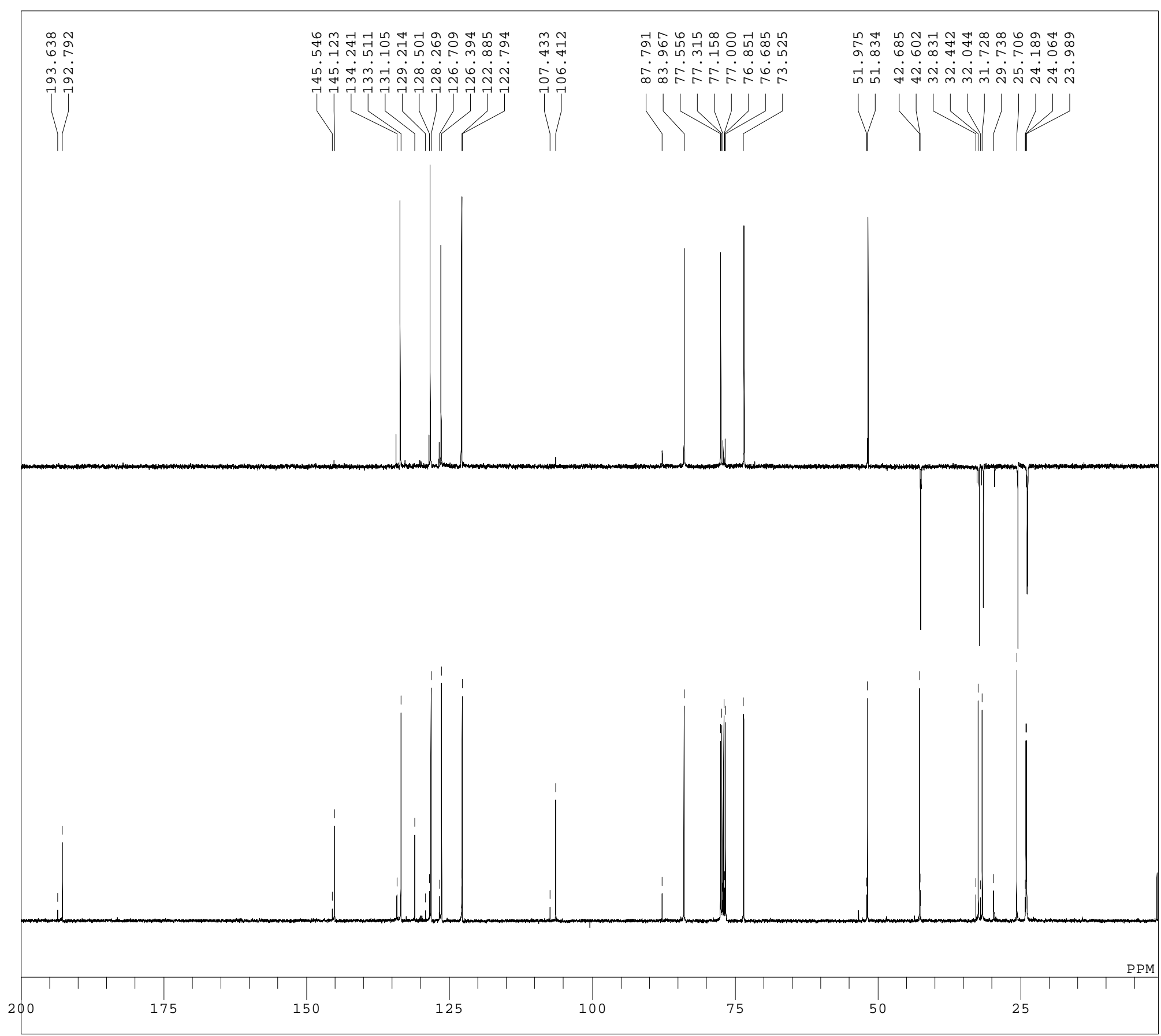

DFILE $\quad C: \backslash$ Documents and Settings $\backslash H i r o \backslash f f f X f N f g f b f v \backslash$ COMNT DI-208-1DEPT

DATIM Sun Feb $18 \quad 20: 44: 32 \quad 2007$

OBNUC $13 \mathrm{C}$

$\begin{array}{lll}\text { EXMOD } & \text { BCM } & \\ \text { OBFRQ } & & 100.40 \mathrm{MHZ}\end{array}$

$\begin{array}{ll}\text { OBFRQ } & 100.40 \mathrm{MHz} \\ \text { OBSET } & 125.00 \mathrm{KHz}\end{array}$

OBFIN $\quad 10500.0 \mathrm{~Hz}$

POINT 32768

FREQU $\quad 27322.4 \mathrm{~Hz}$

SCANS 1200

ACQTM $\quad 1.199 \mathrm{sec}$

PD

$3.000 \mathrm{sec}$
6.3 us

IRNUC $1 \mathrm{H}$

CTEMP

SLVNT

25.3

EXREF

$77.00 \mathrm{ppm}$

$1.20 \mathrm{~Hz}$
31

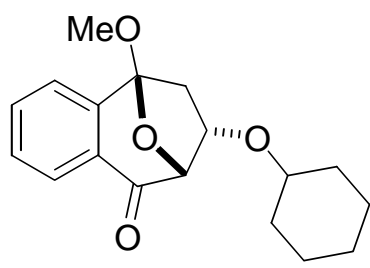

endo $:$ exo $=88: 12$ 


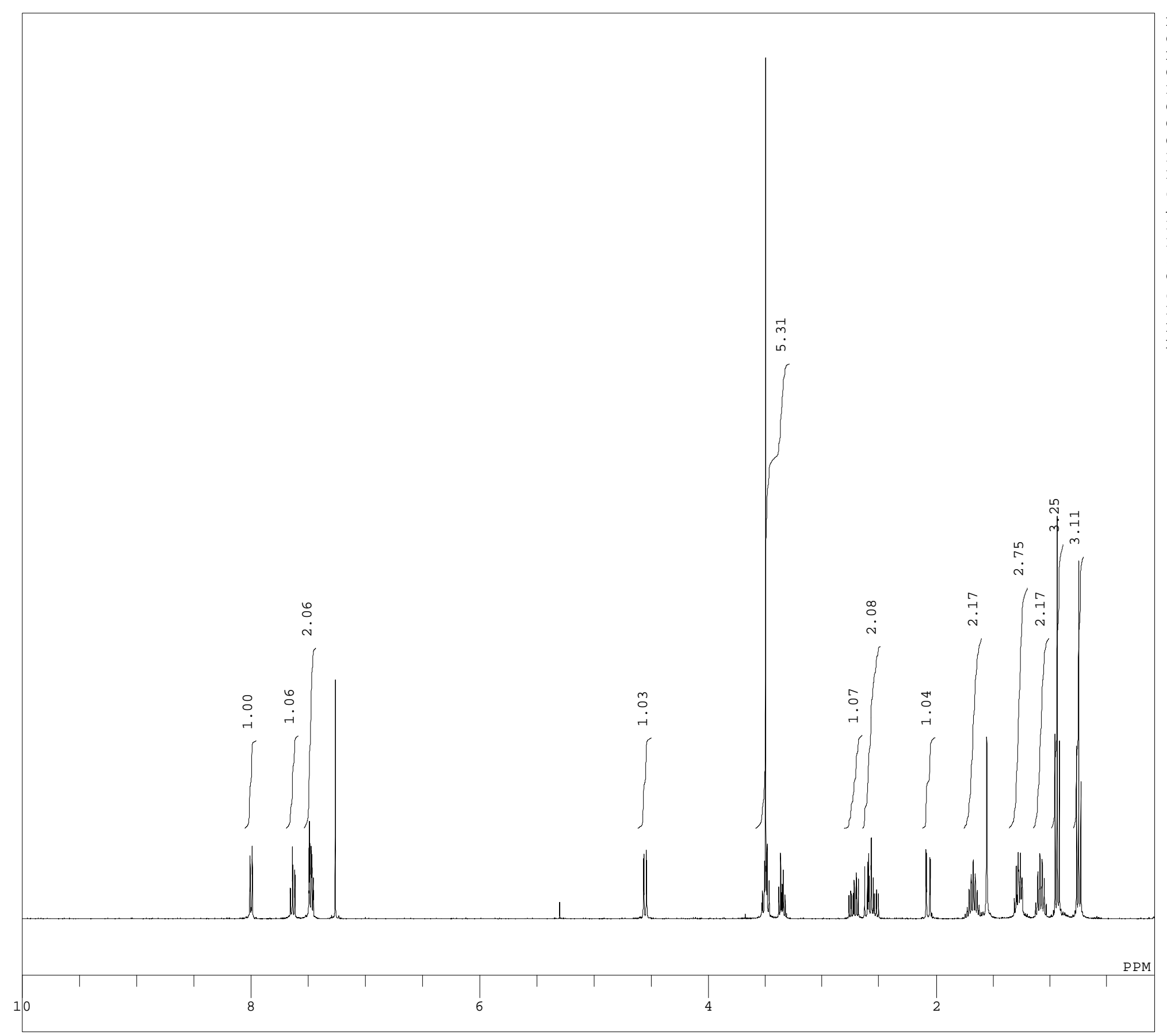

DFILE C: $\backslash$ Documents and Settings $\backslash H i r o \backslash f f f X f N f g f b f v$ COMNT DI

DATIM Sat Dec 10 23:12:05 2005

OBNUC $1 \mathrm{H}$

EXMOD NON

OBFRQ $\quad 399.65 \mathrm{MHZ}$

OBSET $\quad 124.00 \mathrm{KHZ}$

OBFIN $\quad 10500.0 \mathrm{~Hz}$

POINT 32768

$\quad 8000.0$

SCANS 16

ACQTM $\quad 4.096 \mathrm{sec}$

PD $\quad 4.950 \mathrm{sec}$

IRNUC $1 \mathrm{H} 10.0$ us

IRNUC 1

SLVNT CDCL3

EXREF

$24.1 \mathrm{C}$

$0.00 \mathrm{ppm}$

$.12 \mathrm{~Hz}$

$\mathrm{MeO}$

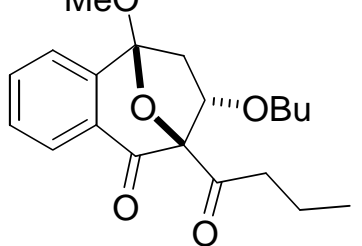




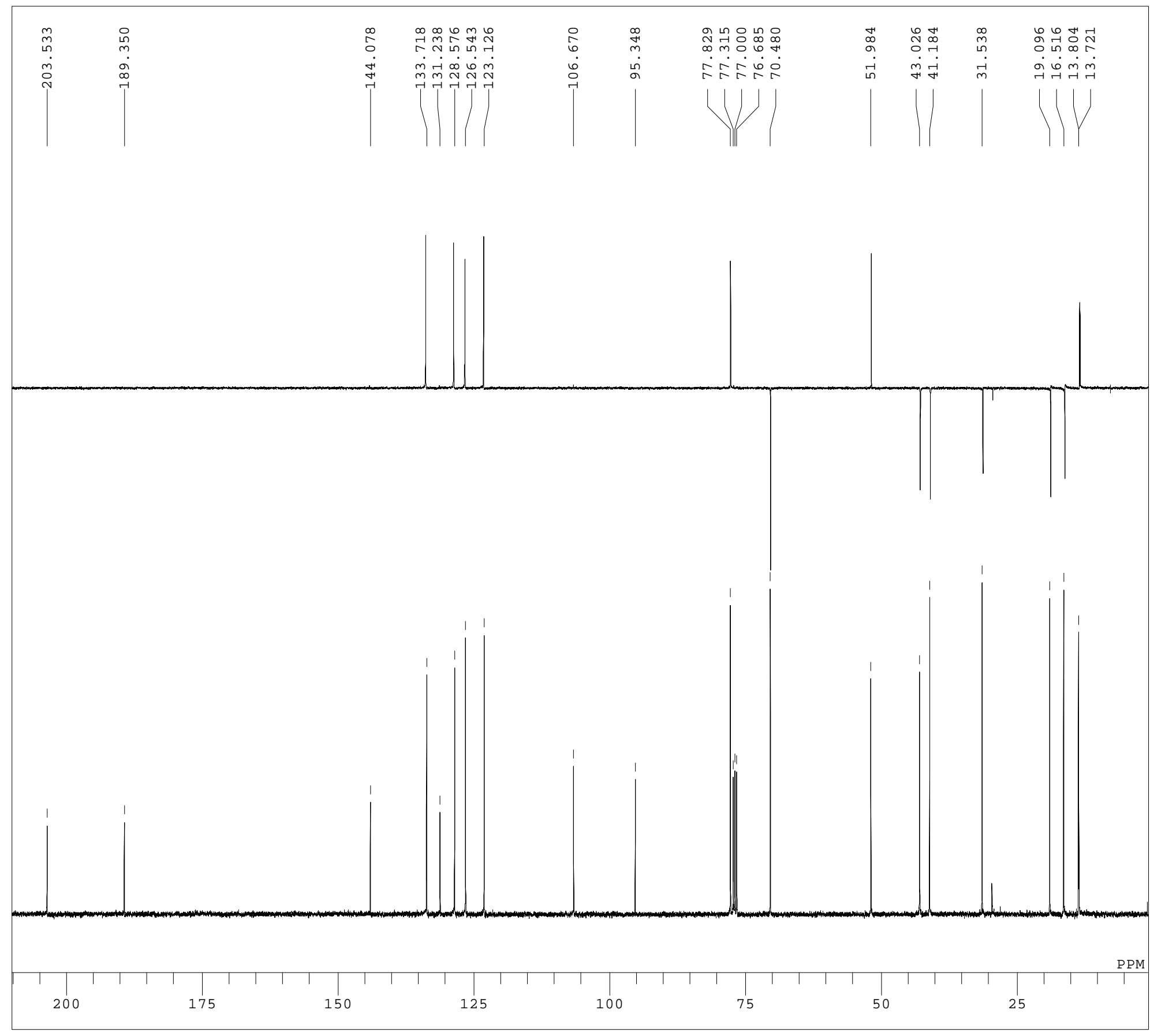

DFILE C: $\backslash$ Documents and Settings $\backslash H i r O \backslash f f f X f N f g f b f v \backslash$ COMNT DI

DATIM Fri Jan 26 22:42:22 2007

OBNUC $13 \mathrm{C}$

EXMOD BCM

OBFRQ $\quad 100.40 \mathrm{MHz}$

$\begin{array}{ll}\text { OBSET } & 125.00 \mathrm{KHZ}\end{array}$

OBFIN $\quad 10500.0 \mathrm{~Hz}$

$\begin{array}{lr}\text { POINT } & 32768 \\ \text { FREQU } & 27322.4\end{array}$

FREQU 27322.4

SCANS 200

ACQTM $\quad 1.199 \mathrm{sec}$

$\begin{array}{lr}\text { PD } & 3.000 \mathrm{sec} \\ \text { PW1 } & 6.3 \mathrm{us}\end{array}$

IRNUC $1 \mathrm{H}$

CTEMP 25.4

SLVNT CDCL3

$\begin{array}{ll}\text { EXREF } & 77.00 \mathrm{ppm}\end{array}$

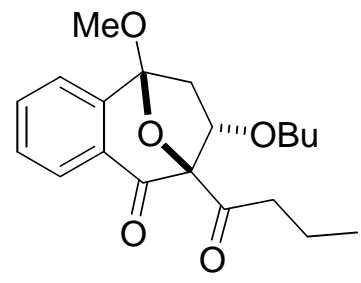




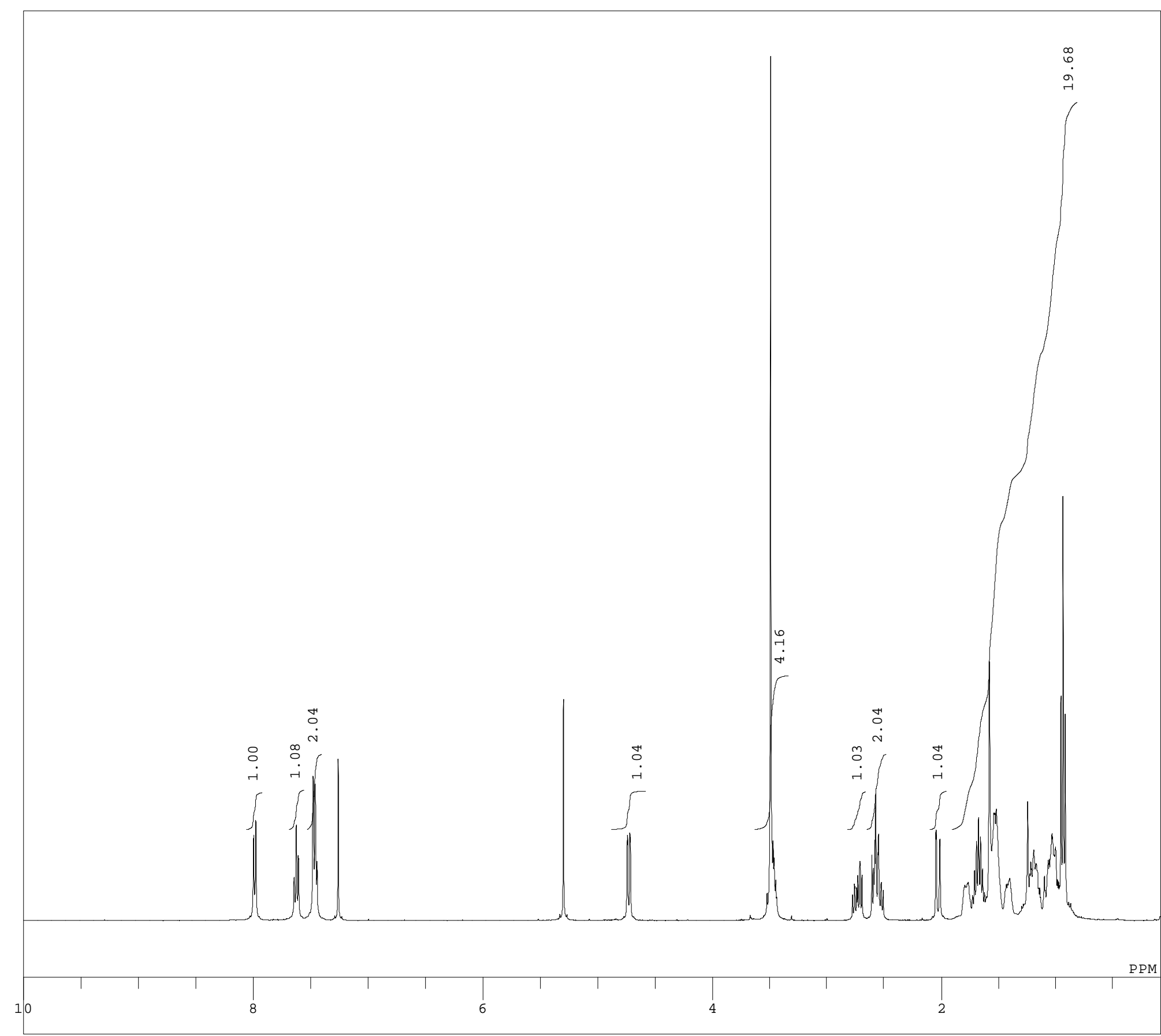

DFILE $\quad C: \backslash$ Documents and Settings $\backslash H$ Hro $\backslash f f f X f N f g f b f V$ COMNT DI

DATIM Fri Oct 27 12:47:38 2006

OBNUC $1 \mathrm{H}$

EXMOD NON

OBFRQ $\quad 399.65 \mathrm{MHZ}$

OBSET $\quad 124.00 \mathrm{KHZ}$

OBFIN $\quad 10500.0 \mathrm{HZ}$

$\begin{array}{lr}\text { POINT } & 32768 \\ \text { FREQU } & 7993.6\end{array}$

$\begin{array}{lc}\text { FREQU } & 7993.6 \mathrm{~Hz} \\ \text { SCANS } & 8\end{array}$

$\begin{array}{lr}\text { SCANS } & 8 \\ \text { ACQTM } & 4.099 \mathrm{sec}\end{array}$

PD $4.950 \mathrm{sec}$

10.0 us

IRNUC 1 H

SLVNT CDCL3

EXREF

$20.5 \mathrm{c}$

$0.00 \mathrm{ppm}$

$.20 \mathrm{~Hz}$

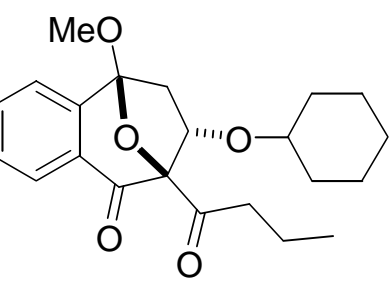




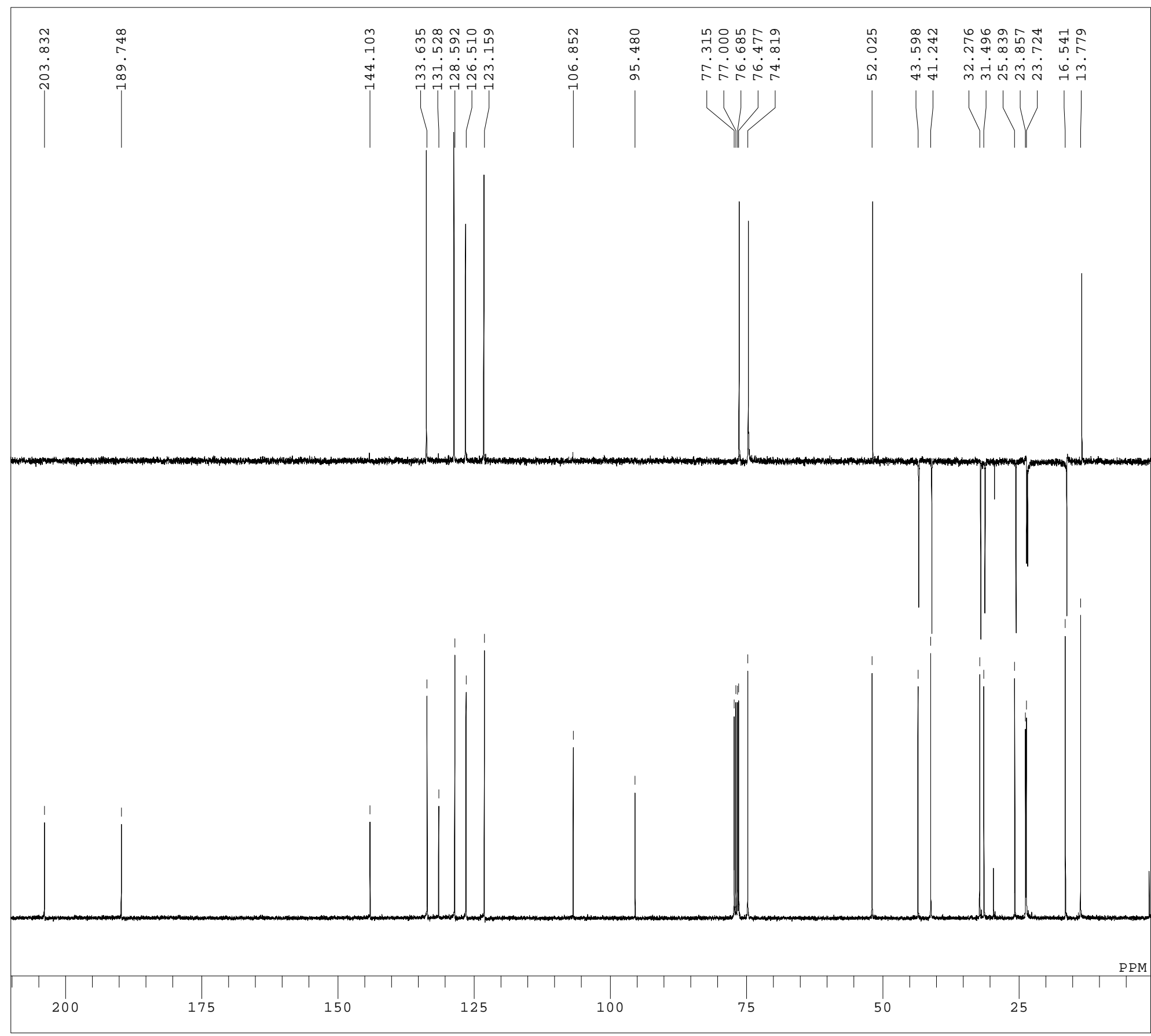

DFILE C: $\backslash$ Documents and Settings $\backslash H i r o \backslash f f f X f N f g f b f v$ COMNT DI-209-1DEPT

DATIM Sun Feb 25 01:12:57 2007

OBNUC 13C

$\begin{array}{lll}\text { EXMOD } & \text { BCM } & \\ \text { OBFRQ } & & 100.40 \mathrm{MHZ}\end{array}$

$\begin{array}{ll}\text { OBSET } & 125.00 \mathrm{KHZ}\end{array}$

OBFIN $\quad 10500.0 \mathrm{HZ}$

$\begin{array}{lr}\text { POINT } & 32768 \\ \text { FREOU } & 27322.4\end{array}$

FREQU 27322.4

SCANS 800

ACQTM $\quad 1.199 \mathrm{sec}$

$\begin{array}{lr}\text { PD } & 3.000 \mathrm{sec} \\ \text { PW1 } & 6.3 \text { us } \\ \text { IRNuc } & \end{array}$

IRNUC $\quad 1 \mathrm{H}$

CTEMP

SLVNT

6.3 us

EXREF

BF

$77.00 \mathrm{ppm}$

$77.00 \mathrm{ppm}$
$1.20 \mathrm{~Hz}$

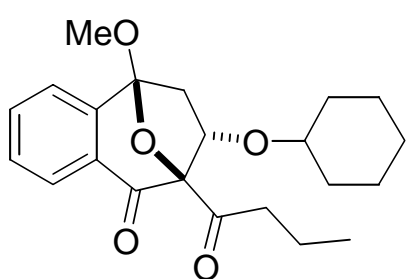




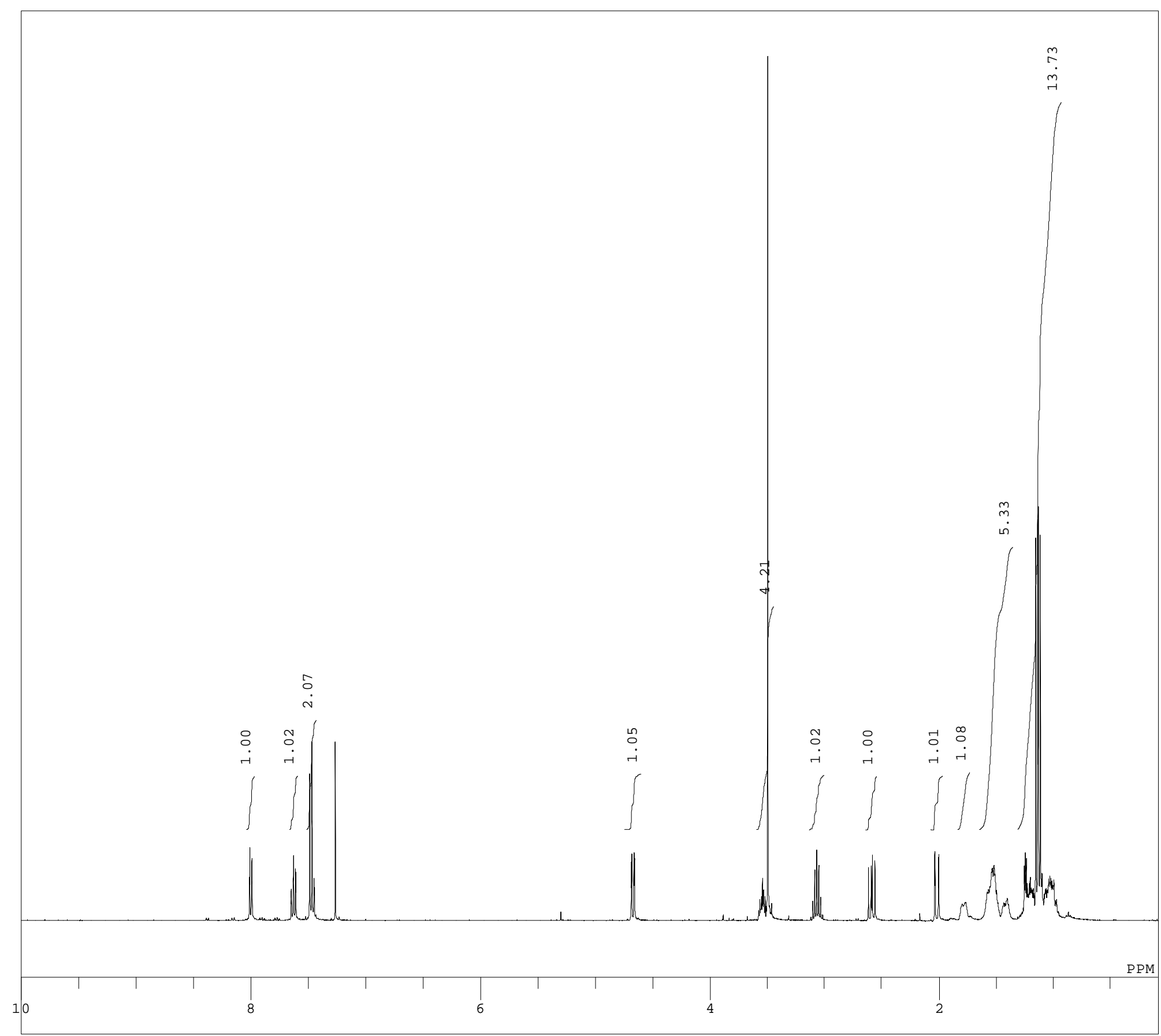

DFILE $\quad C: \backslash$ Documents and Settings $\backslash H$ Hro $\backslash f f f X f N f g f b f V$

COMNT DI

DATIM Thu Dec 14 03:19:08 200

OBNUC $1 \mathrm{H}$

EXMOD NON

OBFRQ $\quad 399.65 \mathrm{MHZ}$

OBSET $\quad 124.00 \mathrm{KHZ}$

OBFIN $\quad 10500.0 \mathrm{HZ}$

POINT 32768

$\quad 8000.0 \mathrm{~Hz}$

$\begin{array}{lr}\text { SCANS } & 8 \\ \text { ACQTM } & 4.096 \mathrm{sec}\end{array}$

$\begin{array}{ll}\text { ACQTM } & 4.096 \mathrm{sec} \\ \text { PD } & 4.950 \mathrm{sec}\end{array}$

PW1 10.

IRNUC $\quad 1 \mathrm{H}$

SLVNT CDCL3

SLVNT CDCL

EXREF

$0.00 \mathrm{ppm}$ .24
18

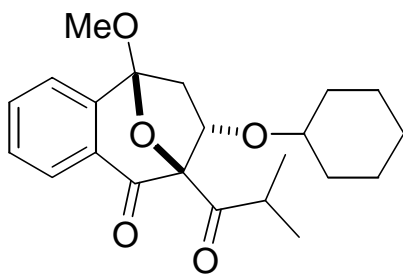




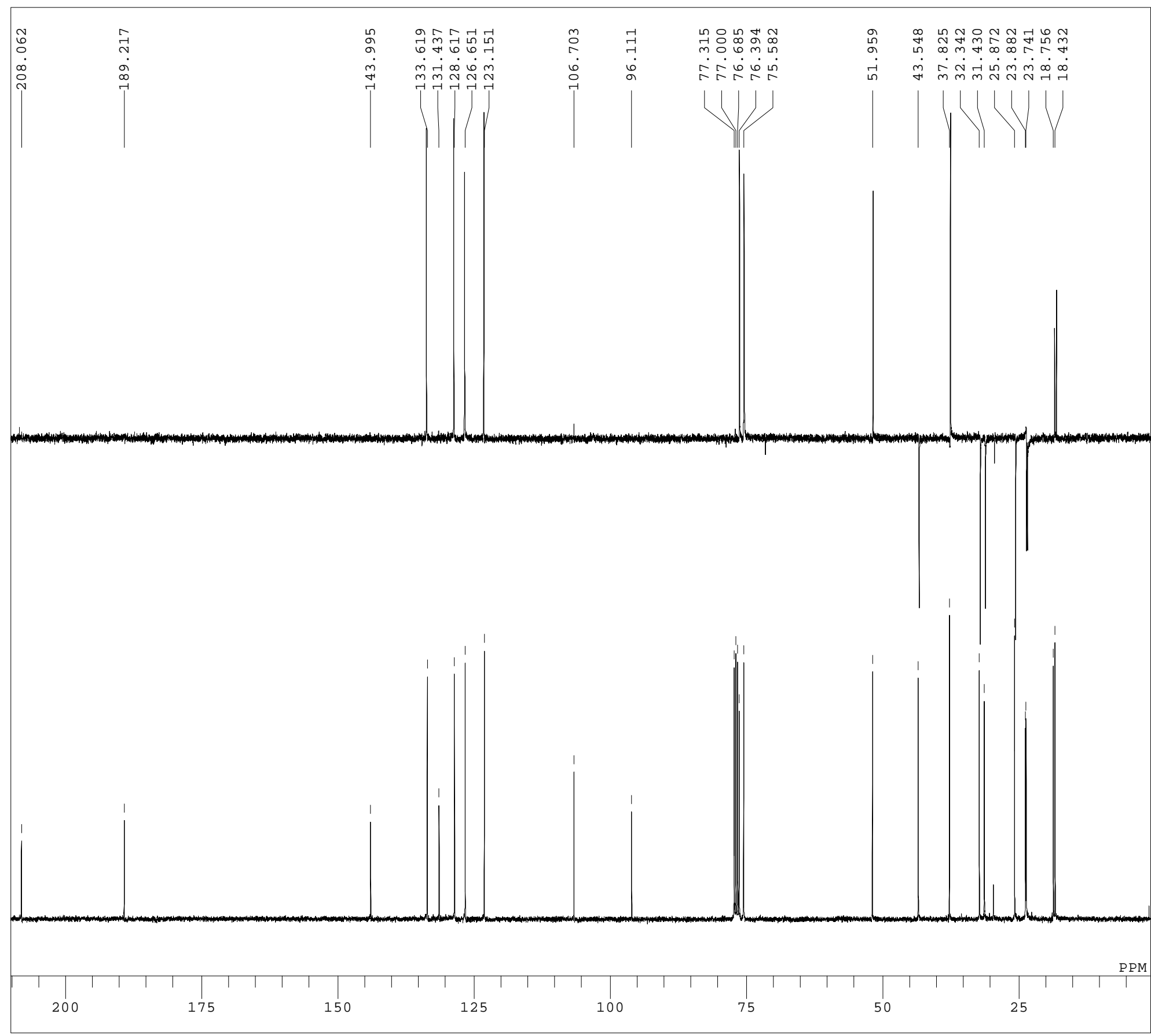

DFILE C: $\backslash$ Documents and Settings $\backslash H i r o \backslash f f f X f N f g f b f v \backslash$ COMNT DI-216-1DEPT

DATIM Sat Feb 24 20:57:41 200

OBNUC $13 \mathrm{C}$

$\begin{array}{lll}\text { EXMOD } & \text { BCM } & \\ \text { OBFRQ } & & 100.40 \mathrm{MHZ}\end{array}$

OBSET $\quad 125.00 \mathrm{KHZ}$

OBFIN $\quad 10500.0 \mathrm{~Hz}$

$\begin{array}{lr}\text { POINT } & 32768 \\ \text { FREQU } & 27322.4\end{array}$

$\begin{array}{lr}\text { FREQU } & 27322.4 \\ \text { SCANS } & 800\end{array}$

$\begin{array}{lr}\text { SCANS } & 800 \\ \text { ACQTM } & 1.199 \mathrm{sec}\end{array}$

$\begin{array}{ll}\text { ACQTM } & 1.199 \mathrm{sec} \\ \mathrm{PD} & 3.000 \mathrm{sec}\end{array}$

PW1 6.3 us

IRNUC

SLVNT CDCL3

SLVNT

$26.0 \mathrm{C}$

EXRE

BF

$77.00 \mathrm{ppm}$
$1.20 \mathrm{~Hz}$
$31 \mathrm{~m}$

$.20 \mathrm{~Hz}$
31

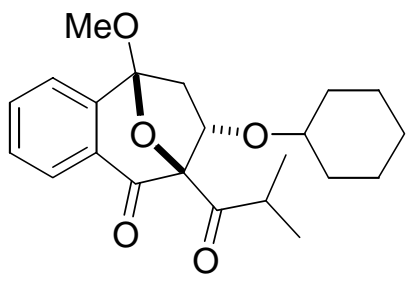




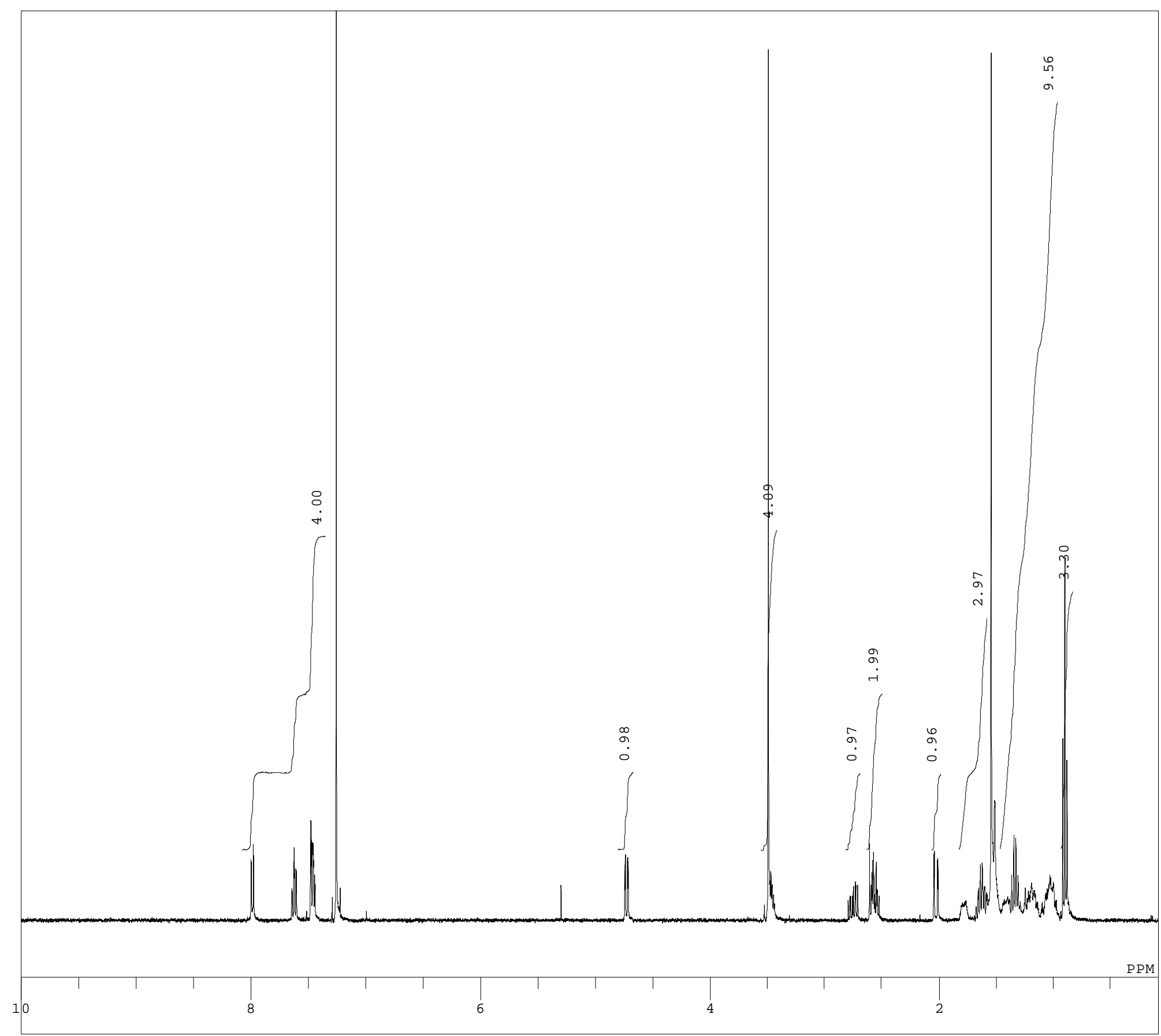

DFILE $\quad C: \backslash$ Documents and Settings $\backslash H i r o \backslash f f f X f N f g f b f v \backslash$ COMNT SH

DATIM Wed Apr 25 13:16:40 2007

OBNUC $1 \mathrm{H}$

EXMOD NON

OBFRQ $\quad 399.65 \mathrm{MHZ}$

OBSET $\quad 124.00 \mathrm{KHZ}$

OBFIN $\quad 10500.0 \mathrm{HZ}$

$\begin{array}{lr}\text { POINT } & 32768 \\ \text { FREQU } & 7993.6\end{array}$

$\begin{array}{lc}\text { FREQU } & 7993.6 \mathrm{~Hz} \\ \text { SCANS } & 8\end{array}$

$\begin{array}{lr}\text { SCANS } & 8 \\ \text { ACQTM } & 4.099 \mathrm{sec}\end{array}$

PD $4.950 \mathrm{sec}$

PW1 10.0 us

IRNUC $\quad 1 \mathrm{H}$

SLVNT CDCL3

EXREF

$21.1 \mathrm{c}$

$0.00 \mathrm{ppm}$ $.24 \mathrm{~Hz}$

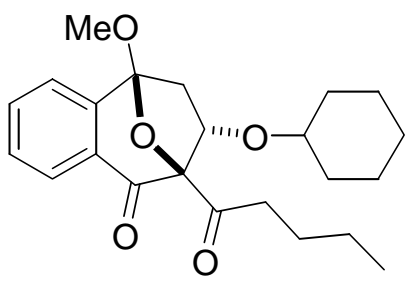




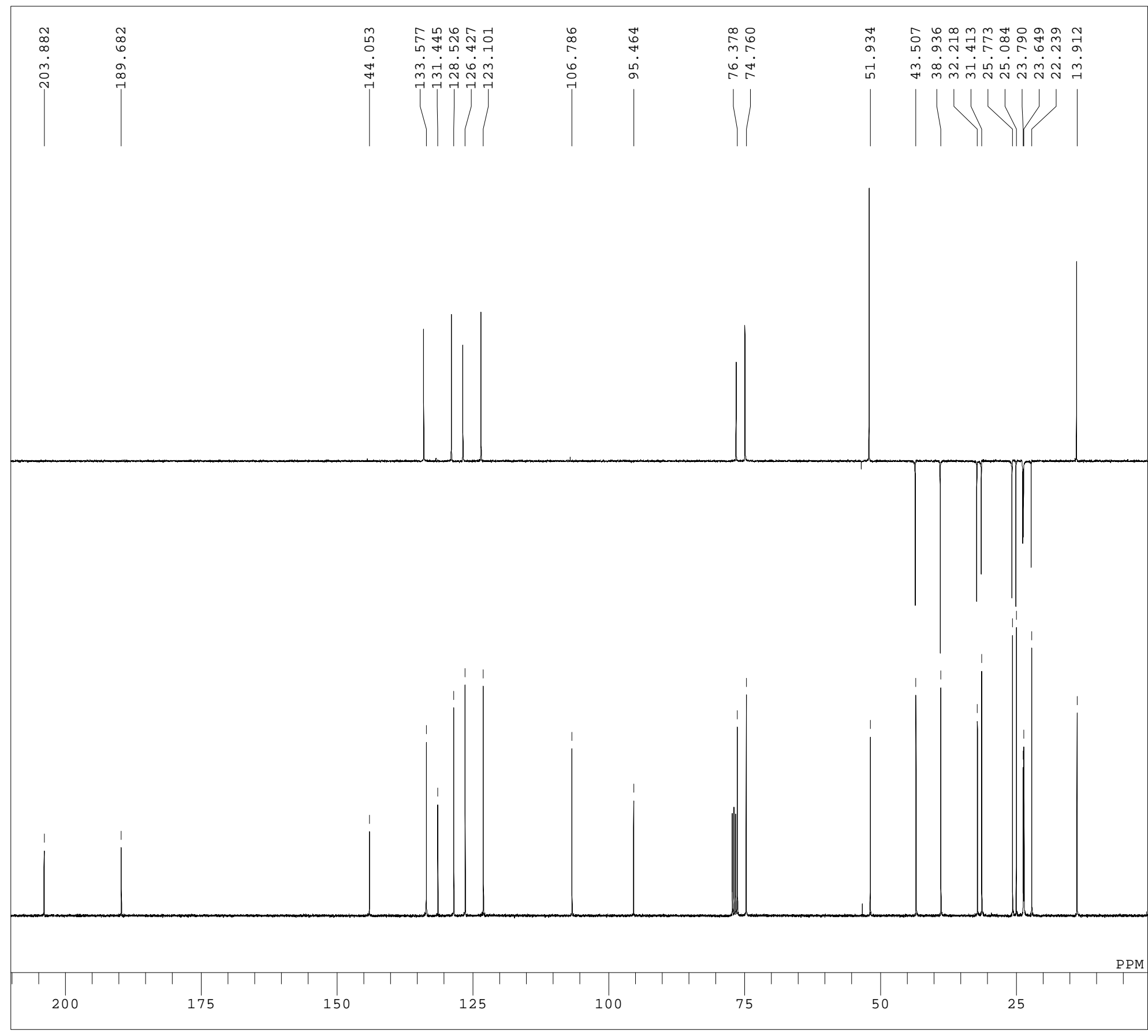

DFILE C: $\backslash$ Documents and Settings $\backslash H i r o \backslash f f f X f N f g f b f v \backslash$ COMNT SH

DATIM Wed Apr 25 18:19:50 2007

OBNUC 13C

EXMOD BCM

OBFRQ $\quad 100.40 \mathrm{MHz}$

OBSET $\quad 125.00 \mathrm{KHZ}$

OBFIN $\quad 10500.0 \mathrm{~Hz}$

POINT 32768

FREQU 27322.4

SCANS 800

ACQTM $\quad 1.199 \mathrm{sec}$

$\begin{array}{lr}\text { PD } & 3.000 \mathrm{sec} \\ \text { PW1 } & 6.3 \text { us }\end{array}$

IRNUC $1 \mathrm{H}$

SLEMPT CDCL3

STRE

6.3 us

EXREF

BAIN

$77.00 \mathrm{ppm}$ 83
30

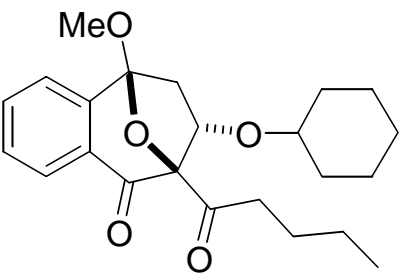




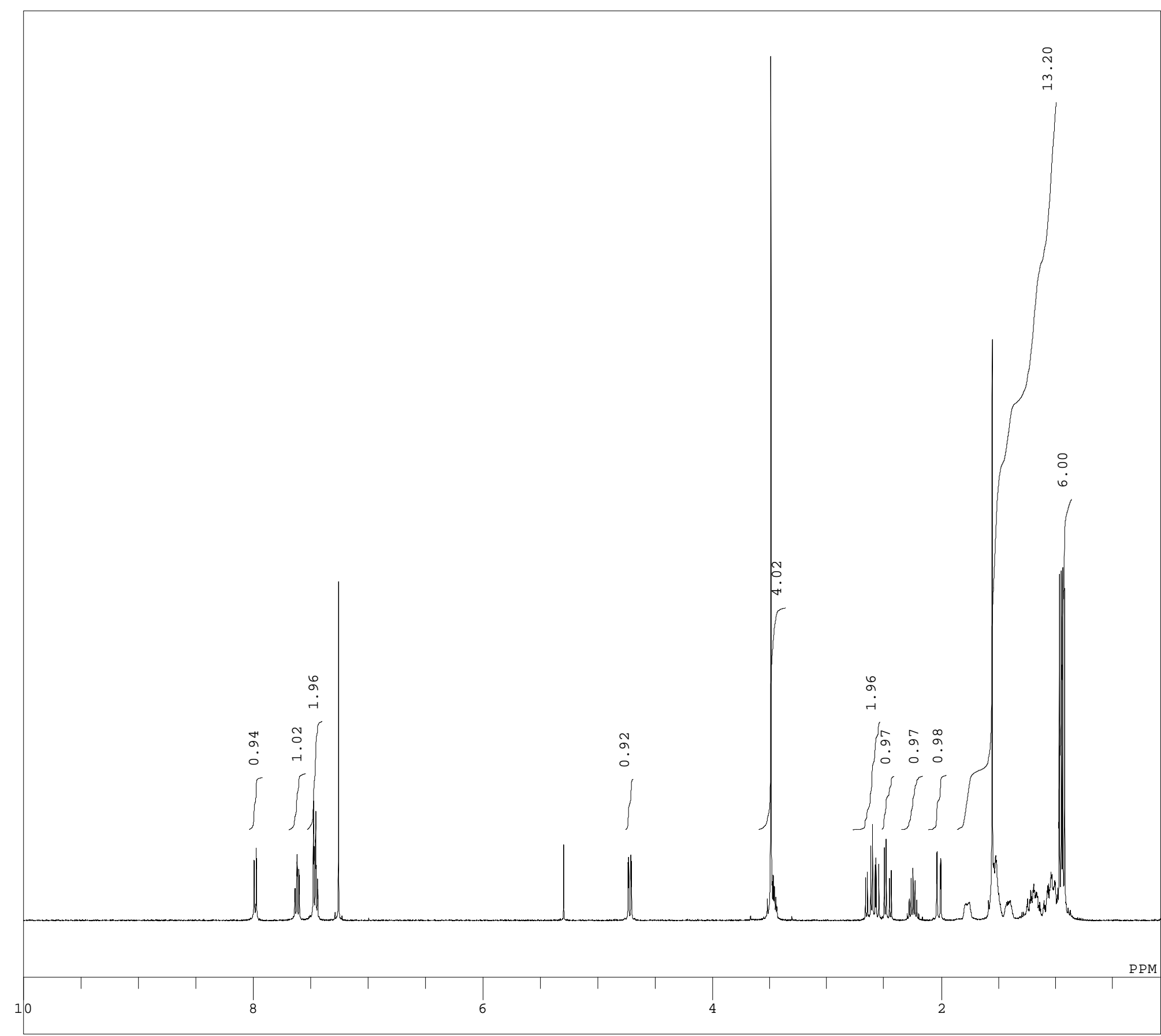

DFILE $\quad C: \backslash$ Documents and Settings $\backslash H i r o \backslash f f f X f N f g f b f v \backslash$ COMNT SH

DATIM Tue Apr 03 10:06:55 2007

OBNUC $1 \mathrm{H}$

EXMOD NON

OBFRQ $\quad 399.65 \mathrm{MHZ}$

OBSET $\quad 124.00 \mathrm{KHZ}$

OBFIN $\quad 10500.0 \mathrm{HZ}$

$\begin{array}{lr}\text { POINT } & 32768 \\ \text { FREQU } & 7993.6\end{array}$

$\begin{array}{lc}\text { FREQU } & 7993.6 \mathrm{~Hz} \\ \text { SCANS } & 8\end{array}$

$\begin{array}{lr}\text { SCANS } & 8 \\ \text { ACQTM } & 4.099 \mathrm{sec}\end{array}$

PD $\quad 4.950 \mathrm{sec}$

PW1 10.0 us

IRNUC $\quad 1 \mathrm{H}$

SLVNT CDCL3

EXREF

$24.0 \mathrm{C}$

$0.00 \mathrm{ppm}$ $.24 \mathrm{~Hz}$

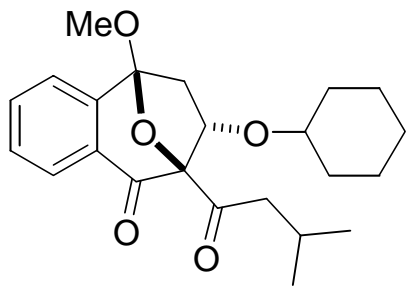




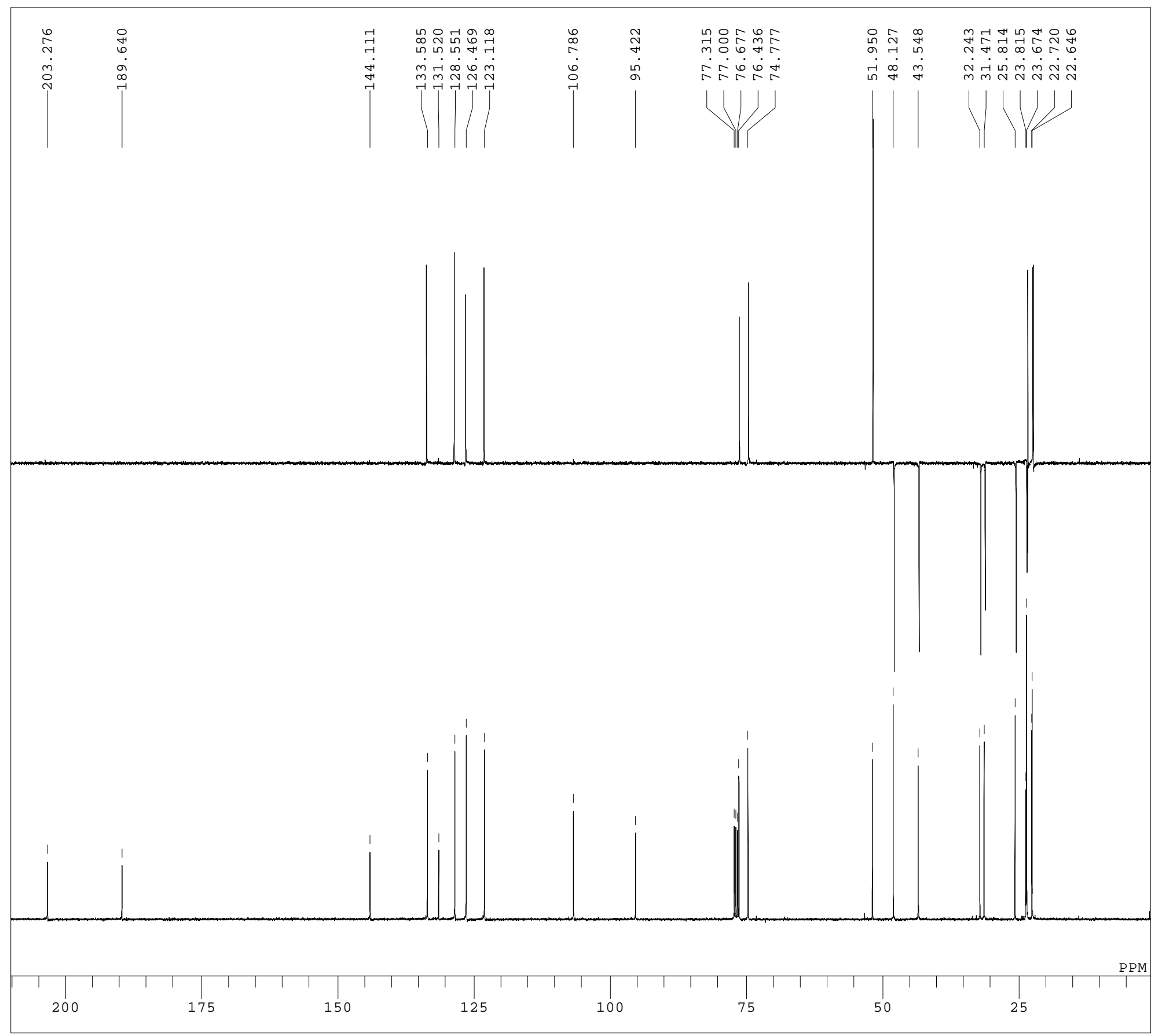

DFILE C: $\backslash$ Documents and Settings $\backslash H i r o \backslash f f f X f N f g f b f v \backslash$ COMNT SH

DATIM Tue Apr 03 20:49:08 2007

OBNUC 13C

EXMOD BCM

$\begin{array}{ll}\text { OBFRQ } & 100.40 \mathrm{MHz}\end{array}$

$\begin{array}{ll}\text { OBSET } & 125.00 \mathrm{KHZ}\end{array}$

OBFIN $\quad 10500.0 \mathrm{~Hz}$

POINT 32768

FREQU $27322.4 \mathrm{~Hz}$

SCANS $\quad 640$

ACQTM $\quad 1.199 \mathrm{sec}$

$\begin{array}{lr}\text { PD } & 3.000 \mathrm{sec} \\ \text { PW1 } & 6.3 \text { us }\end{array}$

IRNUC $1 \mathrm{H}$

CTEMP 26.6

SLVNT CDCL3

EXREF $\quad 77.00 \mathrm{ppm}$

$1.20 \mathrm{~Hz}$

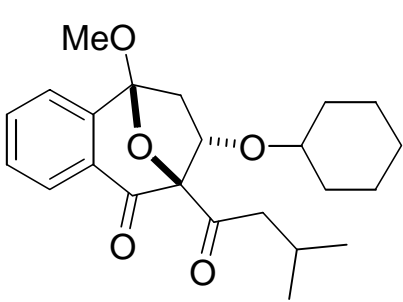




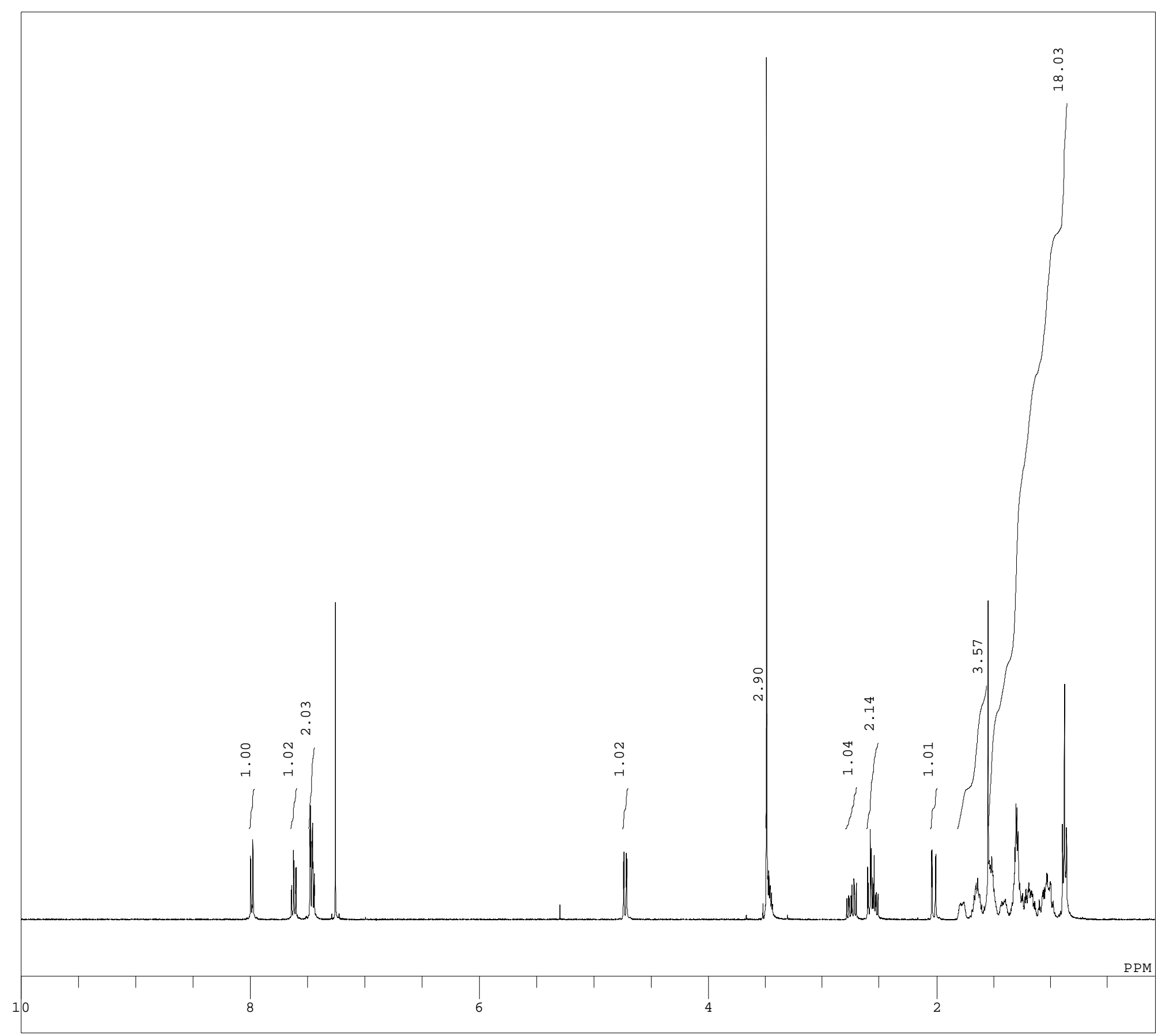

DFILE $\quad C: \backslash$ Documents and Settings $\backslash$ Hiro $\backslash f f f X f N f g f b f v \backslash$ COMNT MO

DATIM Mon Mar 19 20:07:56 2007

OBNUC $1 \mathrm{H}$

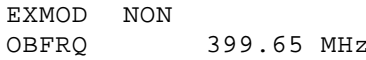

$\begin{array}{ll}\text { OBFRQ } & 399.65 \mathrm{MHz} \\ \text { OBSET } & 124.00 \mathrm{KHz}\end{array}$

$\begin{array}{lr}\text { OBSET } & 124.00 \mathrm{KHz} \\ \text { OBFIN } & 10500.0 \mathrm{~Hz}\end{array}$

POINT $\quad 32768$

FREQU $\quad 7993.6 \mathrm{~Hz}$

$\begin{array}{lr}\text { SCANS } & 8 \\ \text { ACOTM } & 4.099 \mathrm{sec}\end{array}$

$\begin{array}{ll}\text { ACQTM } & 4.099 \mathrm{sec} \\ \text { PD } & 4.950 \mathrm{sec}\end{array}$

PW1 10.0 us

$\begin{array}{ll}\text { IRNUC } & 1 \mathrm{H} \\ \text { CTEMP } & \end{array}$

SLVNT CDCL3

EXREF

$23.4 \mathrm{C}$

$0.00 \mathrm{ppm}$

$.12 \mathrm{~Hz}$

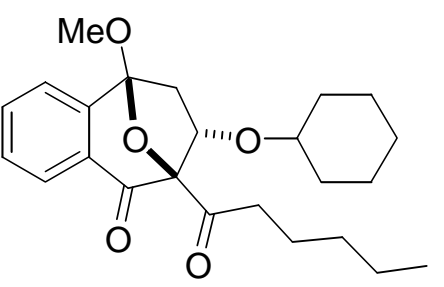




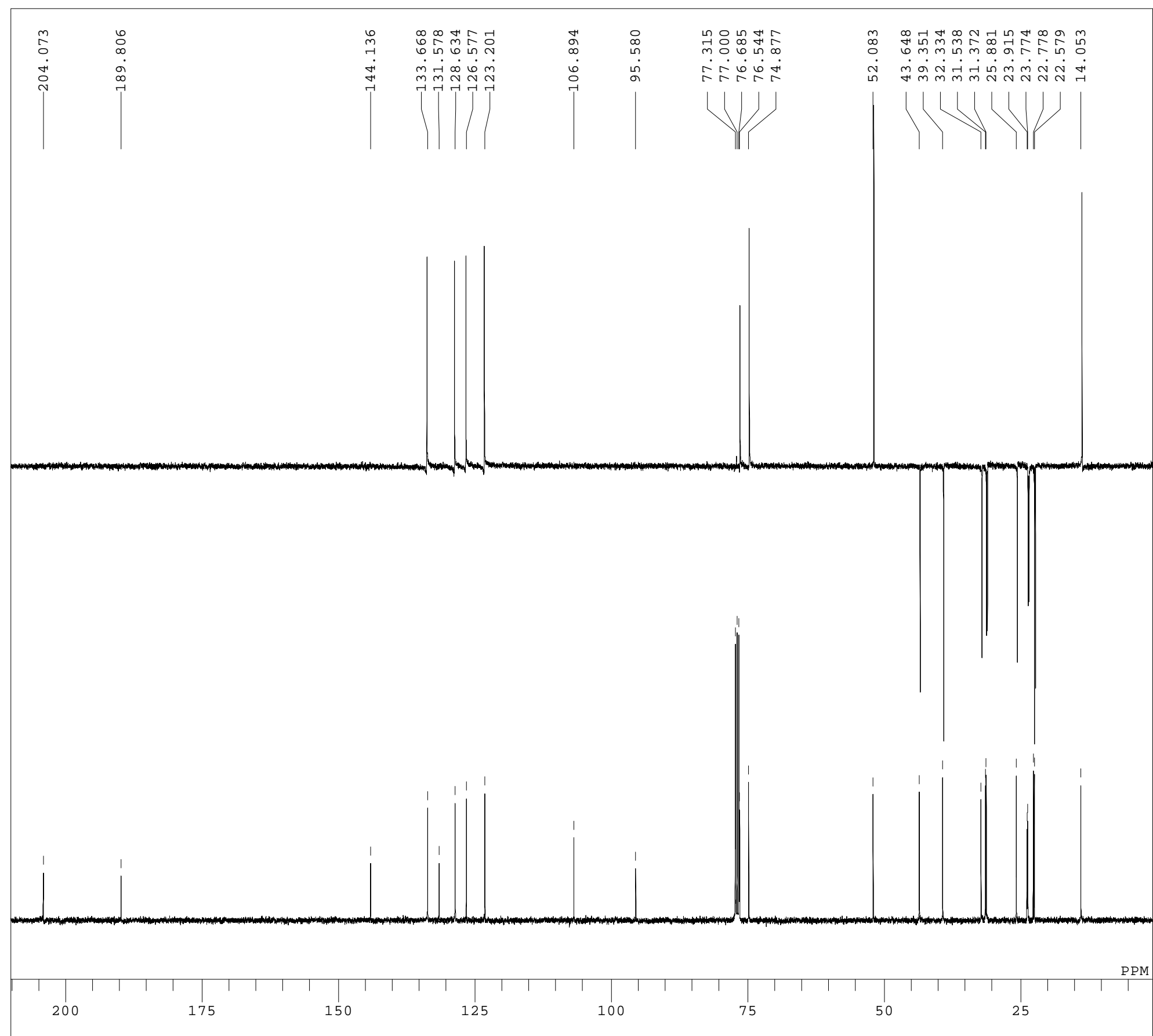

DFILE C: $\backslash$ Documents and Settings $\backslash$ Hiro $\backslash f f f X f N f g f b f v \backslash$ COMNT MO

DATIM Wed Apr 04 22:00:57 2007

OBNUC $13 \mathrm{C}$

XMOD BCM

OBFRQ $\quad 100.40 \mathrm{MHz}$

OBSET $\quad 125.00 \mathrm{KHZ}$

OBFIN $\quad 10500.0 \mathrm{~Hz}$

$\begin{array}{lr}\text { POINT } & 32768 \\ \text { FREQU } & 27322.4\end{array}$

$\begin{array}{lr}\text { SCANS } & 1000\end{array}$

ACQTM $\quad 1.199 \mathrm{sec}$

$\begin{array}{lll}\mathrm{PD} & 3.000 \mathrm{sec}\end{array}$

PW1 6.3 us

IRNUC

26.5 c

SLVNT CDCL3

EXRE

$77.00 \mathrm{ppm}$

31

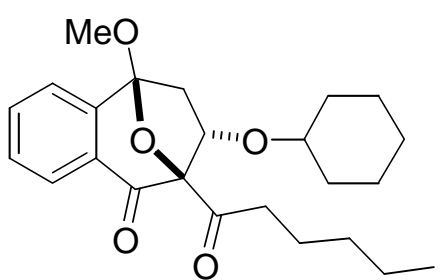




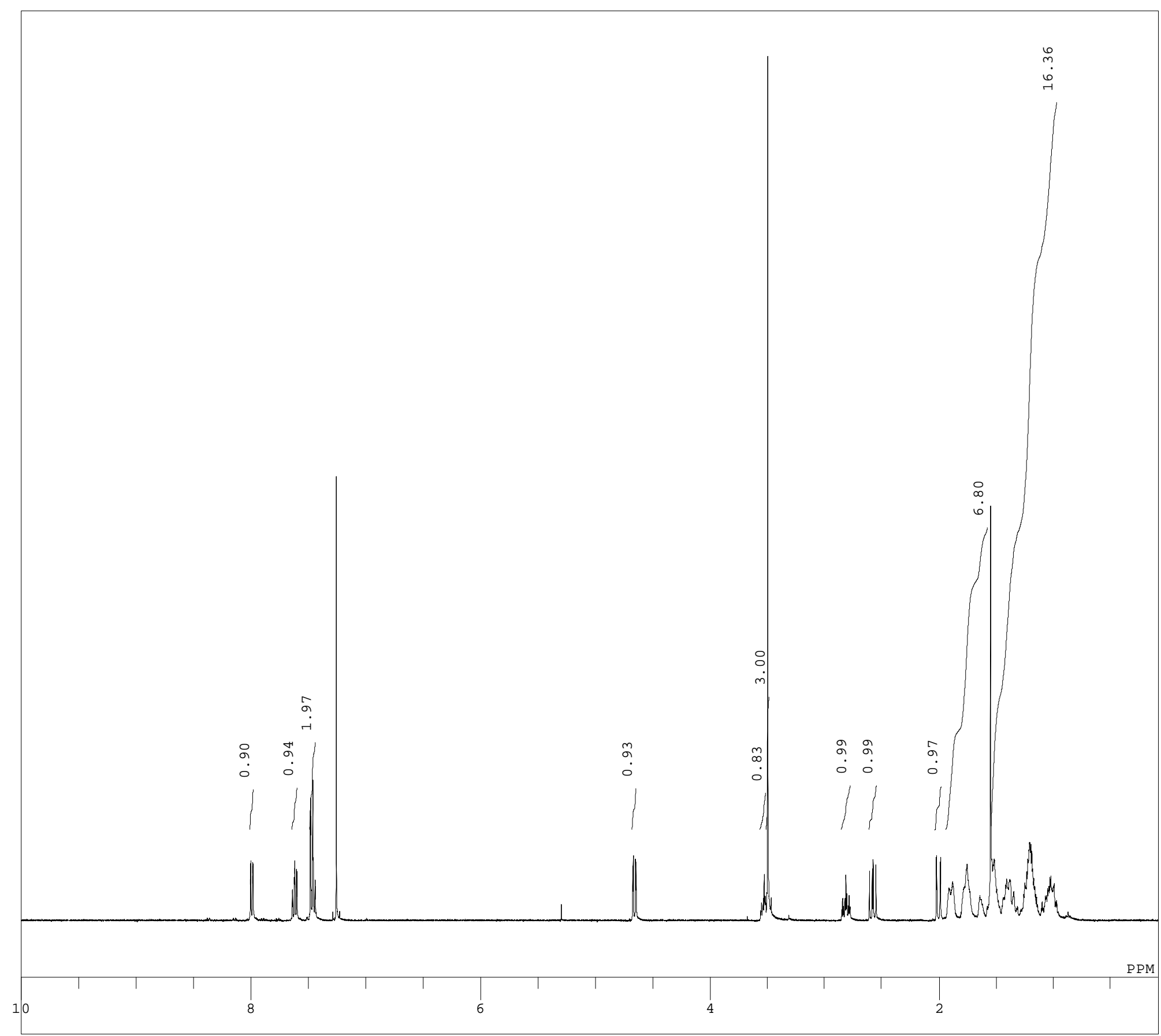

DFILE $\quad C: \backslash$ Documents and Settings $\backslash$ Hiro $\backslash f f f X f N f g f b f v \backslash$ COMNT MO

DATIM Mon Apr 02 17:31:40 2007

OBNUC $1 \mathrm{H}$

EXMOD NON

OBFRQ $\quad 399.65 \mathrm{MHZ}$

OBSET $\quad 124.00 \mathrm{KHZ}$

OBFIN $\quad 10500.0 \mathrm{HZ}$

$\begin{array}{lr}\text { POINT } & 32768 \\ \text { FREQU } & 7993.6\end{array}$

$\begin{array}{lc}\text { FREQU } & 7993.6 \mathrm{~Hz} \\ \text { SCANS } & 8\end{array}$

$\begin{array}{lr}\text { SCANS } & 8 \\ \text { ACQTM } & 4.099 \mathrm{sec}\end{array}$

PD $4.950 \mathrm{sec}$

10.0 us

$\begin{array}{lll}\text { IRNUC } & 1 \mathrm{H} & 24.1 \mathrm{C}\end{array}$

SLVNT CDCL3

$\begin{array}{lll}\text { SLVNT CDCL3 } & \\ \text { EXREF } & & 0.00 \mathrm{ppm}\end{array}$ $0.00 \mathrm{ppm}$
$0.12 \mathrm{~Hz}$

$\mathrm{MeO}$

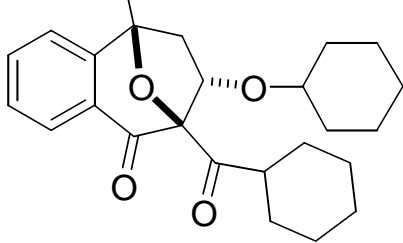




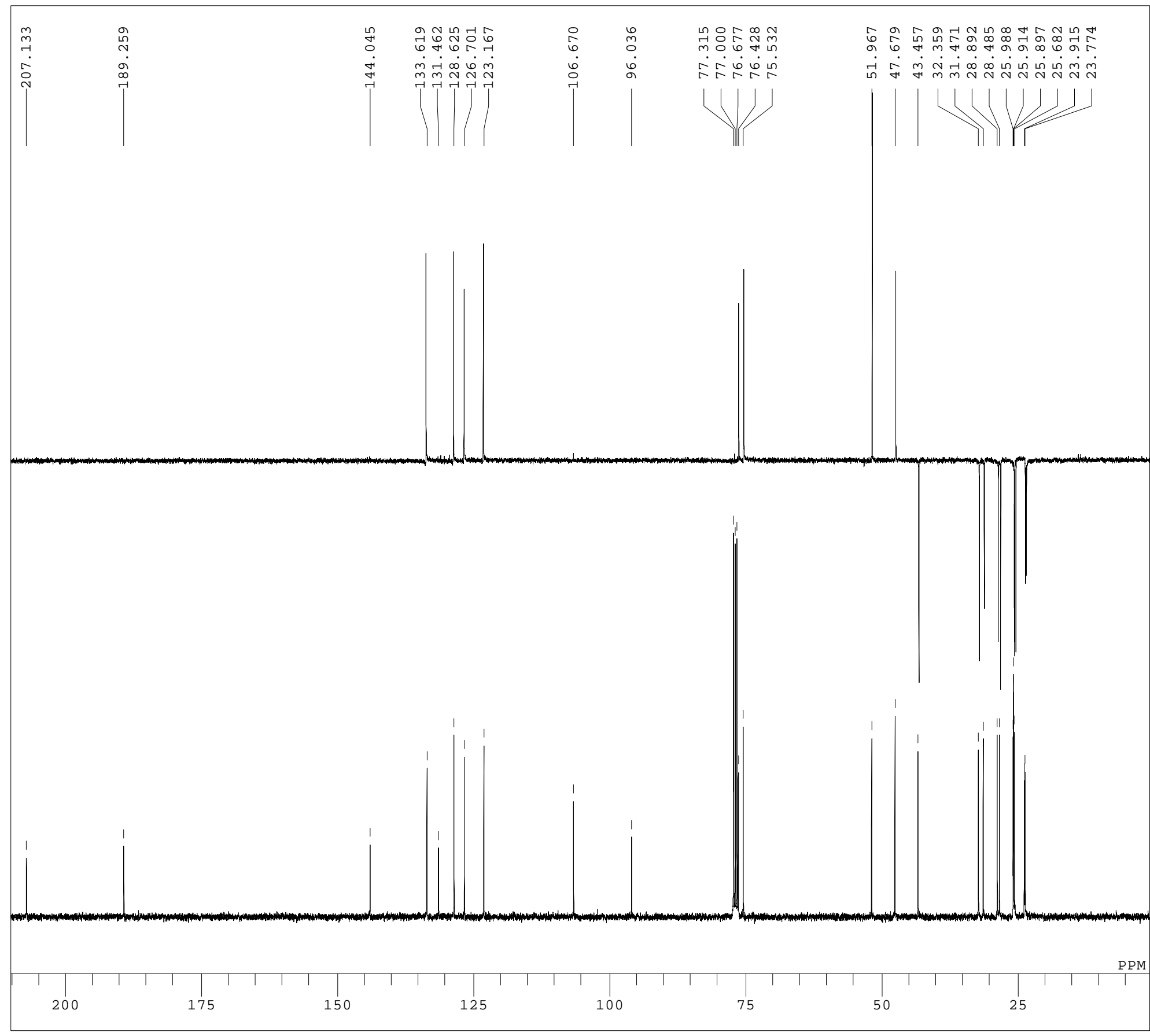

DFILE C: $\backslash$ Documents and Settings $\backslash H i r o \backslash f f f X f N f g f b f v$ COMNT MO

DATIM Thu Apr 05 22:27:07 2007

OBNUC $13 \mathrm{C}$

EXMOD BCM

OBFRQ $\quad 100.40 \mathrm{MHz}$

OBSET $\quad 125.00 \mathrm{KHZ}$

OBFIN $\quad 10500.0 \mathrm{~Hz}$

$\begin{array}{rr}\text { POINT } & 32768 \\ \text { FREQU } & 27322.4\end{array}$

$\begin{array}{rr}\text { FREQU } & 27322.4 \\ \text { SCANS } & 1000\end{array}$

$\begin{array}{lr}\text { SCANS } & 1000 \\ \text { ACQTM } & 1.199 \mathrm{sec}\end{array}$

$\begin{array}{ll}A C Q T M & 1.199 \mathrm{sec} \\ \mathrm{PD} & 3.000 \mathrm{sec}\end{array}$

PW1 6.3 us

IRNUC

$26.3 \mathrm{C}$

SLVNT CDCL3

EXREF

$77.00 \mathrm{ppm}$

$\mathrm{MeO}$

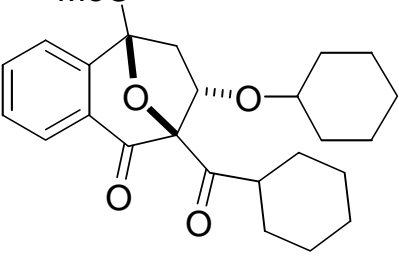




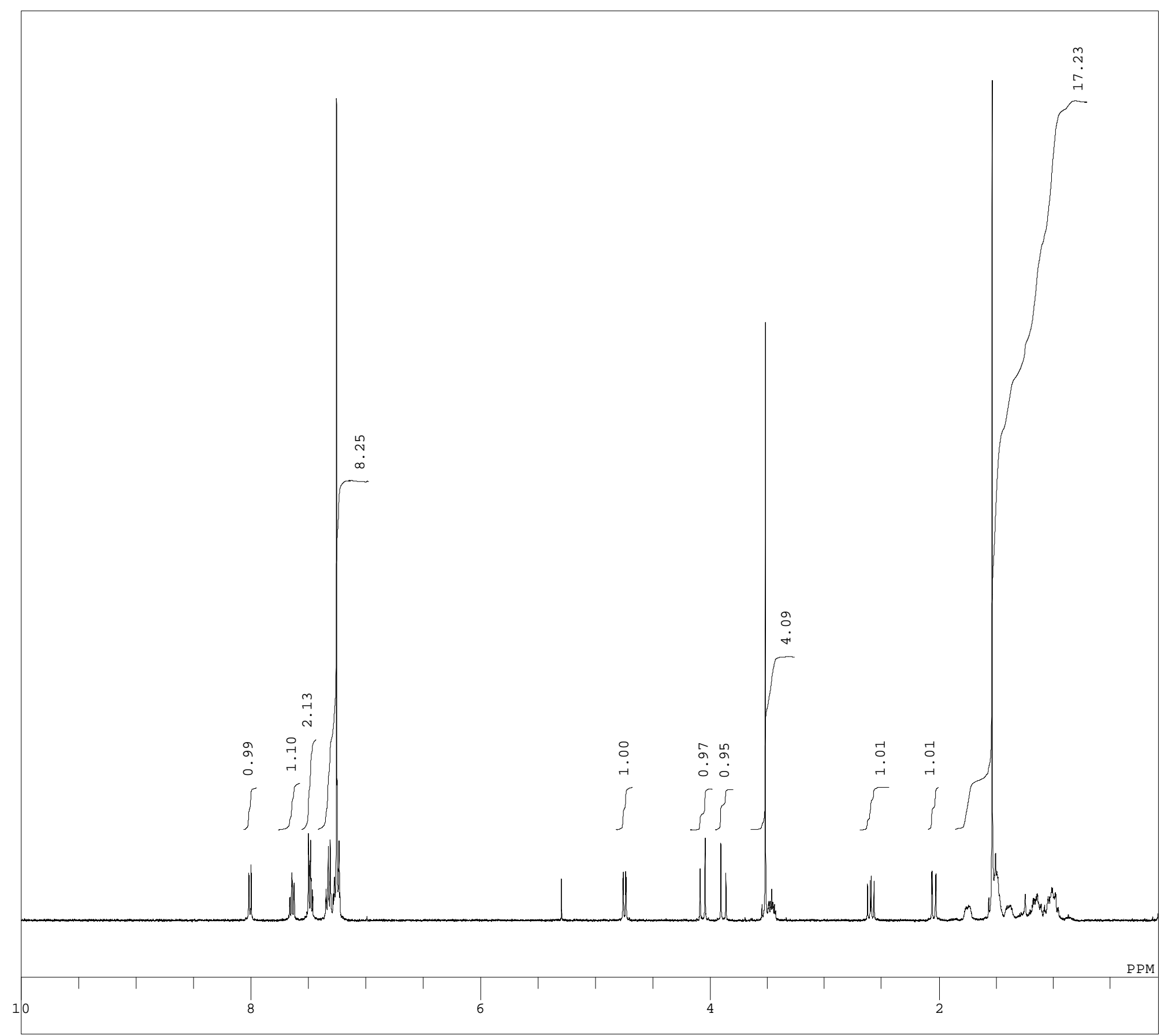

DFILE $\quad C: \backslash$ Documents and Settings $\backslash H i r o \backslash f f f X f N f g f b f v \backslash$ COMNT SH

DATIM Fri Apr 06 09:59:06 2007

OBNUC $1 \mathrm{H}$

EXMOD NON

OBFRQ $\quad 399.65 \mathrm{MHZ}$

OBSET $\quad 124.00 \mathrm{KHZ}$

OBFIN $\quad 10500.0 \mathrm{~Hz}$

POINT 32768

FREQU $7993.6 \mathrm{~Hz}$

$\begin{array}{lr}\text { SCANS } & 8 \\ \text { ACOTM } & 4.099 \mathrm{sec}\end{array}$

$\begin{array}{ll}\text { ACQTM } & 4.099 \mathrm{sec} \\ \text { PD } & 4.950 \mathrm{sec}\end{array}$

PW1 10.0 us

IRNUC $\quad 1 \mathrm{H}$

SLVNT CDCL3

SLVNT CDCL3

EXREF

$0.00 \mathrm{ppm}$ $.24 \mathrm{~Hz}$
24

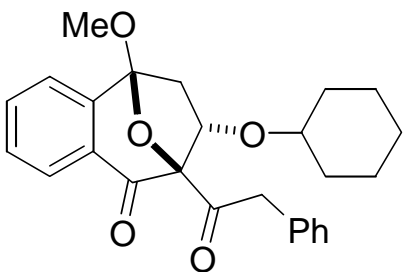




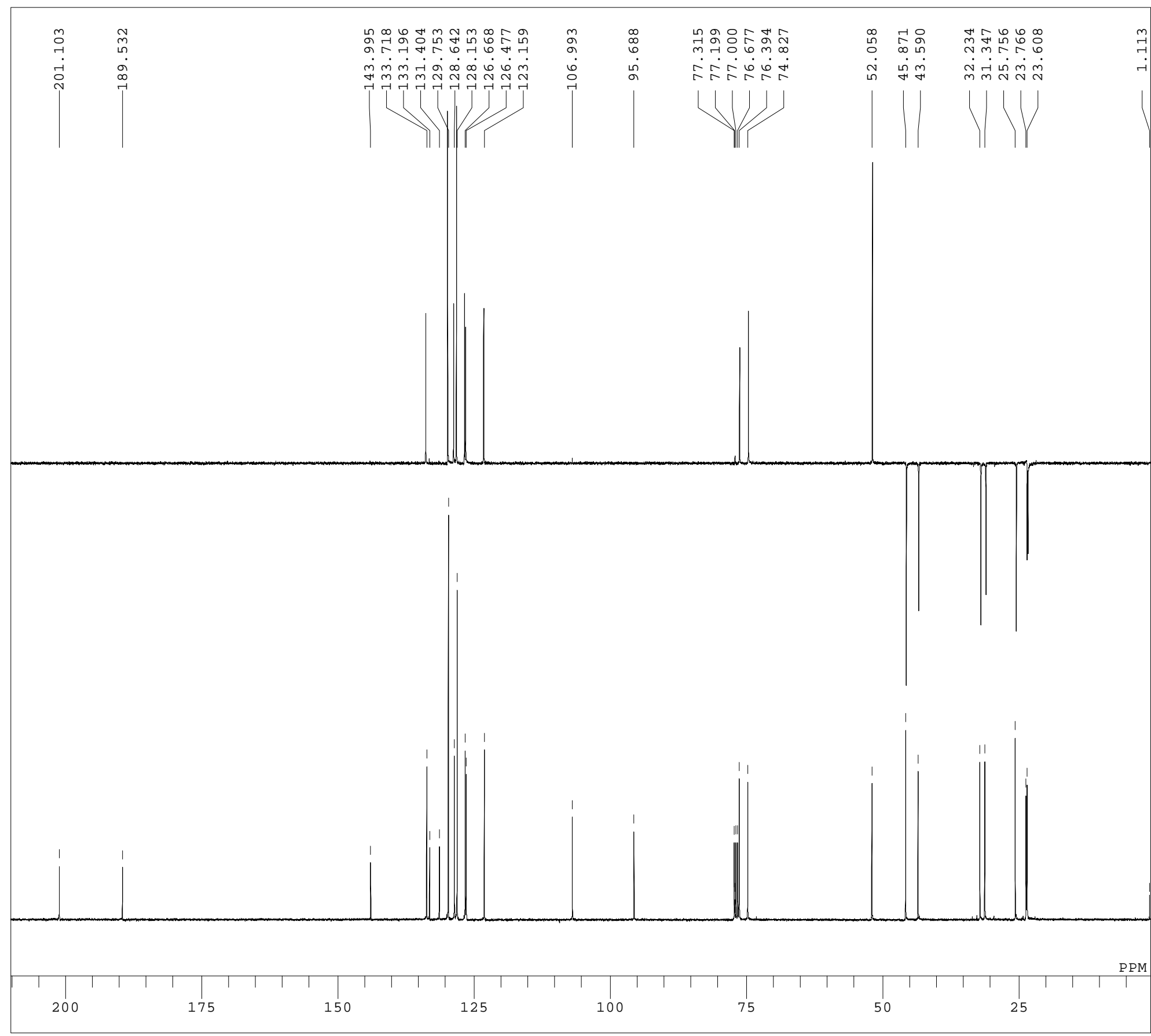

DFILE $\quad C: \backslash$ Documents and Settings $\backslash H i r o \backslash f f f X f N f g f b f v \backslash$

COMNT SH

DATIM Wed Apr 18 16:00:20 2007

OBNUC $13 \mathrm{C}$

EXMOD BCM

$\begin{array}{ll}\text { OBFRQ } & 100.40 \mathrm{MHz}\end{array}$

$\begin{array}{ll}\text { OBSET } & 125.00 \mathrm{KHZ}\end{array}$

OBFIN $\quad 10500.0 \mathrm{HZ}$

POINT 32768

FREQU 27322.4

SCANS 800

ACQTM $\quad 1.199 \mathrm{sec}$

$\begin{array}{lr}\text { PD } & 3.000 \mathrm{sec} \\ \text { PW1 } & 6.3 \text { us }\end{array}$

IRNUC $\quad 1 \mathrm{H}$

STEMPT CDCL3

STRE

$\mathrm{BF}$

6.3 us

$77.00 \mathrm{ppm}$

20
31

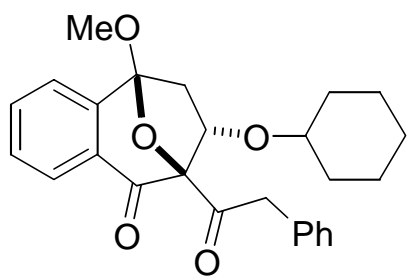




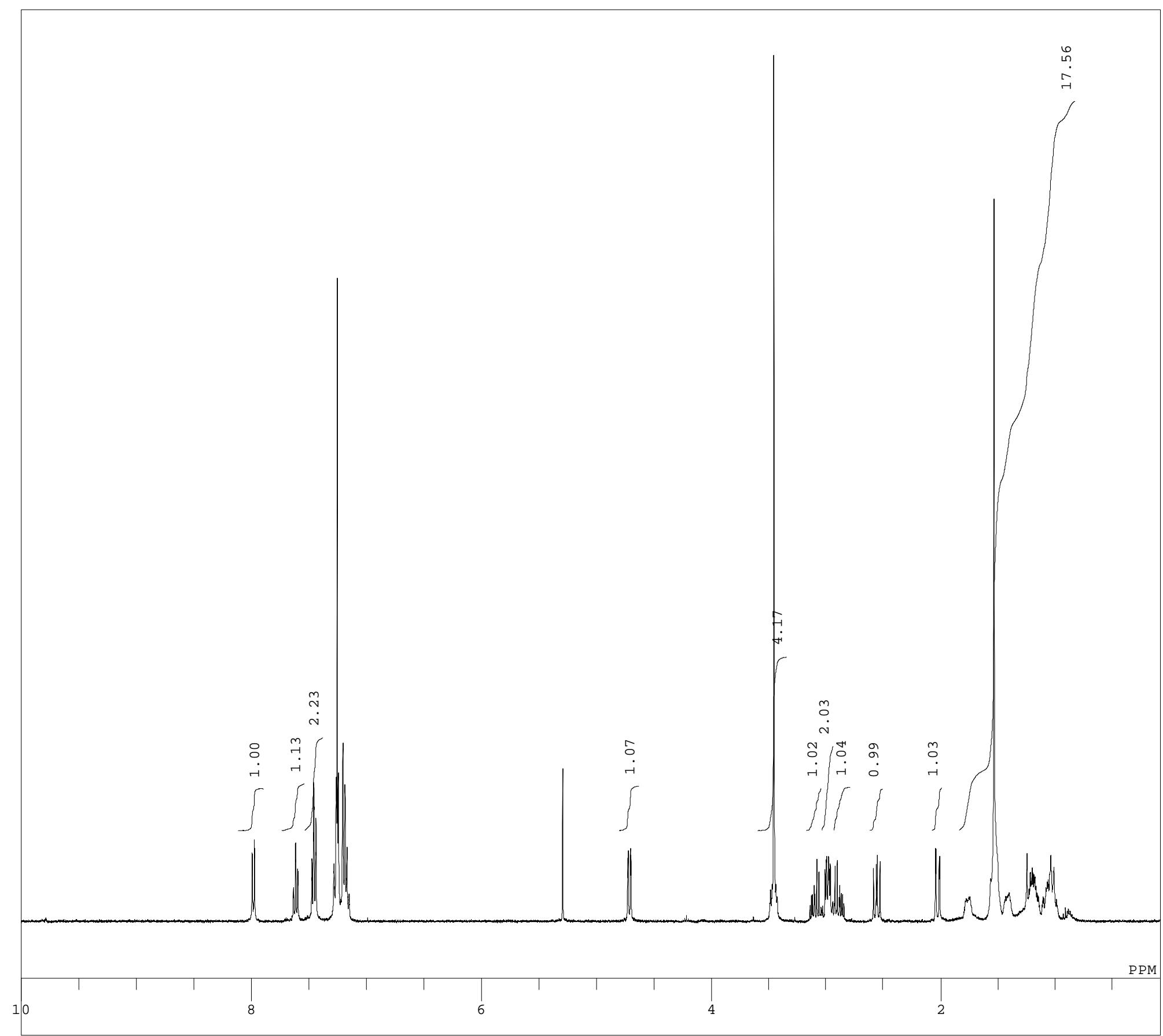

DFILE $\quad C: \backslash$ Documents and Settings $\backslash$ Hiro $\backslash f f f X f N f g f b f v \backslash$ COMNT SH

DATIM Wed Mar 28 14:03:18 2007

OBNUC $1 \mathrm{H}$

EXMOD NON

OBFRQ $\quad 399.65 \mathrm{MHZ}$

OBSET $\quad 124.00 \mathrm{KHZ}$

OBFIN $\quad 10500.0 \mathrm{HZ}$

POINT 32768

FREQU $\quad 7993.6 \mathrm{~Hz}$

$\begin{array}{lr}\text { SCANS } & 8 \\ \text { ACQTM } & 4.099 \mathrm{sec}\end{array}$

$\begin{array}{ll}\text { ACQTM } & 4.099 \mathrm{sec} \\ \text { PD } & 4.950 \mathrm{sec}\end{array}$

PW1 10.0 us

IRNUC $\quad 1 \mathrm{H}$

SLVNT CDCL3

EXREF

$26.1 \mathrm{c}$

$0.00 \mathrm{ppm}$ 24
23

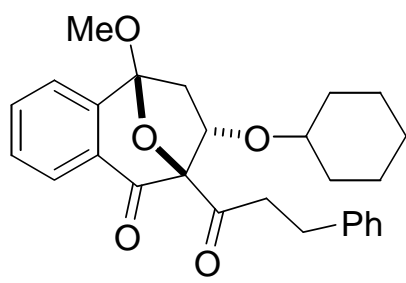




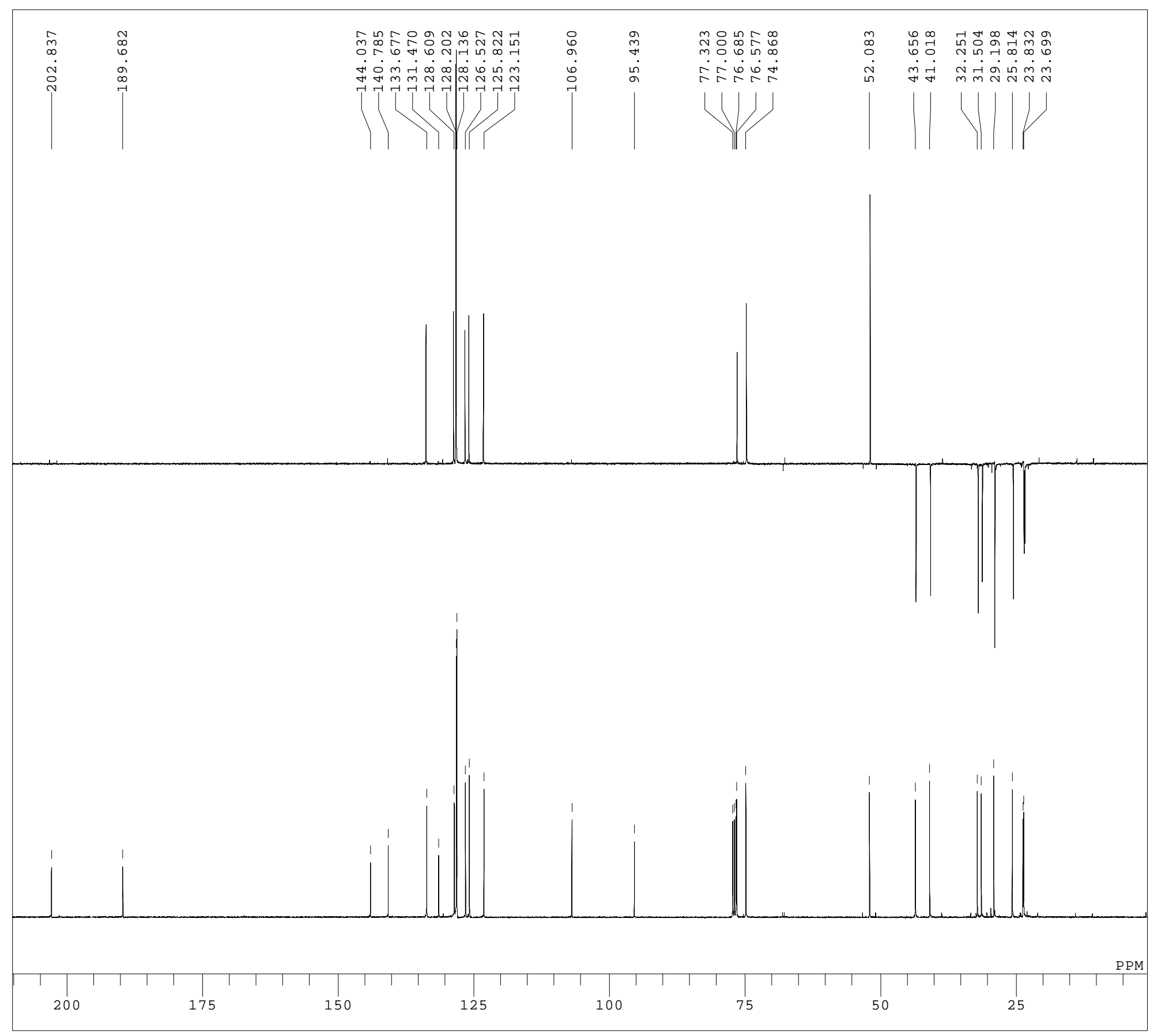

DFILE $\quad C: \backslash$ Documents and Settings $\backslash$ Hiro $\backslash f f f X f N f g f b f v \backslash$

COMNT SH

DATIM Wed Apr 04 01:56:57 2007

OBNUC $13 \mathrm{C}$

EXMOD BCM

$\begin{array}{ll}\text { OBFRQ } & 100.40 \mathrm{MHz}\end{array}$

$\begin{array}{ll}\text { OBSET } & 125.00 \mathrm{KHZ}\end{array}$

OBFIN $\quad 10500.0 \mathrm{~Hz}$

$\begin{array}{rr}\text { POINT } & 32768 \\ \text { FREQU } & 27322.4\end{array}$

$\begin{array}{rr}\text { FREQU } & 27322.4 \\ \text { SCANS } & 2800\end{array}$

ACQTM $\quad 1.199 \mathrm{sec}$

PW1

IRNUC

SLVMT CDCL3

EXREF

$3.000 \mathrm{sec}$

6.3 u

CDCL3

$77.00 \mathrm{ppm}$

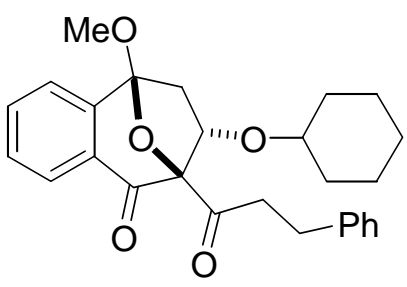




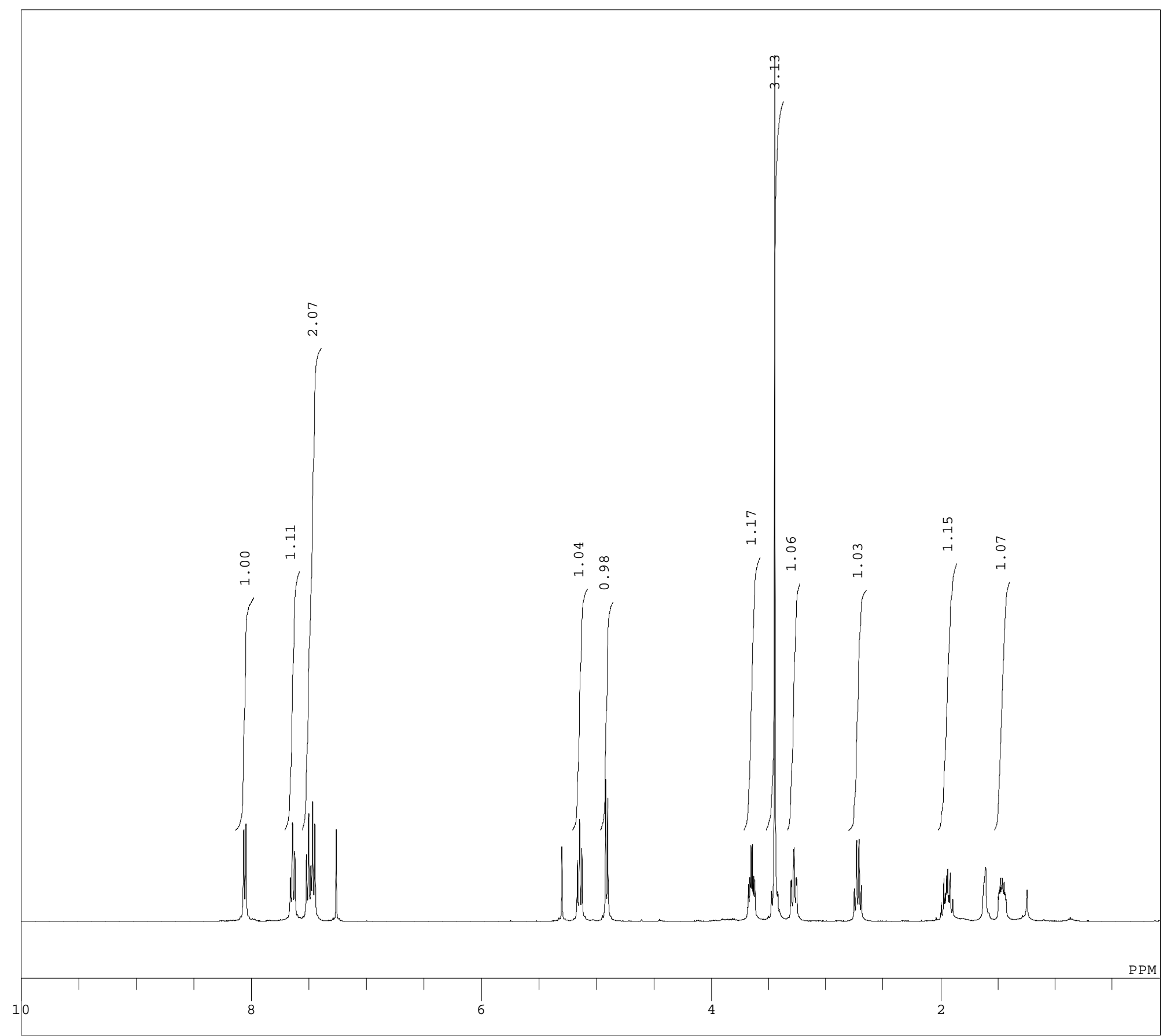

DFILE $\quad C: \backslash$ Documents and Settings $\backslash H$ Hro $\backslash f f f X f N f g f b f V$

COMNT DI

DATIM Fri Oct 27 12:39:31 200

OBNUC $1 \mathrm{H}$

EXMOD NON

OBFRQ $\quad 399.65 \mathrm{MHZ}$

OBSET $\quad 124.00 \mathrm{KHZ}$

OBFIN $\quad 10500.0 \mathrm{HZ}$

POINT 32768

FREQU $\quad 7993.6 \mathrm{~Hz}$

$\begin{array}{lr}\text { SCANS } & 8 \\ \text { ACOTM } & 4.099 \mathrm{sec}\end{array}$

$\begin{array}{ll}\text { ACQTM } & 4.099 \mathrm{sec} \\ \text { PD } & 4.950 \mathrm{sec}\end{array}$

PW1

$\begin{array}{lll}\text { IRNUC } & 1 \mathrm{H} & \\ \text { CTEMP } & & 20.5 \mathrm{C}\end{array}$

SLVNT CDCL3

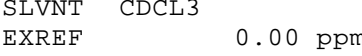

$.20 \mathrm{~Hz}$

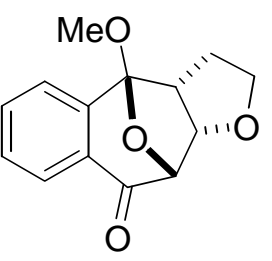




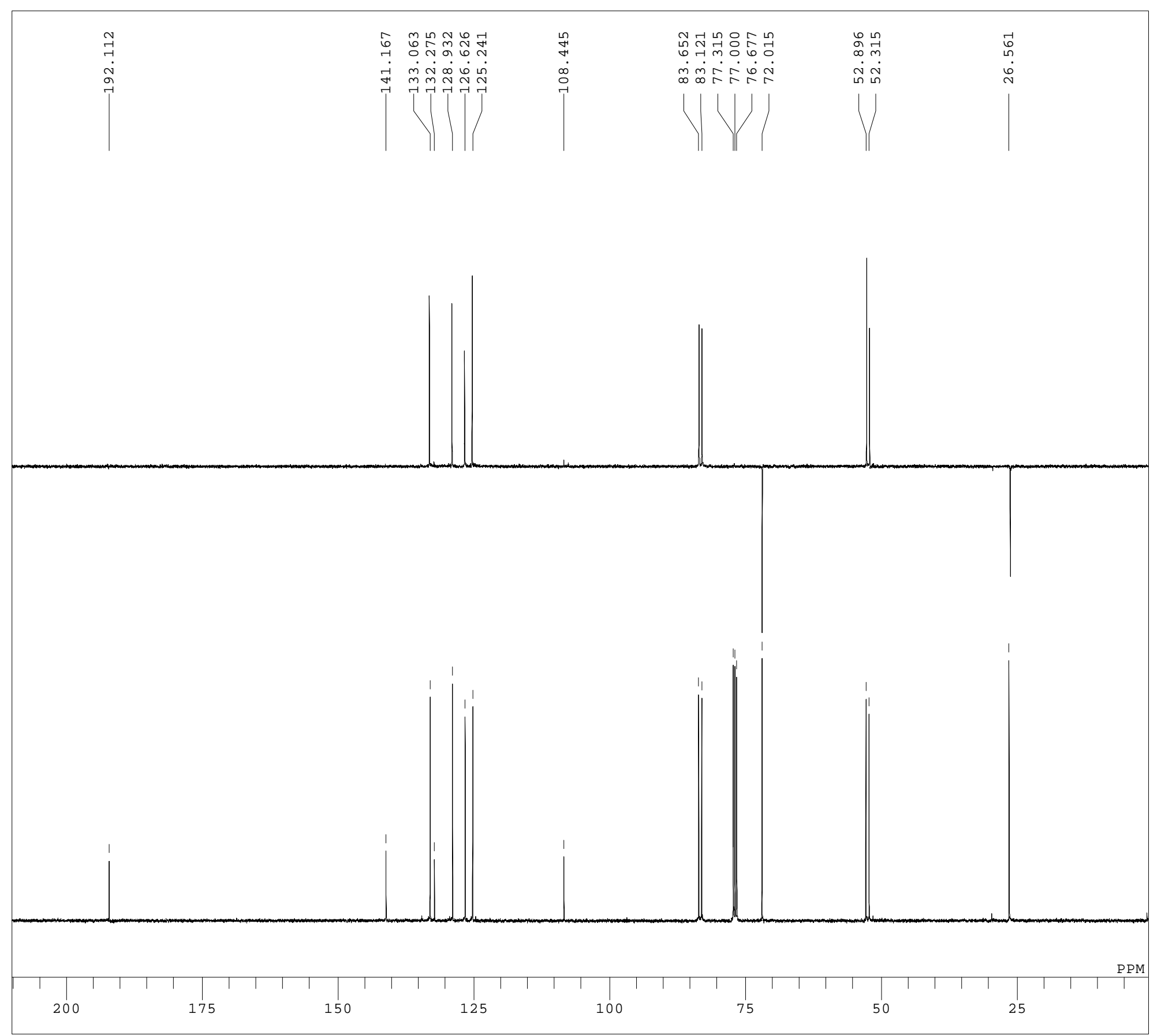

DFILE C: $\backslash$ Documents and Settings $\backslash H i r O \backslash f f f X f N f g f b f v$ COMNT DI-203-1DEPT

DATIM Tue Feb $20 \quad 04: 12: 20 \quad 200$

OBNUC $13 \mathrm{C}$

$\begin{array}{lll}\text { EXMOD } & \text { BCM } & \\ \text { OBFRQ } & & 100.40 \mathrm{MHZ}\end{array}$

$\begin{array}{ll}\text { OBFRQ } & 100.40 \mathrm{MHz} \\ \text { OBSET } & 125.00 \mathrm{KHz}\end{array}$

OBFIN $\quad 10500.0 \mathrm{~Hz}$

$\begin{array}{lr}\text { POINT } & 32768 \\ \text { FREQU } & 27322.4\end{array}$

SCANS 1800

ACQTM $\quad 1.199 \mathrm{sec}$

$\begin{array}{lr}\text { PD } & 3.000 \mathrm{sec} \\ \text { PW1 } & 6.3 \text { us }\end{array}$

IRNUC $1 \mathrm{H}$

CTEMP 25.5

SLVNT CDCL3

EXRE

$77.00 \mathrm{ppm}$

IN 31

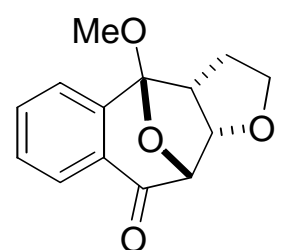




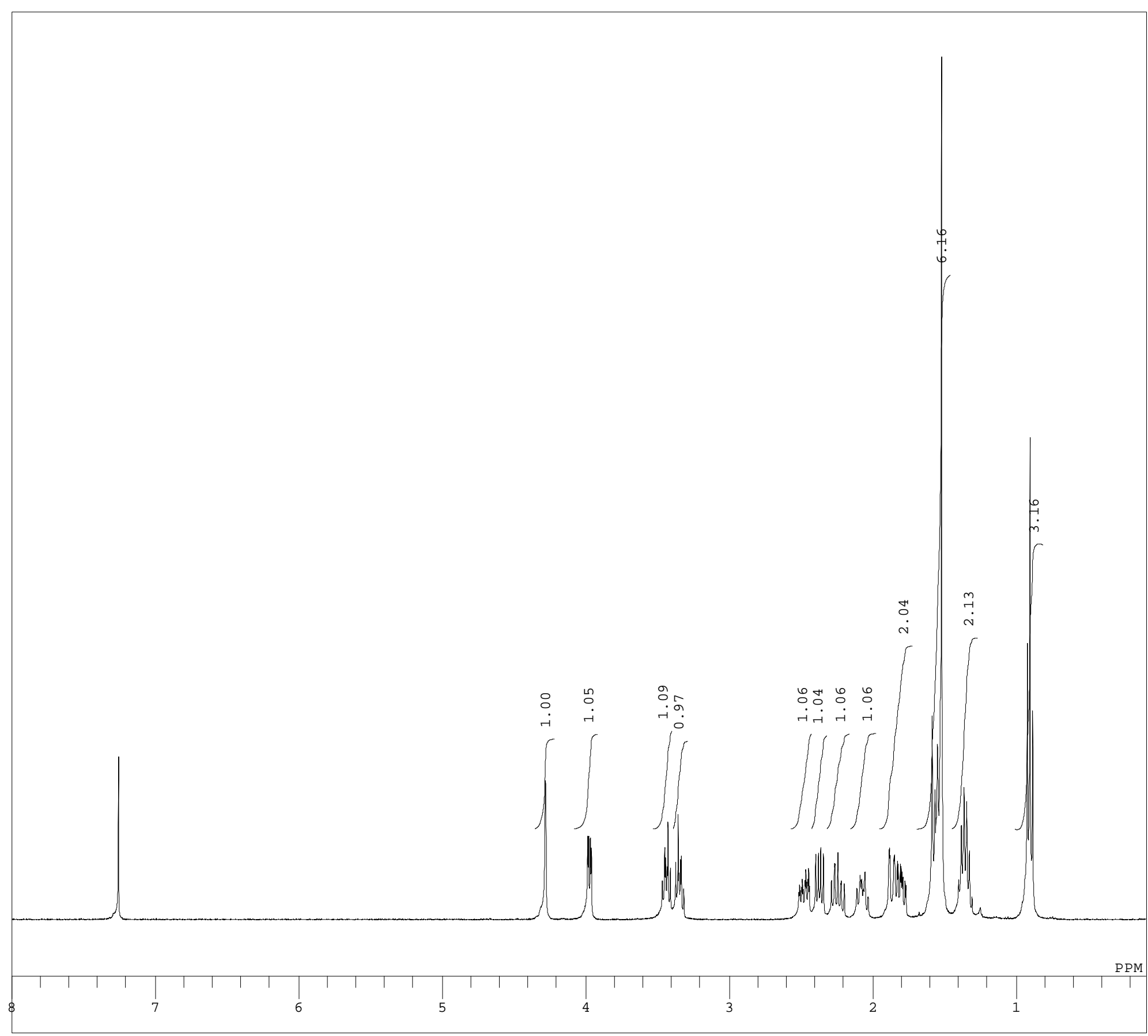

DFILE $\quad C: \backslash$ Documents and Settings $\backslash H i r o \backslash f f f X f N f g f b f v \backslash$ COMNT SH

DATIM Sun Mar 04 18:14:21 200

OBNUC $1 \mathrm{H}$

EXMOD NON

OBFRQ $\quad 399.65 \mathrm{MHZ}$

OBSET $\quad 124.00 \mathrm{KHZ}$

OBFIN $10500.0 \mathrm{HZ}$

POINT 32768

FREQU $\quad 7993.6 \mathrm{~Hz}$

$\begin{array}{lr}\text { SCANS } & 8 \\ \text { ACQTM } & 4.099 \mathrm{sec}\end{array}$

$\begin{array}{ll}\text { ACQTM } & 4.099 \mathrm{sec} \\ \mathrm{PD} & 4.950 \mathrm{sec}\end{array}$

PW1 10.0

IRNUC $1 \mathrm{H}$

SLVNT CDCL3

EXREF

$24.1 \mathrm{c}$

BF

$0.00 \mathrm{ppm}$

.24
18

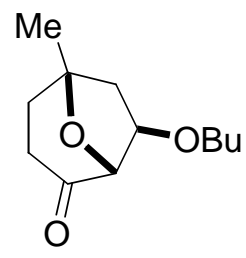




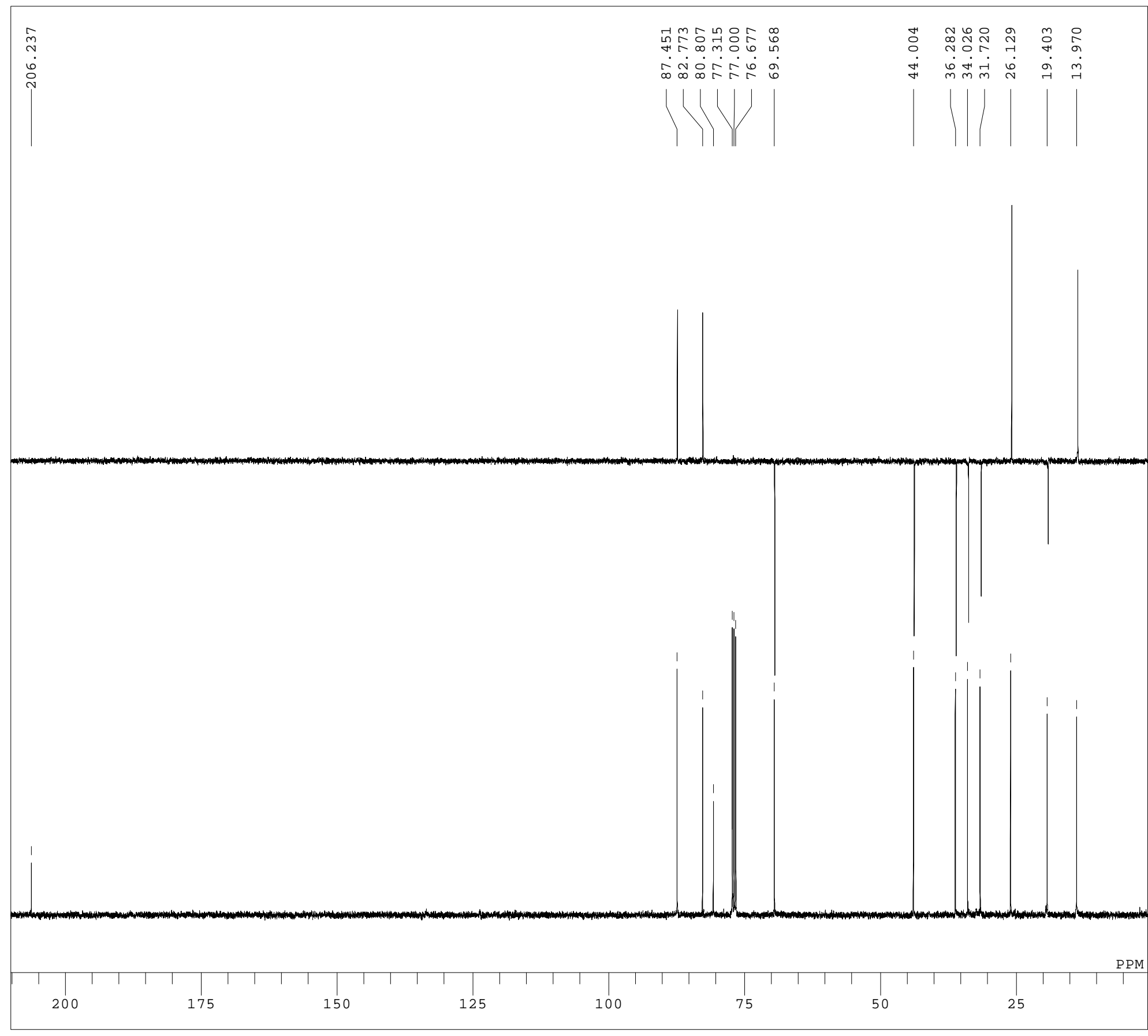

DFILE $\quad C: \backslash$ Documents and Settings $\backslash H i r o \backslash f f f X f N f g f b f v$ COMNT SH

DATIM Fri Jul 27 09:38:40 200

OBNUC 13C

EXMOD BCM

$\begin{array}{ll}\text { OBFRQ } & 100.40 \mathrm{MHz}\end{array}$

OBSET $\quad 125.00 \mathrm{KHZ}$

OBFIN $\quad 10500.0 \mathrm{~Hz}$

POINT 32768

FREQU 27322.4

SCANS 800

ACQTM $\quad 1.199 \mathrm{sec}$

$\begin{array}{lr}\text { PD } & 3.000 \mathrm{sec} \\ \text { PW1 } & 6.3 \text { us }\end{array}$

IRNUC $1 \mathrm{H}$

CTEMP CDCL 3

SLVNT

EXREF

$77.00 \mathrm{ppm}$

$1.20 \mathrm{~Hz}$

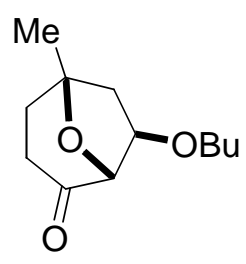




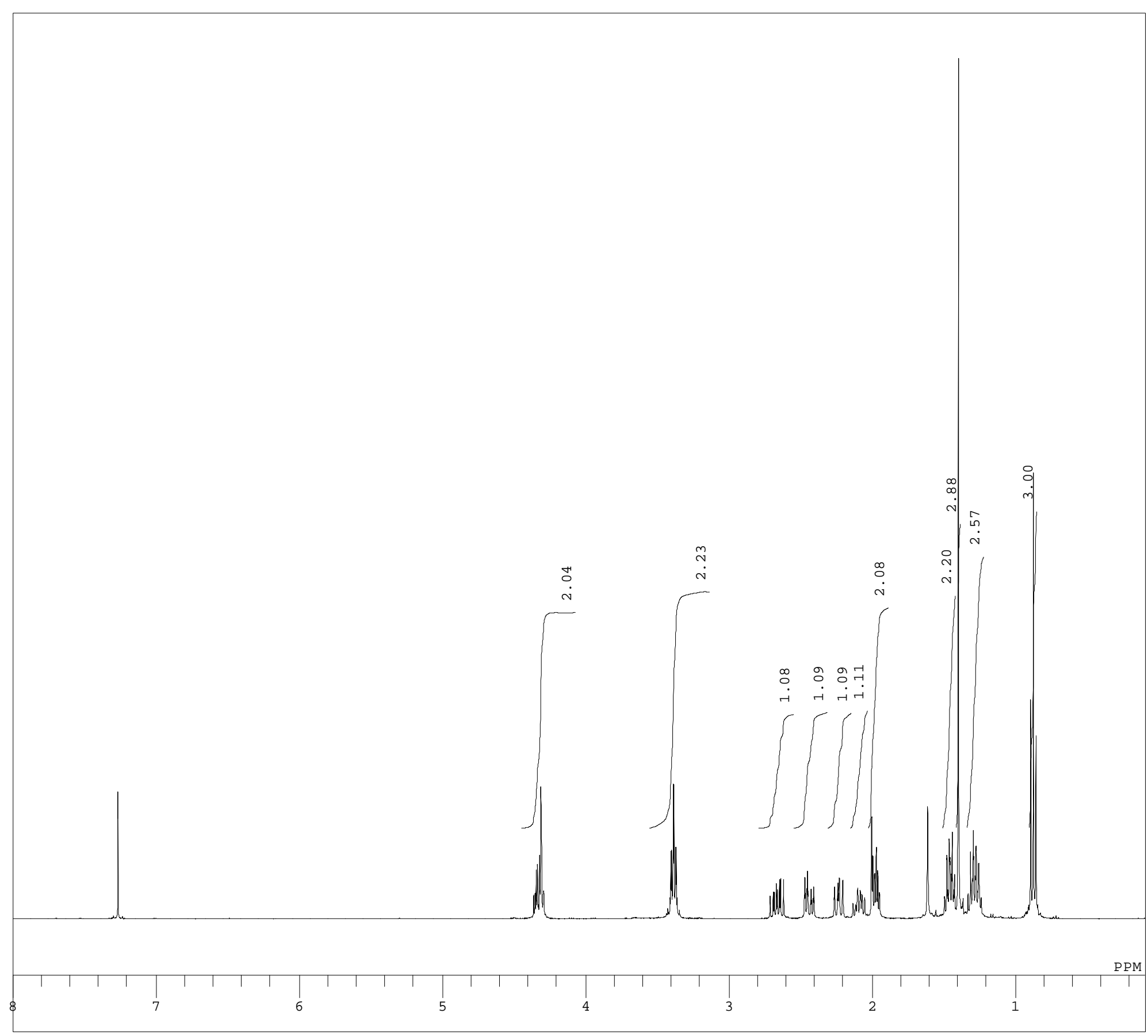

DFILE $\quad C: \backslash$ Documents and Settings $\backslash H$ iro $\backslash f f f X f N f g f b f V$ COMNT SH

DATIM Thu Nov 16 09:27:25 200

OBNUC $1 \mathrm{H}$

EXMOD NON

OBFRQ $\quad 399.65 \mathrm{MHZ}$

OBSET $\quad 124.00 \mathrm{KHZ}$

OBFIN $\quad 10500.0 \mathrm{HZ}$

POINT 32768

$\quad 8000.0 \mathrm{~Hz}$

$\begin{array}{lr}\text { SCANS } & 8 \\ \text { ACOTM } & 4.096 \mathrm{sec}\end{array}$

$\begin{array}{ll}\text { ACQTM } & 4.096 \mathrm{sec} \\ \text { PD } & 4.950 \mathrm{sec}\end{array}$

PW1 10.

IRNUC $\quad 1 \mathrm{H}$

SLVNT CDCL3

EXREF

$0.00 \mathrm{ppm}$ $.24 \mathrm{~Hz}$

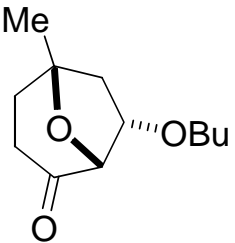

